Federal Reserve Bank of Minneapolis

Research Department Staff Report 408

August 2008

\title{
Liquidity in Asset Markets with Search Frictions*
}

\author{
Ricardo Lagos \\ New York University \\ Guillaume Rocheteau \\ Federal Reserve Bank of Cleveland
}

\begin{abstract}
We develop a search-theoretic model of financial intermediation and use it to study how trading frictions affect the distribution of asset holdings, asset prices, efficiency, and standard measures of liquidity. A distinctive feature of our theory is that it allows for unrestricted asset holdings, so market participants can accommodate trading frictions by adjusting their asset positions. We show that these individual responses of asset demands constitute a fundamental feature of illiquid markets: they are a key determinant of bid-ask spreads, trade volume, and trading delays - all the dimensions of market liquidity that search-based theories seek to explain.
\end{abstract}

*We are grateful to Gadi Barlevy, Darrell Duffie, Mariacristina De Nardi, Nicolae Gârleanu, Joe Haubrich, Rob Shimer, Neil Wallace, Pierre-Olivier Weill, and Ruilin Zhou for comments. We also thank seminar participants at the Bank of Portugal, Chicago GSB, Federal Reserve Bank of Cleveland, Federal Reserve Bank of New York, Indiana, HEC Lausanne, London Business School, Penn State, Princeton, Queens, Rice, Simon Fraser, Singapore Management University, Universidad Torcuato Di Tella, University of Basel, UCLA, UCSB, University of Pennsylvania, and University of Texas at Austin. We thank Patrick Higgins for research assistance. Financial support from the C. V. Starr Center for Applied Economics at NYU is gratefully acknowledged. The views expressed herein are those of the authors and not necessarily those of the Federal Reserve Bank of Cleveland, the Federal Reserve Bank of Minneapolis, or the Federal Reserve System. 


\section{Introduction}

A recent literature pioneered by Duffie, Gârleanu, and Pedersen (2005) (DGP hereafter) uses search theory to model the trading frictions characteristic of over-the-counter (OTC) markets. ${ }^{1}$ The search-based approach is appealing because it can parsimoniously rationalize standard measures of liquidity such as bid-ask spreads, execution delays, and trade volume, and can be used to study how market conditions influence these measures. A virtue of DGP's formulation is that it is analytically tractable, so all these mechanisms can be well understood.

The literature spurred by DGP, however, keeps the framework tractable by imposing a stark restriction on asset holdings, namely, that agents can only hold either 0 units or 1 unit of the asset. In effect, investors' ability to respond to changes in market conditions is limited rather severely by this restriction. In this paper we develop a search-based model of liquidity in asset markets with no restrictions on investors' asset holdings. The model is close in structure and spirit to DGP, but captures the heterogeneous responses of individual investors to changes in market conditions. From the broader perspective of search and matching theory, a striking feature of the model we develop is that it remains analytically tractable despite the large degree of heterogeneity among agents, which is propagated endogenously by random matching with unrestricted asset holdings. ${ }^{2}$ We provide a full characterization of the equilibrium, including transitional dynamics and the endogenous distribution of investors' asset positions.

Our methodological contribution provides new insights into how trading frictions affect outcomes in financial markets. We find that as a result of the restrictions they impose on asset holdings, existing search-based theories neglect a critical feature of illiquid markets, namely, the fact that market participants can mitigate trading frictions by adjusting their asset positions to reduce their trading needs. ${ }^{3}$ The key theoretical observation is that an investor's asset demand in an OTC market depends not only on his valuation for the asset at the time of the trade,

\footnotetext{
${ }^{1}$ The search-theoretic literature on financial markets also includes Duffie, Gârleanu, and Pedersen (2007), Gârleanu (2006), Miao (2006), Rust and Hall (2003), Spulber (1996), and Weill (2007).

${ }^{2}$ DGP restricted asset holdings for the same technical reasons why Kiyotaki and Wright (1993) restricted money holdings to $\{0,1\}$, i.e., to keep the endogenous distribution of asset holdings manageable. Aside from a few exceptions, such as Green and Zhou (2002), the recent monetary literature, e.g., Lagos and Wright (2005), allows for unrestricted portfolios and keeps the analysis tractable by making assumptions that render the equilibrium distribution of money holdings degenerate. By way of comparison, the model we develop here allows for unrestricted asset holdings and remains tractable, even though we make no attempt to harness the heterogeneity that is generated by the model dynamics.

${ }^{3}$ The importance of this mechanism in the context of another class of models - those with exogenous transaction costs - has been stressed by Constantinides (1986) for the case of proportional transaction costs, and by Lo, Mamaysky, and Wang (2004) for the case of fixed transaction costs.
} 
but also on his expected valuation over the holding period until his next opportunity to trade. A reduction in trading delays makes investors less likely to remain locked into an undesirable asset position and therefore induces them to put more weight on their current valuation. As a result, reductions in trading frictions induce an investor to demand a larger asset position if his current valuation is relatively high, and a smaller position if it is relatively low. We find that these responses of individual asset demands are a key determinant of market efficiency and asset prices as well as bid-ask spreads, trade volume, and trading delays - precisely the dimensions of market liquidity that search-based theories of financial intermediation are designed to explain.

From an investor's standpoint, bid-ask spreads constitute the main out-of-pocket transaction cost in an illiquid market. We show that spreads depend not only on the ease with which investors can find alternative trading partners (a mechanism identified in the existing literature), but also on the degree of mismatch between investors' endogenous asset positions and their current valuations of the asset. Our model predicts a distribution of transaction costs, both across trade sizes - with spreads that increase with the size of the trade - as well as within a given trade-size category - across investors with different valuations.

Trade volume is a manifestation of the ability of the exchange mechanism to reallocate assets across investors. We find that a reduction in trading delays shifts the equilibrium distribution of asset holdings in a way that tends to increase trade volume. Our theory reveals that from an investor's point of view, an increase in the market power of dealers is isomorphic to an increase in trading delays. Hence, trade volume will tend to be small in markets where dealers enjoy a large degree of market power. These effects are implicitly assumed away if asset holdings are restricted to lie in $\{0,1\}$.

Finally, we allow for free entry of dealers as a way to endogenize trading delays - a distinguishing feature of the microstructure of an OTC market. We find that when interacted with investors' unrestricted asset holding decisions, the dealers' incentives to make markets generate a liquidity externality that can give rise to multiple steady states. This finding suggests that all the symptoms of an illiquid market-large spreads, small trade volume, and long trading delays - can simultaneously arise as a self-fulfilling phenomenon in asset markets with an OTC structure. 


\section{Environment}

Time is continuous, starts at $t=0$, and goes on forever. There are two types of infinitely lived agents: a unit measure of investors and a unit measure of dealers. There is one asset, one perishable consumption good called fruit, and another consumption good defined as numéraire. The asset is durable, perfectly divisible, and in fixed supply, $A \in \mathbb{R}_{+}$. Each unit of the asset produces a unit flow of fruit. There is no market for fruit, so holding the asset is necessary to consume this good. The numéraire good is produced and consumed by all agents. The instantaneous utility function of an investor is $u_{i}(a)+c$, where $a \in \mathbb{R}_{+}$represents the fruit consumption (which coincides with the investor's asset holdings), $c \in \mathbb{R}$ is the net consumption of the numéraire good ( $c<0$ if the investor produces more of these goods than he consumes), and $i \in \mathbb{X}=\{1, \ldots, I\}$ indexes a preference type. The utility function $u_{i}(a)$ is twice continuously differentiable, strictly increasing, and strictly concave. ${ }^{4}$ Each investor receives a preference shock with Poisson arrival rate $\delta$. This process is independent across investors. Conditional on the preference shock, the probability the investor draws preference type $i$ is $\pi_{i}>0$, with $\sum_{i=1}^{I} \pi_{i}=1$. These preference shocks capture the notion that investors will value the asset differently over time, thereby generating the need to rebalance their asset positions. ${ }^{5}$ Dealers do not hold positions and their instantaneous utility is $c$, their consumption of the numéraire good. ${ }^{6}$ All agents discount at rate $r>0$.

Dealers can trade the asset continuously in a competitive interdealer market. Investors periodically contact dealers who can trade in this market on their behalf. Meetings with dealers occur at random according to a Poisson process with arrival rate $\alpha .^{7}$ Once a dealer and an

\footnotetext{
${ }^{4}$ Just as in DGP, our specification associates a certain utility to the investor as a function of his asset holdings. The utility from holding an asset position could be simply the value from enjoying the asset itself, as would be the case for real assets such as cars or houses. An alternative interpretation that leads to the same formulation would be to assume that there is a single consumption good, that investors are risk-neutral and able to borrow and lend freely at rate $r$, and regard the asset as physical capital used to produce the consumption good with the production technology $u_{i}$. As yet another possibility, one could adopt the preferred interpretation of DGP, namely that $u_{i}$ is in fact a reduced-form utility function that stands in for the various reasons why investors may want to hold different quantities of the asset, such as differences in liquidity needs, financing or financial-distress costs, correlation of asset returns with endowments (hedging needs), or relative tax disadvantages. By now, several papers that build on the work of DGP have formalized the "hedging needs" interpretation. Examples include Duffie, Gârleanu, and Pedersen (2007), Gârleanu (2006), and Vayanos and Weill (2008).

${ }^{5}$ In Appendix B, we allow preference shocks to follow a general continuous-time Markov chain and find that most of the substantive results generalize under appropriate regularity conditions.

${ }^{6}$ The restriction that dealers cannot hold assets is immaterial when analyzing steady-state equilibria. Lagos, Rocheteau, and Weill (2007) study dynamic equilibria where dealers may choose to hold asset positions.

${ }^{7}$ Although our description of the trading process is stylized, it captures the salient features of the actual trading arrangements in OTC markets. We refer the interested reader to Schultz (2001) as well as the discussion
} 
investor have contacted each other, they negotiate over the quantity of assets that the dealer will acquire for the investor and the intermediation fee that the dealer charges for his services. After the transaction has been completed, the dealer and the investor part ways.

Asset holdings and preference types lie in the sets $\mathbb{R}_{+}$and $\mathbb{X}$, respectively, and vary across investors and over time. We describe this heterogeneity with a probability space $\left(\mathbb{S}, \Sigma, H_{t}\right)$, where $\mathbb{S}=\mathbb{R}_{+} \times \mathbb{X}, \Sigma$ is the $\sigma$-field generated by the sets $(\mathcal{A}, \mathcal{I})$, where $\mathcal{A} \subseteq \mathbb{R}_{+}$and $\mathcal{I} \subseteq \mathbb{X}$, and $H_{t}$ is a probability measure on $\Sigma$ that represents the distribution of investors across asset holdings and preference types at time $t$.

\section{Equilibrium}

Let $V_{i}(a, t)$ denote the maximum expected discounted utility attainable by an investor who has preference type $i$ and is holding $a$ assets at time $t$. The value function $V_{i}(a, t)$ satisfies

$$
\begin{aligned}
V_{i}(a, t) & =\mathbb{E}_{i}\left[\int_{t}^{T_{\alpha}} e^{-r(s-t)} u_{k(s)}(a) d s\right. \\
& \left.+e^{-r\left(T_{\alpha}-t\right)}\left\{V_{k\left(T_{\alpha}\right)}\left[a_{k\left(T_{\alpha}\right)}\left(T_{\alpha}\right), T_{\alpha}\right]-p\left(T_{\alpha}\right)\left[a_{k\left(T_{\alpha}\right)}\left(T_{\alpha}\right)-a\right]-\phi_{k\left(T_{\alpha}\right)}\left(a, T_{\alpha}\right)\right\}\right],
\end{aligned}
$$

where $T_{\alpha}$ denotes the next time the investor contacts a dealer and $k(s) \in \mathbb{X}$ the investor's preference type at time $s$. The expectations operator, $\mathbb{E}_{i}$, is over the random variables $T_{\alpha}$ and $k(s)$ and is indexed by $i$ to indicate that it is conditional on $k(t)=i$. The first term on the right side of (1) contains the expected discounted utility flows over the time interval $\left[t, T_{\alpha}\right]$, whose length is exponentially distributed with mean $1 / \alpha$. The flow utility is indexed by the preference type, $k(s)$, which follows a compound Poisson process. The second term on the right side of (1) is the expected discounted utility from the time when the investor next contacts a dealer, $T_{\alpha}$, onward. At this time $T_{\alpha}$, the dealer purchases $a_{k\left(T_{\alpha}\right)}\left(T_{\alpha}\right)-a$ in the market (or sells if this quantity is negative) at price $p\left(T_{\alpha}\right)$ on behalf of the investor; the investor readjusts his asset holdings from $a$ to $a_{k\left(T_{\alpha}\right)}\left(T_{\alpha}\right)$ and pays the dealer an intermediation fee $\phi_{k\left(T_{\alpha}\right)}\left(a, T_{\alpha}\right)$. Both the fee and the asset price are expressed in terms of the numéraire good. ${ }^{8}$

in Section 2.1 in Lagos and Rocheteau (2006).

${ }^{8}$ Since the intermediation fee is determined in a bilateral meeting, it may depend on the investor's preference type and asset holdings. Our notation for the investor's new asset position, $a_{k\left(T_{\alpha}\right)}\left(T_{\alpha}\right)$, makes explicit that it may depend on time and on the investor's preference type at the time of the trade. Below (condition (3)), we will find that the investor's new asset position is independent of the asset position he was holding at the time of the trade. To simplify the notation we anticipate this result and do not include $a$ as an argument of his new asset position. 
Let $W(t)$ denote the maximum expected discounted utility attainable by a dealer. It satisfies

$$
W(t)=\mathbb{E}\left\{e^{-r\left(T_{\alpha}-t\right)}\left[\int_{\mathbb{S}} \phi_{i}\left(a, T_{\alpha}\right) d H_{T_{\alpha}}+W\left(T_{\alpha}\right)\right]\right\}
$$

where the expectations operator, $\mathbb{E}$, is over the next time the dealer meets an investor, $T_{\alpha}$. Random matching implies that the investor whom the dealer meets is a random draw from $H_{T_{\alpha}}$, the distribution of investors across preference types and asset holdings at time $T_{\alpha}$.

We turn to the determination of the terms of trade in a bilateral meeting at time $t$ between a dealer and an investor of type $i$ who is holding $a$. Let $a^{\prime}$ denote the investor's post-trade asset holdings and $\phi$ the intermediation fee. We take $\left(a^{\prime}, \phi\right)$ to be the outcome corresponding to the Nash solution to a bargaining problem where the dealer has bargaining power $\eta \in[0,1]$. The utility of the investor is $V_{i}\left(a^{\prime}, t\right)-p(t)\left(a^{\prime}-a\right)-\phi$ if an agreement $\left(a^{\prime}, \phi\right)$ is reached, and $V_{i}(a, t)$ in case of disagreement. Therefore, the investor's gain from trade is $V_{i}\left(a^{\prime}, t\right)-V_{i}(a, t)-$ $p(t)\left(a^{\prime}-a\right)-\phi$. Analogously, the utility of the dealer is $W(t)+\phi$ if an agreement $\left(a^{\prime}, \phi\right)$ is reached and $W(t)$ in case of disagreement, so the dealer's gain from trade is the fee, $\phi$. The bargaining outcome is

$$
\left[a_{i}(t), \phi_{i}(a, t)\right]=\arg \max _{\left(a^{\prime}, \phi\right)}\left[V_{i}\left(a^{\prime}, t\right)-V_{i}(a, t)-p(t)\left(a^{\prime}-a\right)-\phi\right]^{1-\eta} \phi^{\eta}
$$

where the maximization is subject to $a^{\prime} \geq 0 .{ }^{9}$ The solution (2) can be written as

$$
\begin{aligned}
a_{i}(t) & =\arg \max _{a^{\prime} \geq 0}\left[V_{i}\left(a^{\prime}, t\right)-p(t) a^{\prime}\right] \\
\phi_{i}(a, t) & =\eta\left\{V_{i}\left[a_{i}(t), t\right]-V_{i}(a, t)-p(t)\left[a_{i}(t)-a\right]\right\} .
\end{aligned}
$$

We now turn to the investor's problem. Substitute (3) and (4) into (1) to obtain

$$
\begin{aligned}
V_{i}(a, t) & =\mathbb{E}_{i}\left[\int_{t}^{T_{\alpha}} e^{-r(s-t)} u_{k(s)}(a) d s\right. \\
& \left.+e^{-r\left(T_{\alpha}-t\right)}\left\{(1-\eta) \max _{a^{\prime} \geq 0}\left[V_{k\left(T_{\alpha}\right)}\left(a^{\prime}, T_{\alpha}\right)-p\left(T_{\alpha}\right)\left(a^{\prime}-a\right)\right]+\eta V_{k\left(T_{\alpha}\right)}\left(a, T_{\alpha}\right)\right\}\right] .
\end{aligned}
$$

It is apparent from (5) that the investor's payoff is the same he would get in an alternative environment where he meets dealers according to a Poisson process with arrival rate $\alpha$, but

\footnotetext{
${ }^{9}$ It would be equivalent to set $\phi=(\hat{p}-p(t))\left(a^{\prime}-a\right)$ in $(2)$ and reformulate the bargaining problem as a choice of $\left(a^{\prime}-a, \hat{p}\right)$. If $a^{\prime}>a$ the investor is a buyer and $\hat{p}>p(t)$ can be interpreted as the ask price he is charged by the dealer. Conversely, if $a^{\prime}<a$ the investor is a seller and $\hat{p}<p(t)$ is the bid price he is paid by the dealer. In Appendix $\mathrm{C}$, we formulate several strategic bargaining games, each with a unique subgame perfect equilibrium outcome that coincides with the axiomatic Nash solution we have adopted here.
} 
instead of bargaining, he readjusts his asset position and extracts the whole surplus with probability $1-\eta$, whereas with probability $\eta$ he cannot readjust his asset position and enjoys no gain from trade. Therefore, from the standpoint of the investor, keeping the paths of the aggregate variables unchanged, the environment we are analyzing is payoff-equivalent to an alternative one in which he meets dealers according to a Poisson process with arrival rate $\kappa=\alpha(1-\eta)$ and has all the bargaining power in bilateral negotiations. Based on this observation, the following lemma offers an equivalent formulation of the investor's choice of asset holdings that appears on the right side of (5).

Lemma 1 An investor with preference type $i$ and asset holdings a who readjusts his asset position at time $t$ solves

$$
\max _{a^{\prime} \geq 0}\left[\bar{u}_{i}\left(a^{\prime}\right)-q(t) a^{\prime}\right]
$$

where

$$
\begin{aligned}
\bar{u}_{i}(a) & =\frac{(r+\kappa) u_{i}(a)+\delta \sum_{j} \pi_{j} u_{j}(a)}{r+\kappa+\delta} \\
q(t) & =(r+\kappa)\left[p(t)-\kappa \int_{0}^{\infty} e^{-(r+\kappa) s} p(t+s) d s\right] .
\end{aligned}
$$

In Lemma $1, \bar{u}_{i}(a) /(r+\kappa)$ is the expected discounted utility and $q(t) /(r+\kappa)=p(t)-$ $\mathbb{E}\left[e^{-r\left(T_{\kappa}-t\right)} p\left(T_{\kappa}\right)\right]$ the present value of the expected capital loss to the investor from holding $a$ from $t$ until the next (effective) time $T_{\kappa}$ when he readjusts his holdings, where $T_{\kappa}-t$ is exponentially distributed with mean $1 / \kappa$. A choice of asset holdings, $a_{i}(t)$, must satisfy

$$
\bar{u}_{i}^{\prime}\left[a_{i}(t)\right] \leq q(t), \quad \text { "= } \quad \text { if } a_{i}(t)>0 .
$$

Given $q(t)$, the following lemma shows how to recover $p(t) .{ }^{10}$

Lemma 2 If $\lim _{t \rightarrow \infty} e^{-r t} p(t)=0$, the price of the asset is

$$
p(t)=\int_{t}^{\infty} e^{-r(s-t)}\left[q(s)-\frac{\dot{q}(s)}{r+\kappa}\right] d s .
$$

At this point we can simplify the expression for the intermediation fee. From $(4), \phi_{i}(a, t)=$ $\eta\left\{V_{i}\left[a_{i}(t), t\right]-V_{i}(a, t)-p(t)\left[a_{i}(t)-a\right]\right\}$, with $a_{i}(t)$ characterized by (9). If we substitute the value functions (e.g., (27) from the appendix), we arrive at

$$
\phi_{i}(a, t)=\frac{\eta\left\{\bar{u}_{i}\left[a_{i}(t)\right]-\bar{u}_{i}(a)-q(t)\left[a_{i}(t)-a\right]\right\}}{r+\kappa} .
$$

\footnotetext{
${ }^{10}$ In Appendix D, we show that $p(t)$ must satisfy $\lim _{t \rightarrow \infty} e^{-r t} p(t)=0$ in any equilibrium, so we can appeal to this condition without loss of generality.
} 
Since each investor contacts a dealer with equal probability, the quantity of assets supplied in the interdealer market over a small interval of time $d t$ is $\alpha d t A .{ }^{11}$ Similarly, the measure of type- $i$ investors who contact dealers is $\alpha d t n_{i}(t)$, where

$$
n_{i}(t)=e^{-\delta t} n_{i}(0)+\left(1-e^{-\delta t}\right) \pi_{i}
$$

is the measure of investors with preference type $i$ at time $t$, so the demand for assets in the interdealer market is $\alpha d t \sum_{i=1}^{I} n_{i}(t) a_{i}(t)$. The clearing condition for the asset market is

$$
\sum_{i=1}^{I} n_{i}(t) a_{i}(t)=A
$$

At any point in time, investors differ in asset holdings and preference types. Consider a set of asset holdings $\mathcal{A}$ and a set of preference types $\mathcal{I}$, then for all $(\mathcal{A}, \mathcal{I}) \in \Sigma, H_{t}(\mathcal{A}, \mathcal{I})$ gives the measure of investors whose asset holdings and preference types lie in $\mathcal{A}$ and $\mathcal{I}$, respectively. We characterize this probability measure in the following lemma, where $\mathbb{I}_{\{a \in \mathcal{A}\}}$ denotes an indicator function that equals 1 if $a \in \mathcal{A}$.

Lemma 3 The measure of investors across individual states at time $t$ satisfies

$$
H_{t}(\mathcal{A}, \mathcal{I})=\sum_{i \in \mathcal{I}} \sum_{j=1}^{I}\left[n_{j i}^{0}(\mathcal{A}, t)+\int_{0}^{t} \mathbb{I}_{\left\{a_{j}(t-\tau) \in \mathcal{A}\right\}} n_{j i}(\tau, t) d \tau\right]
$$

for all $(\mathcal{A}, \mathcal{I}) \in \Sigma$, where

$$
n_{j i}^{0}(\mathcal{A}, t)=e^{-\alpha t}\left[\left(1-e^{-\delta t}\right) \pi_{i}+e^{-\delta t} \mathbb{I}_{\{i=j\}}\right] H_{0}(\mathcal{A},\{j\})
$$

and

$$
n_{j i}(\tau, t)=\alpha e^{-\alpha \tau}\left[\left(1-e^{-\delta \tau}\right) \pi_{i}+e^{-\delta \tau} \mathbb{I}_{\{i=j\}}\right] n_{j}(t-\tau)
$$

At time 0, the market starts with investors distributed across preference types and asset holdings according to the initial probability measure $H_{0}$. Subsequently, there are two types of investors: those who have not contacted a dealer since time 0 and those who have. The time- $t$ measure of those who started at time 0 with preference type $j$ and assets in $\mathcal{A}$, whose preference type is $i$ at the current time $t$, and who have never traded (so their asset holdings are still in $\mathcal{A}$ ) is $n_{j i}^{0}(\mathcal{A}, t)$ as given in (15). Analogously, $n_{j i}(\tau, t)$ in (16) gives the time- $t$ density of investors

\footnotetext{
${ }^{11}$ See Duffie and Sun (2007) for a derivation of the law of large numbers in random-matching environments.
} 
whose last trade was at time $t-\tau$ when their preference type was $j$ and who have preference type $i$ at time $t$.

We are now ready to define equilibrium.

Definition 1 An equilibrium is a time-path $\left\langle\left\{a_{i}(t)\right\}, q(t), p(t),\left\{\phi_{i}(a, t)\right\}, H_{t}\right\rangle$ that satisfies (9), (10), (11), (13), and (14), given an initial condition $H_{0}$.

Proposition 1 There exists a unique equilibrium.

To illustrate how a reduction in trading delays affects the equilibrium, consider the limiting case $\alpha \rightarrow \infty$. From (7), $\bar{u}_{i}(a) \rightarrow u_{i}(a)$ and from (8) and (9), $u_{i}^{\prime}\left[a_{i}(t)\right] \leq q(t)=r p(t)-\dot{p}(t)$ for all $i$. From $(13), q(t) \rightarrow q^{*}(t)$, which solves $\sum_{i \in \mathcal{I}_{t}^{+}} n_{i}(t) u_{i}^{\prime-1}\left[q^{*}(t)\right]=A$, where $\mathcal{I}_{t}^{+}=$ $\left\{i \in \mathbb{X}: a_{i}(t)>0\right\}$. From $(11), \phi_{i}(a, t) \rightarrow 0$ for all $a, i$ and $t$. With regard to the distribution of investors, $\alpha \rightarrow \infty$ implies that every investor holds his desired asset position at all times. ${ }^{12}$ Thus, as frictions vanish, investors choose $a_{i}(t)$ continuously by equating their current marginal utility from holding the asset to its effective cost $q^{*}(t)$, and the equilibrium fees, asset price, and distribution of asset holdings are the ones that would prevail in a Walrasian economy.

\subsection{Efficiency}

Consider a social planner who wishes to maximize the sum of all agents' expected discounted utilities, subject to the same meeting frictions that agents face in the decentralized formulation. Specifically, over a small time interval of length $d t$, the planner can only reallocate assets among a measure $\alpha d t$ of investors chosen at random from the population.

Since the utility of consumption and the disutility of production of the numéraire good net out to zero across agents, the planner solves

$$
\begin{gathered}
\max _{\left\{a_{i}(t)\right\}_{i=1}^{I}}\left\{K_{0}+\int_{0}^{\infty} \sum_{i=1}^{I} e^{-r t} \alpha n_{i}(t) \hat{U}_{i}\left[a_{i}(t)\right] d t\right\} \\
\text { s.t. } \sum_{i=1}^{I} \alpha n_{i}(t) a_{i}(t) \leq \alpha A,
\end{gathered}
$$

\footnotetext{
${ }^{12}$ To see this, first note that (15) implies the measure of agents who have not contacted a dealer since time 0 vanishes; i.e., $n_{j i}^{0}(\mathcal{A}, t) \rightarrow 0$ for all $i$ and $j$, all $t$ and all $\mathcal{A} \subseteq \mathbb{R}_{+}$as $\alpha \rightarrow \infty$. The time- $t$ density of agents who have not contacted a dealer since time $t-\tau>0$ is $n(\tau, t)=\sum_{i, j=1}^{I} n_{j i}(\tau, t)$. From $(16), \alpha \rightarrow \infty$ implies $n(\tau, t) \rightarrow 0$ for all $\tau>0$, i.e., investors can find a dealer instantly when $\alpha$ is arbitrarily large, so the measure of investors who have not met a dealer between $t-\tau$ and $t$ is zero for all $\tau>0$. As for those investors who have met a dealer this "instant," from $(16), n_{j i}(0, t)=0$ for $i \neq j$ and $n_{i i}(0, t)=n_{i}(t)$. Therefore, $H_{t}(\mathcal{A}, \mathcal{I}) \rightarrow \sum_{i \in \mathcal{I}} \mathbb{I}_{\left\{a_{i}(t) \in \mathcal{A}\right\}} n_{i}(t)$ as $\alpha \rightarrow \infty$, i.e., every investor of type $i$ holds $a_{i}(t)$ at every $t$.
} 
(12), and $a_{i}(t) \geq 0$ for $i \in \mathbb{X}$, where

$$
\hat{U}_{i}\left[a_{i}(t)\right]=\mathbb{E}_{i}\left[\int_{t}^{T_{\alpha}} e^{-r(s-t)} u_{k(s)}\left[a_{i}(t)\right] d s\right],
$$

$K_{0} \equiv \int_{\mathbb{S}} \hat{U}_{i}(a) d H_{0}$, and $H_{0}$ is given. The constant $K_{0}$ captures the utility of all investors before they trade for the first time. The second term in the objective states that over a time interval of length $d t$, there is a measure $\alpha n_{i}(t) d t$ of investors of type $i$ whose asset holdings can be rebalanced.

Proposition 2 The equilibrium is efficient if and only if $\eta=0$.

When an investor conducts a trade, he anticipates that the fee he will be charged to rebalance his asset holdings in his next encounter with a dealer will be increasing in the gains from that future trade. Hence, $\eta>0$ inefficiently discourages investors from taking positions that tend to lead to large asset reallocations in the future.

\subsection{Steady state}

Next, we characterize the limit of the equilibrium allocations and prices as $t \rightarrow \infty$. $^{13}$

Proposition 3 For any $H_{0}$, the equilibrium allocations and prices described in Definition $1,\left\langle\left\{a_{i}(t)\right\}, q(t), p(t),\left\{\phi_{i}(a, t)\right\}, H_{t}\right\rangle$, converge to the unique steady-state allocations and prices $\left\langle\left\{a_{i}\right\}, q, p,\left\{\phi_{i}(a)\right\}, H\right\rangle$, that satisfy $p=q / r$,

$$
\begin{aligned}
\bar{u}_{i}^{\prime}\left(a_{i}\right) & \leq q \quad \text { " }=" \quad \text { if } a_{i}>0, \\
\sum_{i=1}^{I} \pi_{i} a_{i} & =A, \\
\phi_{i}(a) & =\frac{\eta\left[\bar{u}_{i}\left(a_{i}\right)-\bar{u}_{i}(a)-q\left(a_{i}-a\right)\right]}{r+\kappa} \\
H\left(\left\{a_{i}\right\},\{j\}\right) & =\frac{\delta \pi_{i} \pi_{j}+\alpha \pi_{i} \mathbb{I}_{\{i=j\}}}{\alpha+\delta}
\end{aligned}
$$

and $H(\mathcal{A}, \mathcal{I})=0$ for all $(\mathcal{A}, \mathcal{I}) \in \Sigma$ such that $\bigcup_{j=1}^{I}\left\{a_{j}\right\} \cap \mathcal{A}=\varnothing$.

In what follows, when we analyze the steady state we will denote an individual investor's state $\left(a_{i}, j\right) \in\left\{a_{i}\right\}_{i=1}^{I} \times \mathbb{X}$ by $(i, j) \in \mathbb{X}^{2}$ and $H\left(\left\{a_{i}\right\},\{j\}\right)$ by $n_{i j}$. Also, at times we use $\phi_{j i}$ to denote $\phi_{i}\left(a_{j}\right)$ for $(i, j) \in \mathbb{X}^{2}$.

\footnotetext{
${ }^{13}$ We omit the " $t$ " argument in an endogenous variable when we refer to its steady-state value.
} 


\section{Asset holdings, prices, and trade volume}

In this section we focus on the steady state to study the effects of trading frictions on individual asset holdings, asset prices, and trade volume. Hereafter we assume $u_{i}^{\prime}(\infty)=0$ and $u_{i}^{\prime}(0)=\infty$ for each $i{ }^{14}$ Condition (18) becomes

$$
\bar{u}_{i}^{\prime}\left(a_{i}\right)=r p .
$$

Let $a_{i}=g_{i}(\kappa ; p)$ denote the choice of asset holdings characterized by $(22)$. Then

$$
\frac{\partial g_{i}(\kappa ; p)}{\partial \kappa}=\frac{\delta\left[u_{i}^{\prime}\left(a_{i}\right)-\sum_{j=1}^{I} \pi_{j} u_{j}^{\prime}\left(a_{i}\right)\right]}{-\bar{u}_{i}^{\prime \prime}\left(a_{i}\right)(r+\kappa+\delta)^{2}}
$$

has the sign of $u_{i}^{\prime}\left(a_{i}\right)-\sum_{j=1}^{I} \pi_{j} u_{j}^{\prime}\left(a_{i}\right)$, i.e., an investor whose current marginal valuation exceeds his expected marginal valuation over the expected holding period increases his demand when $\kappa$ increases. If $u_{i}^{\prime}\left(a_{i}\right)>\sum_{j=1}^{I} \pi_{j} u_{j}^{\prime}\left(a_{i}\right)$, the investor anticipates that his valuation is likely to revert toward $\sum_{j=1}^{I} \pi_{j} u_{j}^{\prime}\left(a_{i}\right)$ in the future, and that when this happens, he may be unable to rebalance his asset position for some time. Consequently, from (22), his choice of $a_{i}$ is lower than $u_{i}^{\prime-1}(r p)$, what he would choose in a world with no trading delays. If $\alpha$ increases, the investor is more likely to find a dealer faster; if $\eta$ decreases, it will be cheaper for the investor to readjust his asset holdings once he finds a dealer. In both cases, the investor assigns more weight to current marginal utility from holding the asset relative to the expected value, so his demand increases. Conversely, an investor with a current marginal valuation that is below his expected marginal valuation over the holding period reduces his demand when $\kappa$ increases. ${ }^{15}$ Next, we show how these reallocation effects shape the implications of trading frictions for asset prices and trade volume.

Standard frictionless models emphasize two sets of factors that affect the determination of equilibrium asset prices, i.e., intrinsic properties of the asset and the characteristics of investors

\footnotetext{
${ }^{14}$ These conditions imply that the investor's problem has a solution for all $q>0$, and that the nonnegativity constraints in (6) are slack at every date for every investor in the unique equilibrium. This will simplify the notation but is otherwise inessential for our results. See Lagos and Rocheteau (2006) for utility specifications that do not satisfy the Inada conditions.

${ }^{15}$ In Appendix B, we show that this insight does not rely on preference shocks being independently and identically distributed (i.i.d.). We derive an expression analogous to (23) when preference shocks follow a general Markov process and provide several sufficient conditions that allow us to sign $\partial g_{i}(\kappa, p) / \partial \kappa$. We show, for instance, that for $\kappa$ sufficiently large, $\partial g_{i}(\kappa, p) / \partial \kappa>0$ if and only if $u_{i}^{\prime}\left(a_{i}\right)<\sum_{j=1}^{I} \pi_{i j} u_{j}^{\prime}\left(a_{i}\right)$, where $\pi_{i j}$ is the probability that an investor with preference type $i$ draws type $j$ conditional on his receiving a preference shock. This condition is equivalent to the condition in part $(i)$ of Proposition 2 in Gârleanu (2006). See Proposition 9 in Appendix B for details.
} 
who buy it. Search theory identifies a third element: the manner in which the asset is traded, i.e., the details of the microstructure of the asset market, such as the rate at which investors contact dealers and the market power of dealers. The following proposition characterizes the effects of these trading frictions on asset prices.

Proposition 4 Let $u_{i}(a)=\varepsilon_{i} u(a)$. If $\left[u^{\prime}(a)\right]^{2} / u^{\prime \prime}(a)$ is strictly decreasing in a, then $d p / d \kappa>0$. If $\left[u^{\prime}(a)\right]^{2} / u^{\prime \prime}(a)$ is increasing in a, then $d p / d \kappa \leq 0$ (with "=" if $\left[u^{\prime}(a)\right]^{2} / u^{\prime \prime}(a)$ is constant).

For the class of preferences in Proposition $4, \bar{u}_{i}(a)=\bar{\varepsilon}_{i} u(a)$, where $\bar{\varepsilon}_{i}=\frac{(r+\kappa) \varepsilon_{i}+\delta \bar{\varepsilon}}{r+\kappa+\delta}$ and $\bar{\varepsilon}=$ $\sum_{j=1}^{I} \pi_{j} \varepsilon_{j}$, and $(22)$ becomes $\bar{\varepsilon}_{i} u^{\prime}\left(a_{i}\right)=r p$. For a given $p$, the demands of investors with relatively low valuations $\left(\varepsilon_{i}<\bar{\varepsilon}\right)$ fall, while those of investors with high valuations $\left(\varepsilon_{i}>\bar{\varepsilon}\right)$ rise as $\kappa$ increases. Whether an increase in $\kappa$ causes the asset price to rise depends on the curvature of the individual demand for the asset as a function of $\bar{\varepsilon}_{i}$, i.e., on the slope of $\partial a_{i} / \partial \bar{\varepsilon}_{i}=-\left[u^{\prime}\left(a_{i}\right)\right]^{2} /\left[u^{\prime \prime}\left(a_{i}\right) r p\right]$. If $u$ is not too concave, $a_{i}$ is a convex function of $\bar{\varepsilon}_{i}$. For this case, Jensen's inequality implies that the increases in $a_{i}$ for relatively large values of $\varepsilon_{i}$ outweigh the decreases in $a_{i}$ for relatively low values of $\varepsilon_{i}$, and the aggregate demand for the asset increases in response to an increase in $\kappa$. In turn, this implies that the equilibrium price of the asset increases with $\kappa$. Conversely, the asset price is decreasing in $\kappa$ if $u$ is sufficiently concave. For example, if $u(a)=a^{1-\sigma} /(1-\sigma)$ with $\sigma>0$, then $d p / d \kappa<0(>0)$ if $\sigma>1$ $(<1)$. $^{16}$

It is clear from (23) that regardless of the ultimate effect of trading frictions on the asset price, an increase in $\kappa$ induces high-valuation investors to take larger positions and low-valuation investors to take smaller positions. This seems to suggest that the distribution of asset holdings will spread out if frictions are reduced. However, this intuition is only partial because (23) keeps the equilibrium asset price constant. In the following proposition we characterize the general equilibrium effect of trading frictions on the dispersion of the distribution of asset holdings.

Proposition 5 (i) Let $u_{i}(a)=\varepsilon_{i} a^{1-\sigma} /(1-\sigma)$ with $\sigma>0$. An increase in $\kappa$ causes the equilibrium distribution of asset holdings to become riskier, in the second-order stochastic sense.

(ii) For all $i \in\{1, . ., I\}, a_{i} \rightarrow A$ as $r+\kappa \rightarrow 0$.

\footnotetext{
${ }^{16}$ If $u(a)=\log a$, then $a_{i}$ is linear in $\bar{\varepsilon}_{i}$ and $d p / d \kappa=0$. This particular result is reminiscent of the findings in Constantinides (1986), Gârleanu (2006), and Heaton and Lucas (1995) that the equilibrium asset price is not (much) affected by transaction costs. In Appendix B, we show that this finding generalizes to the more general case of Markovian preference shocks.
} 
Part $(i)$ of Proposition 5 confirms that for a particular class of preferences, the distribution of asset holdings spreads out if frictions are reduced. According to part $(i i)$, the dispersion of the distribution of asset holdings approaches zero as trading frictions become very severe, provided that investors are sufficiently patient. This result holds for general preferences and will be useful in our analysis of transaction costs and trade volume. ${ }^{17}$

Let $\mathcal{V}$ denote trade volume, defined as

$$
\mathcal{V}=\frac{\alpha}{2} \sum_{i, j=1}^{I} n_{i j}\left|a_{j}-a_{i}\right| .
$$

An increase in $\kappa$ has three distinct effects on $\mathcal{V}$. First, the measure of investors in any individual state $(i, j) \in \mathbb{X}^{2}$ who gain access to the market and are able to trade increases, which tends to increase $\mathcal{V}$. Second, the proportion $1-\sum_{i=1}^{I} n_{i i}$ of agents who are mismatched to their asset position - the fraction of agents who wish to trade - decreases, which tends to decrease $\mathcal{V}$. Finally, the distribution of asset holdings spreads out, which tends to increase the quantity of assets traded in many individual trades. With (21) and (24), it is possible to show that the first two effects combined lead to an increase in $\mathcal{V}$. Although it is difficult to sign the third effect in general due to the general equilibrium effects of the price on the distribution of asset holdings, we provide analytical results for three special cases. First, with $I=2$ it is possible to show that an increase in $\kappa$ unambiguously leads to an increase in $\mathcal{V}$. The second special case allows for richer heterogeneity in types, but adopts a specification of preferences for which the equilibrium asset price is independent of trading frictions. The third case considers the limiting economy where trading frictions become very severe and investors are patient.

Proposition 6 (i) Let $u_{i}(a)=\varepsilon_{i} a^{1-\sigma} /(1-\sigma)$ with $\sigma>0$, and assume that $I=2$. Trade volume increases with $\kappa$.

(ii) Let $u_{i}(a)=\varepsilon_{i} \ln a$. Trade volume increases with $\kappa$. Moreover, for any pair $\left(\kappa, \kappa^{\prime}\right)$ such that $\kappa^{\prime}>\kappa$, the distribution of trade sizes associated with $\kappa^{\prime}$ first-order stochastically dominates the one associated with $\kappa$.

\footnotetext{
${ }^{17}$ In part (iii) of Proposition 11 (Appendix B), we show that part (ii) of Proposition 5 also holds for more general preference shock processes. The proof of part $(i)$ of Proposition 5 relies on the assumption of i.i.d. preference shocks and its immediate mean-reverting property. The i.i.d. specification, however, is without loss of generality for the case $I=2$. (This is the case analyzed by DGP and much of the subsequent literature.) For $I>2$, an increase in trading frictions need not compress the cross-sectional distribution of asset holdings. As pointed out by Gârleanu (2006), it is possible that for certain ranges of $\kappa$, an investor with a high current valuation (relative to the cross-section of current valuations) may increase his asset holdings in response to an increase in trading frictions. The general insight, however, is that investors always react to more severe trading frictions by choosing asset positions that reduce the expected sizes of their future asset reallocations.
} 
(iii) Trade volume approaches zero as $r+\kappa \rightarrow 0$.

\section{Transaction costs}

Intermediation fees and the implied bid-ask spreads constitute the out-of-pocket transaction costs borne by investors and are commonly used measures of market liquidity. ${ }^{18}$ At the same time, these spreads determine the revenue of dealers, and hence are a key determinant of their incentives to make markets and provide liquidity — a theme we explore in Section 6.

Intermediation fees depend on the rate at which investors can contact alternative dealers, on their bargaining power in bilateral negotiations, and on the size of the trade. The following result shows that, keeping the characteristics of an investor and a dealer constant, transaction costs - both total and per unit of asset traded - increase with the size of the trade. ${ }^{19}$

Lemma 4 Consider an investor who holds asset position $a \geq 0$ and wishes to trade $\left|a_{i}-a\right|>0$. Both $\frac{\partial \phi_{i}(a)}{\partial a}$ and $\frac{\partial}{\partial a}\left[\frac{\phi_{i}(a)}{\left|a_{i}-a\right|}\right]$ have the same sign as $a-a_{i}$.

In the general equilibrium, trading frequencies and bargaining power affect transaction costs through three channels. Consider for example $\phi_{i}\left(a_{j}\right)$, the fee paid by an investor who currently has preference type $i$, and whose preference type was $j$ at the time of his last trade. A larger $\alpha$ tends to reduce the fees that dealers can extract for any given trade size (e.g., it increases the denominator of (20)). Intuitively, a larger $\alpha$ implies better search options for the investorthe competition effect of reduced trading frictions emphasized in the previous literature. But here an increase in $\alpha$ also changes the investor's expected utility from holding his current asset position, $a_{j}$, relative to the expected utility from holding his desired asset position, $a_{i}$ (i.e., it changes $\bar{u}_{i}$ in (20)). This effect may decrease or increase the intermediation fee depending on the specific values of $a_{j}$ and $a_{i}$. Finally, $\alpha$ affects the actual and desired asset positions, $a_{j}$ and $a_{i}$, themselves. A larger $\alpha$ can induce investors to conduct larger asset reallocations (Proposition

\footnotetext{
${ }^{18}$ See footnote 9 for the theoretical link between intermediation fees and bid-ask spreads.

${ }^{19}$ The theory generates a distribution of transaction costs, not only across trade-size categories, but also among trades of equal size, which is in accordance with the evidence from the OTC market for municipal bonds (Green, Hollifield, and Schurhoff, 2007). The increasing relationship between trade size and transaction cost for given $\alpha$ is consistent with the empirical evidence on foreign exchange markets (Burnside, Eichenbaum, Kleshchelski, and Rebelo, 2006, Table 12). In contrast, empirical studies on municipal and corporate bond markets document that larger trades tend to be executed at a discount (Harris and Piwowar, 2006). Our model can rationalize this observation if we allow for heterogeneous investors, some of which can contact dealers faster than others. See Lagos and Rocheteau (2006).
} 
6) and by Lemma 4 this translates into larger fees for dealers, on average - the reallocation effect of reduced trading frictions. These three effects can give rise to nonmonotonicities in the dealers' incentives to make markets in response to changes in the degree of trading frictions. We prove this result for the case of patient traders, both for intermediation fees for individual trades (Proposition 7) and for market-wide measures of transaction costs (Corollary 1).

Proposition 7 For each $(i, j) \in \mathbb{X}^{2}$, there exists $\bar{r}>0$, such that for all $r<\bar{r}$ and $\eta \in(0,1)$, $\phi_{j i}$ is nonmonotonic in $\kappa$ and is largest for some $\kappa \in(0, \infty)$.

In very illiquid markets (as $r+\kappa \rightarrow 0$ ), investors hedge against future preference shocks by choosing asset holdings that reflect their average utility from holding the asset rather than their current utility at the time they trade. Thus, trade sizes and fees are small. In very liquid markets (as $\kappa \rightarrow \infty$ ) investors trade large quantities, but the fees they pay are also small because of favorable search options. For intermediate values of $\kappa$, trade sizes are considerable and dealers have a degree of market power that results in larger intermediation fees.

The average fee charged by dealers across the various types of trades is $\Phi=\sum_{i, j=1}^{I} n_{j i} \phi_{j i}$, or using (20),

$$
\Phi=\eta \sum_{i, j=1}^{I} n_{j i} \frac{\bar{u}_{i}\left(a_{i}\right)-\bar{u}_{i}\left(a_{j}\right)}{r+\kappa} .
$$

This average fee is the expected revenue of an individual dealer conditional on meeting an investor, and is therefore a key determinant of the dealers' incentives to make markets. The following corollary of Proposition 7 characterizes how trading frictions affect these incentives, which will play a key role in the following section.

Corollary 1 There exists $\hat{r}>0$, such that for all $r<\hat{r}$ and $\eta \in(0,1)$, $\Phi$ is nonmonotonic in $\kappa$ and is largest for some $\kappa \in(0, \infty)$.

Corollary 1 says that dealers are better off when they trade in markets that are neither too liquid nor too illiquid. If $\kappa$ is very large, dealers would find it profitable to shift the trading activity to markets with larger $\eta$ or smaller $\alpha$. Conversely if $\kappa$ is very small, perhaps surprisingly, dealers would benefit from reductions in $\eta$ or increases in $\alpha$. 


\section{Endogenous execution delays}

In this section we allow for free entry of dealers in order to endogenize the supply of liquidity services and the length of the trading delays. We formalize the notion that a dealer's profit depends on the competition for order flow that he faces from other dealers.

Suppose that $\alpha$ is a continuously differentiable function of the measure of dealers in the market, $v$, with $\partial \alpha(v) / \partial v>0$ and $\partial[\alpha(v) / v] / \partial v<0$. We also specify $\alpha(0)=0, \lim _{v \rightarrow \infty} \alpha(v)=\infty$ and $\lim _{v \rightarrow \infty} \alpha(v) / v=0$. Since all matches are bilateral and random, the Poisson rate at which a dealer contacts an investor is $\alpha(v) / v$. For larger $v$, each investor contacts dealers faster, but the order flow decreases for each individual dealer.

There is a large measure of dealers who can choose to participate in the market. Dealers who choose to operate incur a flow cost $\gamma>0$ that represents the ongoing costs of running the dealership. ${ }^{20}$ With (25), the free-entry condition, $\frac{\alpha(v)}{v} \Phi=\gamma$, can be written as

$$
\frac{\alpha(v)}{v} \eta \sum_{i, j=1}^{I} n_{j i} \frac{\bar{u}_{i}\left(a_{i}\right)-\bar{u}_{i}\left(a_{j}\right)}{r+\alpha(v)(1-\eta)}=\gamma .
$$

A steady-state equilibrium with free entry is a list $\left\langle\left\{a_{i}\right\}, q, p,\left\{\phi_{i}(a)\right\},\left\{n_{j i}\right\}, v\right\rangle$ that satisfies (18)-(21) and (26), with $\alpha=\alpha(v)$. It can be shown (see Lagos and Rocheteau, 2006) that for any $\eta>0$ there exists a steady-state equilibrium with free entry of dealers, and it has $v>0$.

The steady-state equilibrium with free entry need not be unique. Although the measure of dealers, $v$, is strictly increasing in $\Phi$, according to Corollary 1 the dealers' expected revenue, $\Phi$, can itself be a nonmonotonic function of $\alpha(v)$. On the one hand, faster trade means more competition among dealers, which tends to reduce intermediation fees. But an increase in $\alpha(v)$ also induces investors to take on more extreme asset positions, which means that on average, dealers will intermediate larger trades and earn higher fees. For the case of patient traders, the following result shows that the model necessarily exhibits multiple steady-state equilibria if $\alpha(v) / v$ is not too elastic (the effect of an additional dealer on existing dealers' order flow is not too large) and $\gamma$ is in an intermediate range.

Proposition 8 Assume $\eta \in(0,1)$ and $\alpha(v)=v^{\theta}$, with $\theta \in(0,1)$. There exist $\tilde{r}>0, \tilde{\theta} \in(0,1)$, $\bar{\gamma}>0$, and $\underline{\gamma} \in(0, \bar{\gamma})$ such that for all $(r, \theta) \in(0, \tilde{r}) \times(\tilde{\theta}, 1)$, there are multiple steady-state equilibria if $\gamma \in(\underline{\gamma}, \bar{\gamma})$.

\footnotetext{
${ }^{20}$ Our formulation of the free entry of dealers is analogous to the free entry of firms in Pissarides (2000).
} 
In the case of multiple equilibria, the market could be trapped in a low-liquidity equilibrium where few dealers enter and investors engage in relatively small transactions. ${ }^{21}$ Regarding the efficiency properties of equilibrium with entry, investors' asset holdings are efficient only if dealers have no bargaining power, just as in the formulation with a fixed population of dealers. Therefore, since there is no equilibrium with $v>0$ when $\eta=0$, an equilibrium with entry is always inefficient. ${ }^{22}$

\section{Conclusion}

We have developed a model of trade in asset markets that contributes to a growing literature that uses search theory to model the trading frictions characteristic of OTC markets. A novel aspect of our theory is that it does not assume restrictions on asset holdings and therefore allows market participants to accommodate trading frictions by adjusting their asset positions so as to reduce their trading needs. We have found that this mechanism has important implications for market efficiency and the way in which trading frictions shape asset prices as well as standard measures of financial liquidity. Although we have emphasized the application to OTC markets for financial securities, the structure and solution techniques we have developed should prove useful for applications of search theory to other contexts where idiosyncratic uncertainty and random matching give rise to nontrivial distributions of asset holdings.

\footnotetext{
${ }^{21}$ The strategic complementarity that leads to multiple equilibria in this model depends crucially on the endogenous distribution of asset holdings. The multiplicity is not due to increasing returns in the meeting technology, as in Diamond (1982) or Vayanos and Weill (2008), or to the cost of holding the liquid asset, as in Rocheteau and Wright (2005).

${ }^{22}$ As $r \rightarrow 0$, it can be shown that an equilibrium with free entry is efficient if and only if $\eta=0$ and $v \alpha^{\prime}(v) / \alpha(v)=\eta$. Entry introduces a negative externality on other dealers' order flow, and this externality is internalized if and only if the elasticity of the contact technology $\alpha(v)$ coincides with dealers' bargaining power-the so-called Hosios (1990) condition.
} 


\section{A Proofs}

Proof of Lemma 1. We can rewrite (5) as

$$
V_{i}(a, t)=\bar{U}_{i}(a)+\mathbb{E}_{i}\left[e^{-r\left(T_{\kappa}-t\right)}\left\{p\left(T_{\kappa}\right) a+\max _{a^{\prime} \geq 0}\left[V_{k\left(T_{\kappa}\right)}\left(a^{\prime}, T_{\kappa}\right)-p\left(T_{\kappa}\right) a^{\prime}\right]\right\}\right]
$$

where

$$
\bar{U}_{i}(a)=\mathbb{E}_{i}\left[\int_{t}^{T_{\kappa}} e^{-r(s-t)} u_{k(s)}(a) d s\right]=\mathbb{E}_{i}\left[\int_{0}^{T_{\kappa}-t} e^{-r s} u_{k(t+s)}(a) d s\right] .
$$

From (27), the problem of an investor with preference shock $i$ who gains access to the market at time $t$ is given by

$$
\max _{a^{\prime} \geq 0}\left[\bar{U}_{i}\left(a^{\prime}\right)-\left\{p(t)-\mathbb{E}\left[e^{-r\left(T_{\kappa}-t\right)} p\left(T_{\kappa}\right)\right]\right\} a^{\prime}\right] .
$$

We proceed in two steps: $(i)$ calculate $\bar{U}_{i}(a)$, and $(i i)$ calculate $\mathbb{E}\left[e^{-r T_{\kappa}} p\left(t+T_{\kappa}\right)\right]$.

Step $(i)$. Equation $(28)$ can be written recursively as

$$
(r+\kappa) \bar{U}_{i}(a)=u_{i}(a)+\delta \sum_{j=1}^{I} \pi_{j}\left[\bar{U}_{j}(a)-\bar{U}_{i}(a)\right] .
$$

(We provide an alternative, more detailed, derivation in Lagos and Rocheteau (2006).) Multiply

(30) through by $\pi_{i}$, sum over $i$, solve for $\sum_{j} \pi_{j} \bar{U}_{j}(a)$, and substitute this expression back into (30) to obtain $\bar{U}_{i}(a)=\frac{\bar{u}_{i}(a)}{r+\kappa}$, where $\bar{u}_{i}(a)$ is as in (7).

Step $(i i)$. The expected discounted price of the asset at the next time when the investor gets an opportunity to trade is

$$
\mathbb{E}\left[e^{-r\left(T_{\kappa}-t\right)} p\left(T_{\kappa}\right)\right]=\kappa \int_{0}^{\infty} e^{-(r+\kappa) s} p(t+s) d s .
$$

Finally, substitute $\bar{U}_{i}(a)=\frac{\bar{u}_{i}(a)}{r+\kappa}$ and (31) into (29) and multiply through by $(r+\kappa)$ to obtain the formulation of the investor's problem in the statement of the lemma.

Proof of Lemma 2. Rewrite (8) as

$$
q(t)=(r+\kappa)\left[p(t)-\kappa \int_{t}^{\infty} e^{-(r+\kappa)(s-t)} p(s) d s\right]
$$

and differentiate with respect to $t$ to obtain

$$
r p(t)-\dot{p}(t)=q(t)-\frac{\dot{q}(t)}{r+\kappa} .
$$

Integrate this expression forward and use $\lim _{t \rightarrow \infty} e^{-r t} p(t)=0$ to arrive at (10). 
Proof of Lemma 3. We proceed in three steps: $(i)$ derive $n_{j i}(\tau, t),(i i)$ derive $n_{j i}^{0}(\mathcal{A}, t)$, and (iii) obtain $H_{t}(\mathcal{A}, \mathcal{I})$ for an arbitrary $(\mathcal{A}, \mathcal{I}) \in \Sigma$.

Step $(i)$. The density measure of investors who last readjusted their asset holdings at time $t-\tau>0$ is $\alpha e^{-\alpha \tau}$. The probability that an investor who last contacted a dealer at time $t-\tau$ has a history of preference types involving $k(t-\tau)=j$ and $k(t)=i$ is $\left(1-e^{-\delta \tau}\right) \pi_{i}+\mathbb{I}_{\{i=j\}} e^{-\delta \tau}$. Since the measure of investors with preference type $j$ at time $t-\tau$ is $n_{j}(t-\tau)$, and the Poisson process for meeting dealers and the compound Poisson process for preference shocks are independent, the density measure of investors who last traded at time $t-\tau$ and who have a history of preferences involving $k(t-\tau)=j$ and $k(t)=i$, is $n_{j i}(\tau, t)=\alpha e^{-\alpha \tau}\left[\left(1-e^{-\delta \tau}\right) \pi_{i}+\mathbb{I}_{\{i=j\}} e^{-\delta \tau}\right] n_{j}(t-\tau)$, as given by (16).

Step (ii). The measure of investors who have not contacted a dealer up to time $t$ is $e^{-\alpha t}$. Since the Poisson meeting process is independent of investors' individual states, the time- $t$ measure of investors whose asset holdings and preference types lay in the set $(\mathcal{A},\{j\})$ at time 0 and who have not yet met a dealer at time $t$ is $e^{-\alpha t} H_{0}(\mathcal{A},\{j\})$. The measure of investors who were of preference type $j$ at time 0 and are of type $i$ at time $t$ is $\left(1-e^{-\delta t}\right) \pi_{i}+e^{-\delta t} \mathbb{I}_{\{j=i\}}$. Thus, the time- $t$ measure of investors who at time 0 had preference type $j$ and assets in $\mathcal{A}$, whose preference type is $i$ at the current time $t$, and who have never traded (so their asset holdings are still in $\mathcal{A})$ is $n_{j i}^{0}(\mathcal{A}, t)=e^{-\alpha t}\left[\left(1-e^{-\delta t}\right) \pi_{i}+e^{-\delta t} \mathbb{I}_{\{j=i\}}\right] H_{0}(\mathcal{A},\{j\})$, as given in $(15)$.

Step $(i i i) . H_{t}(\mathcal{A}, \mathcal{I})$ is the measure of investors who have an individual state $(a, i) \in(\mathcal{A}, \mathcal{I})$ at time $t$. The first term in $H_{t}(\mathcal{A}, \mathcal{I})$ is $\sum_{i \in \mathcal{I}} \sum_{j=1}^{I} n_{j i}^{0}(\mathcal{A}, t)$, namely, those investors who never contacted dealers but who were holding asset positions in the set $\mathcal{A}$ at time 0 and whose preference types at $t$ lie in $\mathcal{I}$. The time- $t$ measure of investors of type $i$ who chose an asset position in the set $\mathcal{A}$ the last time they traded, given that their preference type at that time was $j$, is $\int_{0}^{t} \mathbb{I}_{\left\{a_{j}(t-\tau) \in \mathcal{A}\right\}} n_{j i}(\tau, t) d \tau$. Thus, the second term in $H_{t}(\mathcal{A}, \mathcal{I})$, namely, the measure of investors who the last time they traded chose asset positions that belong to the set $\mathcal{A}$ and whose preference types at time $t$ lie in $\mathcal{I}$, is $\sum_{i \in \mathcal{I}} \sum_{j=1}^{I} \int_{0}^{t} \mathbb{I}_{\left\{a_{j}(t-\tau) \in \mathcal{A}\right\}} n_{j i}(\tau, t) d \tau$.

Proof of Proposition 1. For all $t \geq 0$, the distribution $\left\{n_{i}(t)\right\}_{i=1}^{I}$ is unique and given by (12). Define $A_{t}^{d}(q) \equiv\left\{\sum_{i=1}^{I} n_{i}(t) a_{i}(q): a_{i}(q) \in \arg \max _{a^{\prime} \geq 0}\left[\bar{u}_{i}\left(a^{\prime}\right)-q a^{\prime}\right]\right\}$ for $q \in(\underline{q}(t),+\infty)$, where $\underline{q}(t)=\max _{i \in \mathbb{X}} \bar{u}_{i}^{\prime}(\infty) \mathbb{I}_{\left\{n_{i}(t)>0\right\}}$. (If $q \leq \underline{q}(t)$ then (9) has no solution for some $i$ such that $n_{i}(t)>0$.) Given that $u_{i}$ (and hence $\bar{u}_{i}$ ) is strictly concave and continuously differentiable, $a_{i}(q)$ is uniquely determined for all $q \in(\underline{q}(t),+\infty)$ and all $i$ such that $n_{i}(t)>0$, and it is continuous. Consequently, $A_{t}^{d}(q)$ is singled-valued and continuous for $q \in(\underline{q}(t),+\infty)$. Moreover, (9) implies 
that any interior choice $a_{i}(t)$ is a strictly decreasing function of $q(t)$ for every $i$. Thus, $A_{t}^{d}(q)$ is strictly decreasing for all $q \in(\underline{q}(t), \bar{q}(t))$, where $\bar{q}(t)=\max _{i \in \mathbb{X}} \bar{u}_{i}^{\prime}(0) \mathbb{I}_{\left\{n_{i}(t)>0\right\}}$, and $A_{t}^{d}(q)=\{0\}$ for all $q \geq \bar{q}(t)$. As $q \downarrow \underline{q}(t), A_{t}^{d}(q) \rightarrow+\infty$, and as $q \uparrow \bar{q}(t), A_{t}^{d}(q) \rightarrow 0$. So for each $t$ there is a unique $q(t) \in(\underline{q}(t), \bar{q}(t))$ such that $A_{t}^{d}[q(t)]=\{A\}$ or equivalently, such that $\sum_{i=1}^{I} n_{i}(t) a_{i}[q(t)]=A$. Given this $q(t)$, there is a unique $\left\{a_{i}(t)\right\}_{i=1}^{I}$ that solves $(9)$. Given $q(t),(11)$ gives the fee $\phi_{i}(a, t)$ for every $i$ and $a$. Finally, given $\left\{a_{i}(t)\right\}_{i=1}^{I}$ the distribution $H_{t}$ is given by (14).

Proof of Proposition 2. Calculations similar to those contained in part $(i)$ of the proof of Lemma 1 imply $(r+\alpha) \hat{U}_{i}(a)=\frac{r+\alpha}{r+\alpha+\delta} u_{i}(a)+\frac{\delta}{r+\alpha+\delta} \sum_{j=1}^{I} \pi_{j} u_{j}(a)$. Substitute this expression into the planner's objective functional to get

$$
\max _{\left\{a_{i}(t)\right\}} \int_{0}^{\infty} \frac{\alpha}{r+\alpha}\left\{\sum_{i=1}^{I}\left[\frac{r+\alpha}{r+\alpha+\delta} u_{i}\left[a_{i}(t)\right]+\frac{\delta}{r+\alpha+\delta} \sum_{j=1}^{I} \pi_{j} u_{j}\left[a_{i}(t)\right]\right] n_{i}(t)\right\} e^{-r t} d t
$$

subject to $\sum_{j=1}^{I} n_{j}(t) a_{j}(t) \leq A$ and $a_{i}(t) \geq 0$ for all $i$. Let

$$
\mathcal{L}(t)=\sum_{i=1}^{I}\left[\frac{r+\alpha}{r+\alpha+\delta} u_{i}\left[a_{i}(t)\right]+\frac{\delta}{r+\alpha+\delta} \sum_{k=1}^{I} \pi_{k} u_{k}\left[a_{i}(t)\right]\right] n_{i}(t)+\lambda(t)\left[A-\sum_{i=1}^{I} n_{i}(t) a_{i}(t)\right]
$$

where $\lambda(t)$ is the Lagrange multiplier associated with the resource constraint. Since $\mathcal{L}(t)$ is strictly jointly concave in $\left\{a_{i}(t)\right\}_{i=1}^{I}$, the first-order necessary and sufficient conditions for the problem $\max _{\left\{a_{i}(t)\right\}} \mathcal{L}(t)$ are

$$
\frac{(r+\alpha) u_{i}^{\prime}\left[a_{i}(t)\right]+\delta \sum_{k=1}^{I} \pi_{k} u_{k}^{\prime}\left[a_{i}(t)\right]}{r+\alpha+\delta} \leq \lambda(t), \quad \text { " }=" \quad \text { if } a_{i}(t)>0,
$$

for $i=1, \ldots, I$. The resource constraint (17) at equality is

$$
\sum_{i=1}^{I} n_{i}(t) a_{i}^{*}[\lambda(t)]=A
$$

where $a_{i}^{*}[\lambda(t)]$ is the $a_{i}$ that satisfies (32). Comparing (33) with (13), (32) with (9), and setting $q(t)=\lambda(t)$, it becomes clear that (9) coincides with (32) if and only if $\eta=0$.

Proof of Proposition 3. From (12), $\lim _{t \rightarrow \infty} n_{i}(t)=\pi_{i}$ for each $i$. By an argument similar to the one in the proof of Proposition 1, one can establish that there is a unique, time-invariant, $q$ that clears the asset market. Given this $q$, (9) implies a unique set of time-invariant optimal asset 
holdings $\left\{a_{i}\right\}_{i=1}^{I}$. Thus, $\left\{a_{i}\right\}_{i=1}^{I}$ and $q$ satisfy (18) and (19). Given the fact that $q(t)=q$ for all $t$, (10) implies $p=q / r$. Given $q$ and $\left\{a_{i}\right\}_{i=1}^{I}$, (11) implies (20), which determines the time invariant fees $\left\{\phi_{i}(a)\right\}_{i=1}^{I}$. To derive (21), start from Lemma 3 and note that $\lim _{t \rightarrow \infty} n_{j i}^{0}(\mathcal{A}, t)=0$ for all $i, j \in \mathbb{X}$ and all $\mathcal{A} \subseteq \mathbb{R}_{+}$. Also, $\lim _{t \rightarrow \infty} n_{j i}(\tau, t)=\alpha e^{-\alpha \tau}\left[\left(1-e^{-\delta \tau}\right) \pi_{i}+e^{-\delta \tau} \mathbb{I}_{\{i=j\}}\right] \pi_{j} \equiv$ $n_{j i}(\tau, \infty)$ and $\lim _{t \rightarrow \infty} a_{j}(t-\tau)=a_{j}$, so

$$
\lim _{t \rightarrow \infty} H_{t}(\mathcal{A}, \mathcal{I})=\sum_{i \in \mathcal{I}} \sum_{j=1}^{I}\left[\int_{0}^{\infty} \mathbb{I}_{\left\{a_{j} \in \mathcal{A}\right\}} n_{j i}(\tau, \infty) d \tau\right] \equiv H(\mathcal{A}, \mathcal{I})
$$

for all $(\mathcal{A}, \mathcal{I}) \in \Sigma$. To conclude, observe that $H\left(\left\{a_{i}\right\},\{j\}\right)=\int_{0}^{\infty} n_{i j}(\tau, \infty) d \tau$ and carry out the integration to obtain (21).

Proof of Proposition 4. Differentiate (19) to obtain

$$
\frac{d p}{d \kappa}=\frac{\sum_{i=1}^{I} \pi_{i} \partial a_{i} / \partial \kappa}{-\sum_{i=1}^{I} \pi_{i} \partial a_{i} / \partial p}
$$

The denominator of this expression is strictly positive (from (22)), so focus on the sign of the numerator. Differentiate $(22)$ to obtain $\partial a_{i} / \partial \kappa$, multiply by $\pi_{i}$, and add over all $i$ to arrive at

$$
\sum_{i=1}^{I} \pi_{i} \frac{\partial a_{i}}{\partial \kappa}=\frac{\delta}{(r+\kappa+\delta)^{2} r p} \sum_{i=1}^{I} \pi_{i} \frac{\left[u^{\prime}\left(a_{i}\right)\right]^{2}}{-u^{\prime \prime}\left(a_{i}\right)}\left(\varepsilon_{i}-\bar{\varepsilon}\right) .
$$

Suppose $-\left[u^{\prime}(a)\right]^{2} / u^{\prime \prime}(a)$ is strictly increasing in $a$. Let $\bar{a}$ denote the $a$ that solves $(22)$ for $\bar{\varepsilon}_{i}=\bar{\varepsilon}$. Then, note that $-\left[u^{\prime}\left(a_{i}\right)\right]^{2}\left(\varepsilon_{i}-\bar{\varepsilon}\right) / u^{\prime \prime}\left(a_{i}\right) \geq-\left[u^{\prime}(\bar{a})\right]^{2}\left(\varepsilon_{i}-\bar{\varepsilon}\right) / u^{\prime \prime}(\bar{a})$ for each $i$, with strict inequality for all $i$ such that $\varepsilon_{i} \neq \bar{\varepsilon}$. Thus, $\sum_{i=1}^{I} \pi_{i} \frac{\partial a_{i}}{\partial \kappa}>0$ and consequently, $\frac{d p}{d \kappa}>0$. Similar reasoning implies $\frac{d p}{d \kappa}<0$ if $-\left[u^{\prime}(a)\right]^{2} / u^{\prime \prime}(a)$ is strictly decreasing and $\frac{d p}{d \kappa}=0$ if $-\left[u^{\prime}(a)\right]^{2} / u^{\prime \prime}(a)$ is constant in $a$.

Proof of Proposition 5. (i) Let $a_{i}(\kappa)$ denote the individual demand of an investor with preference type $i$ in a market with effective contact rate $\kappa$. With $u_{i}(a)=\varepsilon_{i} a^{1-\sigma} /(1-\sigma)$,

$$
a_{i}(\kappa)=\frac{A}{\sum_{j=1}^{I} \pi_{j}\left[\frac{(r+\kappa) \varepsilon_{j}+\delta \bar{\varepsilon}}{(r+\kappa) \varepsilon_{i}+\delta \bar{\varepsilon}}\right]^{1 / \sigma}} .
$$

Consider $\kappa^{\prime}>\kappa$. We have $a_{1}\left(\kappa^{\prime}\right)<a_{1}(\kappa)$, since $\frac{\left(r+\kappa^{\prime}\right) \varepsilon_{j}+\delta \bar{\varepsilon}}{\left(r+\kappa^{\prime}\right) \varepsilon_{1}+\delta \bar{\varepsilon}}>\frac{(r+\kappa) \varepsilon_{j}+\delta \bar{\varepsilon}}{(r+\kappa) \varepsilon_{1}+\delta \bar{\varepsilon}}$ for all $j>1$, and $a_{I}\left(\kappa^{\prime}\right)>a_{I}(\kappa)$, since $\frac{\left(r+\kappa^{\prime}\right) \varepsilon_{j}+\delta \bar{\varepsilon}}{\left(r+\kappa^{\prime}\right) \varepsilon_{I}+\delta \bar{\varepsilon}}<\frac{(r+\kappa) \varepsilon_{j}+\delta \bar{\varepsilon}}{(r+\kappa) \varepsilon_{I}+\delta \bar{\varepsilon}}$ for all $j<I$. The difference $a_{i}\left(\kappa^{\prime}\right)-a_{i}(\kappa)$ is continuous in $\varepsilon_{i}$, so there exists $\tilde{\varepsilon} \in\left(\varepsilon_{1}, \varepsilon_{I}\right)$ such that $a_{i}\left(\kappa^{\prime}\right)=a_{i}(\kappa) \equiv \tilde{a}$. Moreover, from (34),

$$
\left.\frac{\partial a_{i}\left(\kappa^{\prime}\right)}{\partial \varepsilon_{i}}\right|_{\varepsilon_{i}=\tilde{\varepsilon}}=\frac{\left(r+\kappa^{\prime}\right) \tilde{a}}{\sigma\left[\left(r+\kappa^{\prime}\right) \tilde{\varepsilon}+\delta \bar{\varepsilon}\right]}>\frac{(r+\kappa) \tilde{a}}{\sigma[(r+\kappa) \tilde{\varepsilon}+\delta \bar{\varepsilon}]}=\left.\frac{\partial a_{i}(\kappa)}{\partial \varepsilon_{i}}\right|_{\varepsilon_{i}=\tilde{\varepsilon}}
$$


so $a_{i}\left(\kappa^{\prime}\right)$ as a function of $\varepsilon_{i}$ intersects $a_{i}(\kappa)$ from below. Hence $\tilde{\varepsilon}$ is unique, and $a_{i}\left(\kappa^{\prime}\right)<a_{i}(\kappa)$ for all $\varepsilon_{i}<\tilde{\varepsilon}$ and $a_{i}\left(\kappa^{\prime}\right)>a_{i}(\kappa)$ for all $\varepsilon_{i}>\tilde{\varepsilon}$. With (21), the cumulative distribution of assets indexed by $\kappa$, is $\mathbb{G}_{\kappa}(a)=\sum_{j=1}^{I} \mathbb{I}_{\left\{a_{j}(\kappa) \leq a\right\}} \pi_{j}$. The fact that $a_{i}\left(\kappa^{\prime}\right)<a_{i}(\kappa)$ for $\varepsilon_{i}<\tilde{\varepsilon}$ implies $\mathbb{G}_{\kappa^{\prime}}(a) \geq \mathbb{G}_{\kappa}(a)$ for all $a<\tilde{a}$. Thus, $\int_{0}^{a} \mathbb{G}_{\kappa^{\prime}}(x) d x \geq \int_{0}^{a} \mathbb{G}_{\kappa}(x) d x$ for all $a<\tilde{a}$. Moreover, $\int_{0}^{a_{I}\left(\kappa^{\prime}\right)} \mathbb{G}_{\kappa^{\prime}}(x) d x=\int_{0}^{a} a_{I}\left(\kappa^{\prime}\right) \mathbb{G}_{\kappa}(x) d x$, so $\int_{0}^{a} \mathbb{G}_{\kappa^{\prime}}(x) d x-\int_{0}^{a} \mathbb{G}_{\kappa}(x) d x=\int_{a}^{\infty} \mathbb{G}_{\kappa}(x) d x-\int_{a}^{\infty} \mathbb{G}_{\kappa^{\prime}}(x) d x$. The right side of this expression is nonnegative for $a \geq \tilde{a}$ because $a_{i}\left(\kappa^{\prime}\right) \geq a_{i}(\kappa)$ for $\varepsilon_{i} \geq \tilde{\varepsilon}$. Thus, $\int_{0}^{a} \mathbb{G}_{\kappa^{\prime}}(x) d x \geq \int_{0}^{a} \mathbb{G}_{\kappa}(x) d x$ for all $a \geq \tilde{a}$. We conclude $\int_{0}^{a} \mathbb{G}_{\kappa^{\prime}}(x) d x \geq \int_{0}^{a} \mathbb{G}_{\kappa}(x) d x$ for all $a \geq 0$, i.e., $\mathbb{G}_{\kappa}$ second-order stochastically dominates $\mathbb{G}_{\kappa^{\prime}}$.

(ii) From (7), as $r+\kappa \rightarrow 0$ then $\bar{u}_{i}(a) \rightarrow \delta \sum_{j=1}^{I} \pi_{j} u_{j}(a)$, which is independent of $i$. Together with market clearing, this implies that $a_{i} \rightarrow A$ for all $i \in\{1, . ., I\}$ as $r+\kappa \rightarrow 0$.

Proof of Proposition 6. (i) For $I=2$, we have $\mathbb{X}=\{1,2\}$ and

$$
\mathcal{V}=\frac{\alpha \delta \pi_{1} \pi_{2}}{\alpha+\delta}\left[a_{2}(\kappa)-a_{1}(\kappa)\right]
$$

where $a_{i}(\kappa)$ is given by $(34)$. Since $\varepsilon_{1}<\varepsilon_{2}$, we have $a_{1}(\kappa)<a_{2}(\kappa)$, and by part $(i)$ of Proposition $5, \frac{d a_{1}(\kappa)}{d \kappa}<0<\frac{d a_{2}(\kappa)}{d \kappa}$. To find $\frac{d \mathcal{V}}{d \kappa}$, we consider two cases. (a) An increase in $\kappa$ caused by a decrease in $\eta$ (keeping $\alpha$ constant). For this case, $\frac{d \mathcal{V}}{d \kappa}=\frac{\alpha \delta \pi_{1} \pi_{2}}{\alpha+\delta}\left[\frac{d a_{2}(\kappa)}{d \kappa}-\frac{d a_{1}(\kappa)}{d \kappa}\right]>0$. (b) An increase in $\kappa$ caused by an increase in $\alpha$, which implies $\frac{d \mathcal{V}}{d \kappa}=\left(\frac{\delta}{\alpha+\delta}\right)^{2} \pi_{1} \pi_{2}\left[a_{2}(\kappa)-a_{1}(\kappa)\right]+$ $\frac{\alpha \delta \pi_{1} \pi_{2}}{\alpha+\delta}\left[\frac{d a_{2}(\kappa)}{d \kappa}-\frac{d a_{1}(\kappa)}{d \kappa}\right]>0$.

(ii) Since $u_{i}(a)=\varepsilon_{i} \ln a$, we have $a_{i}>0$ for all $i$, and $a_{i} \neq a_{j}$ unless $i=j$. From (21), the proportion of trades that involve buying $a_{i}$ and selling $a_{j}$ or vice versa (for $i \neq j$ ) is $\left(n_{i j}+n_{j i}\right) /\left(1-\sum_{i=1}^{I} n_{i i}\right)=2 \pi_{i} \pi_{j} /\left(1-\sum_{i=1}^{I} \pi_{i}^{2}\right)$, which is independent of $\kappa$. From Proposition $4, d p / d \kappa=0$, so differentiating $(22)$,

$$
\frac{d\left[g_{i}(\kappa ; p)-g_{j}(\kappa ; p)\right]}{d \kappa}=\frac{\delta\left(\varepsilon_{i}-\varepsilon_{j}\right)}{r p(r+\kappa+\delta)^{2}} .
$$

Thus, $\left|a_{i}-a_{j}\right|=\left|g_{i}(\kappa ; p)-g_{j}(\kappa ; p)\right|$ increases with $\kappa$ for all $i \neq j$. The measure of trades of size less than $z \geq 0$ is

$$
\sum_{i=1}^{I} \sum_{j \neq i} \frac{\pi_{i} \pi_{j}}{1-\sum_{i=1}^{I} \pi_{i}^{2}} \mathbb{I}_{\left\{\left|a_{i}-a_{j}\right| \leq z\right\}},
$$

which is decreasing in $\kappa$. This establishes that the distribution of trade sizes associated with $\kappa^{\prime}$ first-order stochastically dominates the one associated with $\kappa$ if $\kappa^{\prime}>\kappa$. Since every trade size is larger in the market with a larger $\kappa$, we conclude that $\mathcal{V}$ increases with $\kappa$. 
(iii) This follows immediately from (24) and part (ii) of Proposition 5.

Proof of Lemma 4. Differentiate (20) to obtain

$$
\frac{\partial \phi_{i}(a)}{\partial a}=-\frac{\eta}{r+\kappa}\left[\bar{u}_{i}^{\prime}(a)-q\right]
$$

Suppose that the nonnegativity constraint on $a_{i}$ is slack. Then, since $\bar{u}_{i}$ is strictly concave and $\bar{u}_{i}^{\prime}\left(a_{i}\right)-q=0$, we know that $\bar{u}_{i}^{\prime}(a)-q<0$ if and only if $a-a_{i}>0$, and $\frac{\partial \phi_{i}(a)}{\partial a}$ has the same sign as $a-a_{i}$. If $a_{i}=0$, then $a>a_{i}$ and $\bar{u}_{i}^{\prime}(a)-q<\bar{u}_{i}^{\prime}\left(a_{i}\right)-q \leq 0$, so $\frac{\partial \phi_{i}(a)}{\partial a}>0$, which is the same sign as $a-a_{i}=a>0$. This establishes the first part. To show the second part, divide (20) by $\left(a_{i}-a\right)$ and differentiate the resulting expression to get

$$
\frac{\partial}{\partial a}\left[\frac{\phi_{i}(a)}{a_{i}-a}\right]=\frac{\eta}{r+\kappa}\left[\frac{\bar{u}_{i}\left(a_{i}\right)-\bar{u}_{i}(a)-\bar{u}_{i}^{\prime}(a)\left(a_{i}-a\right)}{\left(a_{i}-a\right)^{2}}\right],
$$

which strictly negative, since $\bar{u}_{i}$ is strictly concave.

Proof of Proposition 7. Let $q(\kappa, r), a_{i}(\kappa, r)$, and $\phi_{j i}(\kappa, r)$ denote, respectively, the equilib$\operatorname{rium} q, a_{i}$, and $\phi_{j i}$ that solve (18), (19), and (20) for all $i, j \in \mathbb{X}$. We proceed in three steps: (i) show that $\phi_{j i}(\kappa, r)>0$ for all $\kappa \in(0, \infty)$ and all $r \in[0, \infty)$ provided $a_{i}(\kappa, r) \neq a_{j}(\kappa, r)$ and $\eta>0 ;(i i)$ establish that $\lim _{\kappa \rightarrow \infty} \phi_{j i}(\kappa, r)=0$ for any $r \geq 0$ and all $(i, j) \in \mathbb{X}^{2}$; (iii) show that for each $\kappa \in(0, \infty)$ there is $\bar{r}>0$ such that $\phi_{j i}(0, r)<\phi_{j i}(\kappa, r)$ for all $r \in(0, \bar{r})$. The nonmonotonicity of $\phi_{j i}(\kappa, r)$ with respect to $\kappa$ for all $r \in[0, \bar{r})$ will then follow from steps $(i)$ through (iii).

(i) From (20), $\phi_{i j}=\frac{\eta}{r+\kappa}\left\{\max _{a^{\prime}}\left[\bar{u}_{i}\left(a^{\prime} ; \kappa, r\right)-q a^{\prime}\right]-\left[\bar{u}_{i}\left(a_{j} ; \kappa, r\right)-q a_{j}\right]\right\}$, so $\phi_{i j}(\kappa, r)>0$ for all $\kappa \in(0, \infty)$ and all $r \in[0, \infty)$ provided $\eta>0$ and $a_{j} \neq \arg \max _{a^{\prime} \geq 0}\left[\bar{u}_{i}\left(a^{\prime}\right)-q a^{\prime}\right]$ (i.e., provided the investor trades).

(ii) $\lim _{\kappa \rightarrow \infty} q(\kappa, r)=q^{*}$ and $\lim _{\kappa \rightarrow \infty} a_{i}(\kappa, r)=\arg \max _{a^{\prime} \geq 0}\left[u_{i}\left(a^{\prime}\right)-q^{*} a^{\prime}\right] \equiv h_{i}^{\infty}\left(q^{*}\right)$, where $q^{*}$ is independent of $r$ and solves $\sum_{i=1}^{I} \pi_{i} h_{i}^{\infty}\left(q^{*}\right)=A$, which in turn implies $q^{*} \in(0, \infty)$, $h_{i}^{\infty}\left(q^{*}\right)<\infty$, and hence $\left|u_{i}\left(a_{j}\right)-q^{*} a_{j}\right|<\infty$ for all $(i, j) \in \mathbb{X}^{2}$. Therefore $\lim _{\kappa \rightarrow \infty} \phi_{i j}(\kappa, r)=0$ for any $r \geq 0$ and all $(i, j) \in \mathbb{X}^{2}$.

(iii) Let $\kappa \rightarrow 0$ to obtain $q(0, r)=\tilde{q}(r)$ and $a_{i}(0, r)=\arg \max _{a^{\prime} \geq 0}\left[\tilde{u}_{i}\left(a^{\prime}\right)-\tilde{q} a^{\prime}\right] \equiv h_{i}^{0}(\tilde{q})$, where $\tilde{u}_{i}(a ; r)=\frac{r u_{i}(a)+\delta \tilde{u}(a)}{r+\delta}, \tilde{u}(a)=\sum_{k=1}^{I} \pi_{k} u_{k}(a)$ and $\tilde{q}$ solves $\sum_{i=1}^{I} \pi_{i} h_{i}^{0}(\tilde{q})=A$. From (20),

$$
\phi_{j i}(0, r)=\eta \frac{\left\{r\left[u_{i}\left(a_{i}\right)-u_{i}\left(a_{j}\right)\right]+\delta \sum_{k=1}^{I} \pi_{k}\left[u_{k}\left(a_{i}\right)-u_{k}\left(a_{j}\right)\right]\right\}-(r+\delta) \tilde{q}(r)\left(a_{i}-a_{j}\right)}{(r+\delta) r} .
$$


Observe that $\lim _{r \rightarrow 0} a_{i}(0, r)=\tilde{u}^{\prime-1}[\tilde{q}(0)]=A$, for each $i \in \mathbb{X}$. Totally differentiate (18) and (19) with respect to $r$ and evaluate at $\kappa=r=0$ to find $\frac{\partial a_{i}(0,0)}{\partial r}=\frac{\tilde{q}(0)+\delta \tilde{q}^{\prime}(0)-u_{i}^{\prime}(A)}{\delta \sum_{k=1}^{I} \pi_{k} u_{k}^{\prime \prime}(A)}$ and $\sum_{i=1}^{I} \pi_{i} \frac{\partial a_{i}(0,0)}{\partial r}=0$. Combine these conditions to get $\frac{\tilde{q}(0)+\delta \tilde{q}^{\prime}(0)-\tilde{u}^{\prime}(A)}{\delta \sum_{k=1}^{I} \pi_{k} u_{k}^{\prime \prime}(A)}=0$, which together with the investor's first-order condition, $\tilde{u}^{\prime}(A)=\tilde{q}(0)$, implies $\tilde{q}^{\prime}(0)=0$ and hence $\frac{\partial a_{i}(0,0)}{\partial r}=$ $\frac{\tilde{q}(0)-u_{i}^{\prime}(A)}{\delta \sum_{k=1}^{I} \pi_{k} u_{k}^{\prime \prime}(A)}$. With this, apply L'Hôpital's rule to $(35)$ to find $\lim _{r \rightarrow 0} \phi_{j i}(0, r)=0$.

Our assumptions on primitives imply that $q(\kappa, r)$ and $a_{i}(\kappa, r)$ are continuous functions, so $\phi_{j i}(\kappa, r)$ is continuous. Hence, for each $(i, j)$ with $i \neq j$ and each $\kappa \in(0, \infty)$, there is some $\bar{r}>0$ such that for all $r \in[0, \bar{r})$, we have $\lim _{\kappa \rightarrow \infty} \phi_{j i}(\kappa, r)=0<\phi_{j i}(\kappa, r)$ (by $(i)$ and $(i i)$ ) and $\phi_{j i}(0, r)<\phi_{j i}(\kappa, r)$ (by $(i)$ and $(i i i)$ ), which establishes the nonmonotonicity of $\phi_{i j}$ with respect to $\kappa$.

Proof of Corollary 1. Write $\Phi(\alpha, \eta, r)=\sum_{i, j=1}^{I} n_{j i}(\alpha) \phi_{j i}[\alpha(1-\eta), r]$, where $n_{j i}(\alpha)$ is given by $(21)$. Fix an arbitrary $(\alpha, \eta) \in(0, \infty) \times(0,1)$. From part $(i)$ of the proof of Proposition $7, \phi_{I j}[\alpha(1-\eta), r]>0$ for $j<I$ and all $r \in[0, \infty)$. Hence, $\Phi(\alpha, \eta, r)>0$ for all $\alpha(1-\eta) \in$ $(0, \infty)$ and all $r \in[0, \infty)$. Following a similar reasoning as in part (iii) of the proof of Proposition 7 , for each $(i, j) \in \mathbb{X}^{2}$, there is $\bar{r}_{j i}>0$ such that for all $r \in\left[0, \bar{r}_{j i}\right), \phi_{j i}(0, r)<\Phi(\alpha, \eta, r)$. Then $\Phi(0, \eta, r)<\Phi(\alpha, \eta, r)$ for any $r \in\left[0, r_{0}\right)$, where $r_{0}=\min _{(i, j) \in \mathbb{X}^{2}} \bar{r}_{j i}$. Finally, from part $(i i)$ of the proof of Proposition 7, for any $r \geq 0$ we have $\lim _{\alpha^{\prime} \rightarrow \infty} \Phi\left(\alpha^{\prime}, \eta, r\right)=0<\Phi(\alpha, \eta, r)$, which establishes the nonmonotonicity of $\Phi$ with respect to $\alpha$, and therefore with respect to $\kappa=\alpha(1-\eta)$.

Proof of Proposition 8. In an equilibrium with entry the measure of dealers satisfies

$$
\Phi[\alpha(v), \eta, r]=\gamma v^{1-\theta}
$$

From Corollary 1 , there is $\tilde{r}>0$ such that $\underline{\gamma} \equiv \Phi(0, \eta, r)<\sup _{v} \Phi[\alpha(v), \eta, r] \equiv \bar{\gamma}$ for all $r \in[0, \tilde{r})$, and $\lim _{v \rightarrow \infty} \Phi[\alpha(v), \eta, r]=0<\underline{\gamma}$. Note that as $\theta \rightarrow 1, \gamma v^{1-\theta}$ converges uniformly to $\gamma$ on any closed interval $\left[v_{0}, v_{1}\right] \subseteq(0, \infty)$. Thus, for any $\gamma \in(\underline{\gamma}, \bar{\gamma})$, there is a $\tilde{\theta}$ such that for for all $\theta \in(\tilde{\theta}, 1)$, there are multiple values of $v>0$ that satisfy (36). 


\section{B Generalized preference shocks}

In this appendix we generalize the stochastic process for preference shocks as follows. As before, each investor receives a preference shock with Poisson arrival rate $\delta$, and this process is independent across investors. But now we let $\Pi=\left[\pi_{i j}\right]$ denote an $I \times I$ matrix and assume that conditional on receiving a preference shock, an investor with preference type $i$ draws preference type $j$ with probability $\pi_{i j}>0$, with $\sum_{j=1}^{I} \pi_{i j}=1$ for all $i \in \mathbb{X}$. The formulation studied in the body of the paper corresponds to the i.i.d. case, $\pi_{i j}=\pi_{j}$ for all $i$.

Equilibrium. The investor's value function $V_{i}(a, t)$ still satisfies (1) and the dealer's value function is unchanged. The bargaining outcome is also unchanged, so $V_{i}(a, t)$ also satisfies (5). The following lemma generalizes Lemma 1.

Lemma 5 An investor with preference type $i$ and asset holdings a who readjusts his asset position at time $t$ solves

$$
\max _{a^{\prime} \geq 0}\left[\bar{u}_{i}\left(a^{\prime}\right)-q(t) a^{\prime}\right]
$$

where

$$
\begin{aligned}
& \bar{u}_{i}(a)=\sum_{k=0}^{\infty} \sum_{j=1}^{I} \mu_{k} \pi_{i j}^{(k)} u_{j}(a) \quad \text { for } i=1, \ldots, I, \\
& q(t)=(r+\kappa)\left[p(t)-\kappa \int_{0}^{\infty} e^{-(r+\kappa) s} p(t+s) d s\right], \\
& \Pi^{k}=\left[\pi_{i j}^{(k)}\right] \text { for } k \geq 1, \pi_{i j}^{(0)}=\mathbb{I}_{\{j=i\}}, \text { and } \mu_{k}=\left(\frac{r+\kappa}{r+\kappa+\delta}\right)\left(\frac{\delta}{r+\kappa+\delta}\right)^{k} .
\end{aligned}
$$

Proof. As before, $V_{i}(a, t)$ satisfies (27), so the problem of an investor with preference shock $i$ who gains access to the market at time $t$ is given by (29) with $\bar{U}_{i}(a)$ as in (28). Notice that (31) is unchanged, so we only have to calculate $\bar{U}_{i}(a)$. Equation $(28)$ can be written as

$$
(r+\kappa+\delta) \bar{U}_{i}(a)=u_{i}(a)+\delta \sum_{j=1}^{I} \pi_{i j} \bar{U}_{j}(a) \quad \text { for } i=1, \ldots, I,
$$

or equivalently,

$$
\left(\mathbf{I}-\frac{\delta}{r+\kappa+\delta} \Pi\right) \overline{\mathbf{u}}=\frac{r+\kappa}{r+\kappa+\delta} \mathbf{u}
$$

where $\mathbf{I}$ is the identity matrix, and $\overline{\mathbf{u}}$ and $\mathbf{u}$ are $I \times 1$ vectors with $i^{\text {th }}$ entry $\bar{u}_{i}(a) \equiv(r+\kappa) \bar{U}_{i}(a)$ and $u_{i}(a)$, respectively. Since $\lim _{k \rightarrow \infty}\left(\frac{\delta}{r+\kappa+\delta} \Pi\right)^{k}=0,\left(\mathbf{I}-\frac{\delta}{r+\kappa+\delta} \Pi\right)^{-1}$ exists, $\sum_{k=0}^{\infty}\left(\frac{\delta}{r+\kappa+\delta} \Pi\right)^{k}$ 
converges, and $\left(\mathbf{I}-\frac{\delta}{r+\kappa+\delta} \Pi\right)^{-1}=\sum_{k=0}^{\infty}\left(\frac{\delta}{r+\kappa+\delta} \Pi\right)^{k}$. Thus, $\overline{\mathbf{u}}=\sum_{k=0}^{\infty}\left(\frac{\delta}{r+\kappa+\delta} \Pi\right)^{k} \frac{r+\kappa}{r+\kappa+\delta} \mathbf{u}$ which can be written as in (38). Substitute $\bar{U}_{i}(a)=\frac{\bar{u}_{i}(a)}{r+\kappa}$ and (31) into (29) and multiply through by $(r+\kappa)$ to obtain the formulation of the investor's problem as stated in the lemma.

Intuitively, $\bar{u}_{i}(a) /(r+\kappa)$ is the expected discounted utility to an investor with preference type $i$ from holding $a$ until the next (effective) time when he readjusts his holdings. We can write

$$
\bar{u}_{i}(a)=\sum_{k=0}^{\infty} \mu_{k} \bar{u}_{i}^{(k)}(a),
$$

where $\mu_{k}$ is the probability the investor receives $k$ preference shocks before his next effective contact with a dealer, and

$$
\bar{u}_{i}^{(k)}(a) \equiv \sum_{j=1}^{I} \pi_{i j}^{(k)} u_{j}(a)
$$

is his expected utility conditional on preference type $i$ and conditional on his receiving $k$ preference shocks over that time period. With this generalized expression for $\bar{u}_{i}(a)$, a choice of asset holdings, $a_{i}(t)$, still satisfies (9), and Lemma 2 and (11) remain unchanged.

The law of motion for the measure of investors with preference type $i$ is $\dot{n}_{i}(t)=\delta \sum_{j=1}^{I} \pi_{j i} n_{j}(t)-$ $\delta n_{i}(t)$, which implies $\mathbf{n}(t)=\mathbf{n}(0) e^{\delta(\Pi-\mathbf{I}) t}$, where $\mathbf{I}$ is the $I \times I$ identity matrix and $\mathbf{n}(t)$ denotes the $1 \times I$ vector with $i^{\text {th }}$ element $n_{i}(t)$. Thus,

$$
n_{i}(t)=\sum_{j=1}^{I} \rho_{j i}(t) n_{j}(0),
$$

where $\rho_{j i}(t)$ denotes the $j i^{\text {th }}$ element of the matrix $e^{\delta(\Pi-\mathbf{I}) t}$ and represents the transition probability for an investor from preference type $j$ to preference type $i$ in a period of length $t$. The clearing condition in the interdealer market is still (13), but with $n_{i}(t)$ given by (42). With this, it is straightforward to show that Lemma 3 generalizes as follows.

Lemma 6 The measure of investors across individual states at time $t$ satisfies (14) for all $(\mathcal{A}, \mathcal{I}) \in \Sigma$, where

$$
\begin{aligned}
n_{j i}^{0}(\mathcal{A}, t) & =e^{-\alpha t} \rho_{j i}(t) H_{0}(\mathcal{A},\{j\}) \\
n_{j i}(\tau, t) & =\alpha e^{-\alpha \tau} \rho_{j i}(\tau) n_{j}(t-\tau) .
\end{aligned}
$$


An equilibrium is a time-path $\left\langle\left\{a_{i}(t)\right\}, q(t), p(t),\left\{\phi_{i}(a, t)\right\}, H_{t}\right\rangle$ that satisfies (9) (with $\bar{u}_{i}(a)$ given by (38)), (10), (11), (13) (with $n_{i}(t)$ given by (42)) and (14) (with $n_{j i}^{0}(\mathcal{A}, t)$ and $n_{j i}(\tau, t)$ by (43) and (44), respectively). The proof of Proposition 1 can be immediately extended to show that there exists a unique equilibrium. In the limiting case $\alpha \rightarrow \infty$, we have $\bar{u}_{i}(a) \rightarrow u_{i}(a)$ (from (40)) and $u_{i}^{\prime}\left[a_{i}(t)\right] \leq q(t)=\operatorname{rp}(t)-\dot{p}(t)$ for all $i$ (from (8) and (9)). Also, $q(t) \rightarrow q^{*}(t)$, where $q^{*}(t)$ solves $\sum_{i \in \mathcal{I}_{t}^{+}} n_{i}(t) u_{i}^{\prime-1}\left[q^{*}(t)\right]=A$ and $\mathcal{I}_{t}^{+}=\left\{i \in \mathbb{X}: a_{i}(t)>0\right\}$ (from (13)), and $\phi_{i}(a, t) \rightarrow 0$ for all $a, i$ and $t$ (from (11)). Finally, $\alpha \rightarrow \infty$ implies that every investor holds his desired asset position at all times. Thus, as before, the equilibrium fees, asset price, and distribution of asset holdings converge to their Walrasian counterparts as frictions vanish.

Efficiency. Proposition 2 generalizes as follows. The planner's problem is

$$
\max _{\left\{a_{i}(t)\right\}} \int_{0}^{\infty} \frac{\alpha}{r+\alpha} \sum_{i=1}^{I} \hat{u}_{i}\left[a_{i}(t)\right] n_{i}(t) e^{-r t} d t
$$

subject to $\sum_{i=1}^{I} n_{i}(t) a_{i}(t) \leq A$, where $n_{i}(t)$ is given by $(42)$ and $\hat{u}_{i}(a)=\sum_{k=0}^{\infty} \hat{\mu}_{k} \bar{u}_{i}^{(k)}$, with $\hat{\mu}_{k}=$ $\left(\frac{r+\alpha}{r+\alpha+\delta}\right)\left(\frac{\delta}{r+\alpha+\delta}\right)^{k}$. The first-order necessary and sufficient conditions are: $(a) \hat{u}_{i}^{\prime}\left[a_{i}(t)\right] \leq \lambda(t)$ for $i=1, \ldots, I$ (with "=" if $a_{i}(t)>0$ ), where $\lambda(t)$ is the multiplier on the resource constraint, and $(b) \sum_{i=1}^{I} n_{i}(t) a_{i}^{*}[\lambda(t)]=A$, where $a_{i}^{*}[\lambda(t)]$ is the $a_{i}(t)$ that satisfies $(a)$. Notice that $\hat{\mu}_{k}=\mu_{k}$, and hence $\hat{u}_{i}=\bar{u}_{i}$ if and only if $\eta=0$. Hence, if we set $q(t)=\lambda(t)$ we find that the competitive allocation $\left\{a_{i}(t)\right\}$ coincides with the efficient allocation $\left\{a_{i}^{*}(t)\right\}$ if and only if $\eta=0$.

Steady state. Our assumptions ensure that there exists a unique row vector $\pi^{*}=\left[\pi_{i}^{*}\right]$ such that $\pi^{*}(\Pi-\mathbf{I})=\mathbf{0}$ with $\sum_{i=1}^{I} \pi_{i}^{*}=1$, and that $\lim _{t \rightarrow \infty} \rho_{j i}(t)=\pi_{i}^{*}$. Hence, (42) implies $\lim _{t \rightarrow \infty} n_{i}(t)=\pi_{i}^{*}$ for all $i$. The generalization of Proposition 3 is straightforward. The equilibrium allocations and prices $\left\langle\left\{a_{i}(t)\right\}, q(t), p(t),\left\{\phi_{i}(a, t)\right\}, H_{t}\right\rangle$ converge to the unique steadystate allocations and prices $\left\langle\left\{a_{i}\right\}, q, p,\left\{\phi_{i}(a)\right\}, H\right\rangle$ that satisfy $p=q / r, \bar{u}_{i}^{\prime}\left(a_{i}\right) \leq q$ ("=" if $a_{i}>0$, with $\bar{u}_{i}$ as in (38)), $\sum_{i=1}^{I} \pi_{i}^{*} a_{i}=A, \phi_{i}(a)$ as in $(20)$, and $\lim _{t \rightarrow \infty} H_{t}(\mathcal{A}, \mathcal{I})=H(\mathcal{A}, \mathcal{I})$, where $H\left(\left\{a_{j}\right\},\{i\}\right)=\pi_{j}^{*} \int_{0}^{\infty} \alpha e^{-\alpha \tau} \rho_{j i}(\tau) d \tau$ and $H(\mathcal{A}, \mathcal{I})=0$ for all $(\mathcal{A}, \mathcal{I}) \in \Sigma$ such that $\bigcup_{j=1}^{I}\left\{a_{j}\right\} \cap \mathcal{A}=\varnothing$.

Asset positions, prices, and trade volume. Focus on the steady state and assume $u_{i}^{\prime}(0)=$ 
$\infty$ and $u_{i}^{\prime}(\infty)=0$ for each $i$. An investor's asset choice satisfies

$$
\sum_{k=0}^{\infty} \mu_{k} \bar{u}_{i}^{(k) \prime}\left(a_{i}\right)=r p .
$$

As before, when an investor with preference type $i$ chooses his asset holdings, he evaluates his expected marginal utility from holding the asset until the next trading time. If he is hit by $k$ preference shocks over the holding period, his expected marginal utility from $a_{i}$ is $\bar{u}_{i}^{(k) \prime}\left(a_{i}\right)$. Since the number of preference shocks he experiences is random, the investor also takes expectations over $\bar{u}_{i}^{(k) \prime}\left(a_{i}\right)$ using the (discounting-adjusted) probability distribution of preference shocks, $\left\{\mu_{k}\right\}_{k=0}^{\infty}$.

Let $a_{i}=g_{i}(\kappa ; p)$ denote the choice of asset holdings characterized by (45). Then

$$
\frac{\partial g_{i}(\kappa ; p)}{\partial \kappa}=\frac{\sum_{k=0}^{\infty}\left(\frac{\delta}{r+\kappa}-k\right) \mu_{k} \bar{u}_{i}^{(k) \prime}\left(a_{i}\right)}{-\bar{u}_{i}^{\prime \prime}\left(a_{i}\right)(r+\kappa+\delta)},
$$

which generalizes (23), has the sign of the numerator. From (45), notice that $\kappa$ only affects the probability distribution $\left\{\mu_{k}\right\}$; intuitively, a marginal increase in $\kappa$ increases the probability of $k$ preference shocks for $k<\frac{\delta}{r+\kappa}$ and decreases it for $k>\frac{\delta}{r+\kappa}$. This means that an increase in $\kappa$ induces the investor to put more weight on $\bar{u}_{i}^{(k)}$ 's with smaller $k$. If shocks are i.i.d. as in the body of the paper (i.e., $\pi_{i j}=\pi_{j}$ for all $i$ ), then $\bar{u}_{i}^{(0) \prime}\left(a_{i}\right)=u_{i}^{\prime}\left(a_{i}\right)$ and $\bar{u}_{i}^{(k) \prime}\left(a_{i}\right)=\sum_{j=1}^{I} \pi_{j} u_{j}^{\prime}\left(a_{i}\right)$ for all $k \geq 1$, so in terms of preference shocks over the holding period, there are just two relevant events: either none hit or at least one hits. An increase in $\kappa$ raises the probability of the former and reduces the probability of the latter, so it makes an investor with preference type $i$ choose a larger asset position if and only if $u_{i}^{\prime}\left(a_{i}\right)>\sum_{j=1}^{I} \pi_{j} u_{j}^{\prime}\left(a_{i}\right)$. Analogously, according to (46), in this more general formulation an investor with preference type $i$ increases his asset demand in response to an increase in $\kappa$ if and only if $u_{i}^{\prime}\left(a_{i}\right)>\sum_{k=1}^{\infty}\left(\frac{\delta}{r+\kappa}-k\right) \mu_{k-1} \bar{u}_{i}^{(k) \prime}\left(a_{i}\right)$. Since this condition may seem intricate, we provide simpler conditions for some special cases.

Proposition 9 (i) Suppose the sequence $\left\{\bar{u}_{i}^{(k) \prime}\left(a_{i}\right)\right\}_{k=0}^{\infty}$ is monotone in $k$. Then $\frac{\partial g_{i}(\kappa ; p)}{\partial \kappa}>0$ if and only if

$$
u_{i}^{\prime}\left(a_{i}\right)>\sum_{j=1}^{I} \pi_{j}^{*} u_{j}^{\prime}\left(a_{i}\right) .
$$

(ii) Consider the frictionless limit, $\kappa \rightarrow \infty$. Then $\frac{\partial g_{i}(\kappa ; p)}{\partial\left(\frac{1}{r+\kappa}\right)}>0$ if and only if

$$
u_{i}^{\prime}\left(a_{i}\right)<\sum_{j=1}^{I} \pi_{i j} u_{j}^{\prime}\left(a_{i}\right) .
$$


(iii) Consider the case $I=2$. Then for $i, j \in\{1,2\}$ (with $j \neq i$ ),

$$
\bar{u}_{i}(a)=\frac{r+\kappa+\delta \pi_{j i}}{r+\kappa+\delta\left(\pi_{12}+\pi_{21}\right)} u_{i}(a)+\frac{\delta \pi_{i j}}{r+\kappa+\delta\left(\pi_{12}+\pi_{21}\right)} u_{j}(a)
$$

and $\frac{\partial g_{i}(\kappa ; p)}{\partial \kappa}>0$ if and only if $u_{i}^{\prime}\left(a_{i}\right)>u_{j}^{\prime}\left(a_{i}\right)$.

Proof. (i) From (46), $\frac{\partial g_{i}(\kappa ; p)}{\partial \kappa}$ has the sign of $\sum_{k=0}^{\infty}\left(\frac{\delta}{r+\kappa}-k\right) \mu_{k} \bar{u}_{i}^{(k) \prime}\left(a_{i}\right)$, so we sign the latter. Let $\overline{\mathbb{Z}}=\mathbb{Z} \cap\left(-\infty, \frac{\delta}{r+\kappa}\right)$, where $\mathbb{Z}$ denotes the set of integers, and define $\bar{k}=\max _{k \in \overline{\mathbb{Z}}} k$. Suppose that (47) holds. Then $\left\{\bar{u}_{i}^{(k) \prime}\right\}_{k=0}^{\infty}$ is a decreasing sequence with $\bar{u}_{i}^{(0) \prime}\left(a_{i}\right)=u_{i}^{\prime}\left(a_{i}\right)>$ $\sum_{j=1}^{I} \pi_{j}^{*} u_{j}^{\prime}\left(a_{i}\right)=\lim _{k \rightarrow \infty} \bar{u}_{i}^{(k) \prime}\left(a_{i}\right)$. Since $\left(\frac{\delta}{r+\kappa}-k\right) \mu_{k}>0$ for $k<\bar{k}+1$ and $\left(\frac{\delta}{r+\kappa}-k\right) \mu_{k} \leq 0$ for $k \geq \bar{k}+1$, the fact that $\bar{u}_{i}^{(k+1) \prime} \leq \bar{u}_{i}^{(k) \prime}$ for all $k$ implies

$$
\sum_{k=0}^{\bar{k}}\left(\frac{\delta}{r+\kappa}-k\right) \mu_{k} \bar{u}_{i}^{(\bar{k}) \prime}\left(a_{i}\right)+\sum_{k=\bar{k}+1}^{\infty}\left(\frac{\delta}{r+\kappa}-k\right) \mu_{k} \bar{u}_{i}^{(\bar{k}+1) \prime}\left(a_{i}\right) \leq \sum_{k=0}^{\infty}\left(\frac{\delta}{r+\kappa}-k\right) \mu_{k} \bar{u}_{i}^{(k) \prime}\left(a_{i}\right) .
$$

Since $\sum_{k=0}^{\infty}\left(\frac{\delta}{r+\kappa}-k\right) \mu_{k}=0$, the above inequality can be written as

$$
0 \leq\left[\bar{u}_{i}^{(\bar{k}) \prime}\left(a_{i}\right)-\bar{u}_{i}^{(\bar{k}+1) \prime}\left(a_{i}\right)\right] \sum_{k=0}^{\bar{k}}\left(\frac{\delta}{r+\kappa}-k\right) \mu_{k} \leq \sum_{k=0}^{\infty}\left(\frac{\delta}{r+\kappa}-k\right) \mu_{k} \bar{u}_{i}^{(k) \prime}\left(a_{i}\right) .
$$

If $\bar{u}_{i}^{(\bar{k}+1) \prime}\left(a_{i}\right)<\bar{u}_{i}^{(\bar{k}) \prime}\left(a_{i}\right)$, then the first inequality in (50) is strict. Alternatively, if $\bar{u}_{i}^{(\bar{k}+1) \prime}\left(a_{i}\right)=$ $\bar{u}_{i}^{(\bar{k}) \prime}\left(a_{i}\right)$ then the second inequality is strict, since $\bar{u}_{i}^{(0) \prime}\left(a_{i}\right)>\lim _{k \rightarrow \infty} \bar{u}_{i}^{(k) \prime}\left(a_{i}\right)$, which implies that $\sum_{k=0}^{\bar{k}}\left(\frac{\delta}{r+\kappa}-k\right) \mu_{k} \bar{u}_{i}^{(\bar{k}) \prime}\left(a_{i}\right)<\sum_{k=0}^{\bar{k}}\left(\frac{\delta}{r+\kappa}-k\right) \mu_{k} \bar{u}_{i}^{(k) \prime}\left(a_{i}\right)$ or $\sum_{k=\bar{k}+1}^{\infty}\left(\frac{\delta}{r+\kappa}-k\right) \mu_{k} \bar{u}_{i}^{(\bar{k}+1) \prime}\left(a_{i}\right)<$ $\sum_{k=\bar{k}+1}^{\infty}\left(\frac{\delta}{r+\kappa}-k\right) \mu_{k} \bar{u}_{i}^{(k) \prime}\left(a_{i}\right)$ must hold. In any case, $\frac{\partial g_{i}(\kappa ; p)}{\partial \kappa}>0$ follows. Conversely, suppose that $\sum_{k=0}^{\infty}\left(\frac{\delta}{r+\kappa}-k\right) \mu_{k} \bar{u}_{i}^{(k) \prime}\left(a_{i}\right)>0$, but (47) does not hold, i.e., $u_{i}^{\prime}\left(a_{i}\right) \leq \sum_{j=1}^{I} \pi_{j}^{*} u_{j}^{\prime}\left(a_{i}\right)$. Then $\left\{\bar{u}_{i}^{(k) \prime}\right\}_{k=0}^{\infty}$ is an increasing sequence, and

$$
\sum_{k=0}^{\infty}\left(\frac{\delta}{r+\kappa}-k\right) \mu_{k} \bar{u}_{i}^{(k) \prime}\left(a_{i}\right) \leq \sum_{k=0}^{\bar{k}}\left(\frac{\delta}{r+\kappa}-k\right) \mu_{k} \bar{u}_{i}^{(\bar{k}) \prime}\left(a_{i}\right)+\sum_{k=\bar{k}+1}^{\infty}\left(\frac{\delta}{r+\kappa}-k\right) \mu_{k} \bar{u}_{i}^{(\bar{k}+1) \prime}\left(a_{i}\right) .
$$

This leads to

$$
\sum_{k=0}^{\infty}\left(\frac{\delta}{r+\kappa}-k\right) \mu_{k} \bar{u}_{i}^{(k) \prime}\left(a_{i}\right) \leq\left[\bar{u}_{i}^{(\bar{k}) \prime}\left(a_{i}\right)-\bar{u}_{i}^{(\bar{k}+1) \prime}\left(a_{i}\right)\right] \sum_{k=0}^{\bar{k}}\left(\frac{\delta}{r+\kappa}-k\right) \mu_{k} \leq 0,
$$

a contradiction.

(ii) Let $\varkappa=(r+\kappa)^{-1}$ and differentiate (45) with respect to $\varkappa$ (with $p$ given) to find

$$
\frac{\partial g_{i}\left(\frac{1}{\varkappa}-r ; p\right)}{\partial \varkappa}=\frac{\frac{1}{(1+\delta \varkappa)} \sum_{k=0}^{\infty}(k-\delta \varkappa)\left(\frac{1}{1+\delta \varkappa}\right)\left(\frac{\delta \varkappa}{1+\delta \varkappa}\right)^{k} \bar{u}_{i}^{(k) \prime}\left(a_{i}\right)}{-\bar{u}_{i}^{\prime \prime}\left(a_{i}\right)} \text {. }
$$


The numerator can be written as

$$
\delta\left(\frac{1}{1+\delta \varkappa}\right)^{2}\left[\frac{1-\delta \varkappa}{1+\delta \varkappa} \bar{u}_{i}^{(1) \prime}\left(a_{i}\right)-\bar{u}_{i}^{(0) \prime}\left(a_{i}\right)+O(\varkappa)\right],
$$

where $O(\varkappa)=\sum_{k=2}^{\infty}(k-\delta \varkappa)\left(\frac{1}{1+\delta \varkappa}\right)^{k}(\delta \varkappa)^{k-1} \bar{u}_{i}^{(k) \prime}\left(a_{i}\right)$. Since $\lim _{\varkappa \rightarrow 0} O(\varkappa)=0$, we have

$$
\lim _{\varkappa \rightarrow 0} \frac{\partial g_{i}\left(\frac{1}{\varkappa}-r ; p\right)}{\partial \varkappa}=\frac{\delta\left[\bar{u}_{i}^{(1) \prime}\left(a_{i}\right)-\bar{u}_{i}^{(0) \prime}\left(a_{i}\right)\right]}{-\bar{u}_{i}^{\prime \prime}\left(a_{i}\right)} .
$$

Finally, $\bar{u}_{i}^{(0) \prime}\left(a_{i}\right)=u_{i}^{\prime}\left(a_{i}\right)$ and $\bar{u}_{i}^{(1) \prime}\left(a_{i}\right)=\sum_{j=1}^{I} \pi_{i j} u_{j}^{\prime}\left(a_{i}\right)$ imply that $\lim _{\kappa \rightarrow \infty} \frac{\partial g_{i}(\kappa ; p)}{\partial\left(\frac{1}{r+\kappa}\right)}>0$ if and only if (48) holds.

(iii) Let $I=2$. For $i=1,(38)$ reduces to

$$
\bar{u}_{1}(a)=\left(\frac{r+\kappa}{r+\kappa+\delta}\right) \sum_{k=0}^{\infty}\left(\frac{\delta}{r+\kappa+\delta}\right)^{k}\left[\pi_{11}^{(k)} u_{1}(a)+\left(1-\pi_{11}^{(k)}\right) u_{2}(a)\right],
$$

where $\pi_{11}^{(k)}=\frac{\pi_{21}}{\pi_{12}+\pi_{21}}+\frac{\pi_{12}}{\pi_{12}+\pi_{21}}\left(1-\pi_{12}-\pi_{21}\right)^{k}$, since $\pi_{12}+\pi_{21}>0$. Collect terms to arrive at (49) for $i=1$. The expression for $i=2$ is obtained similarly. The first-order condition (45) specializes to

$$
\frac{r+\kappa+\delta \pi_{j i}}{r+\kappa+\delta\left(\pi_{12}+\pi_{21}\right)} u_{i}^{\prime}\left(a_{i}\right)+\frac{\delta \pi_{i j}}{r+\kappa+\delta\left(\pi_{12}+\pi_{21}\right)} u_{j}^{\prime}\left(a_{i}\right)=r p
$$

This can be differentiated with respect to $\kappa$ (for fixed $p$ ) to obtain

$$
\frac{\partial g_{i}(\kappa ; p)}{\partial \kappa}=\frac{\delta \pi_{i j}\left[u_{i}^{\prime}\left(a_{i}\right)-u_{j}^{\prime}\left(a_{i}\right)\right]}{-\bar{u}_{i}^{\prime \prime}\left(a_{i}\right)\left[r+\kappa+\delta\left(\pi_{12}+\pi_{21}\right)\right]^{2}} .
$$

This concludes the proof.

For the i.i.d. case analyzed in the body of the paper, we found that if trading frictions decrease, an investor increases his asset holdings if his current marginal valuation exceeds his expected marginal valuation over the expected holding period (condition (23)). Proposition 9 extends this result and shows that the key insight does not rely on the preference shocks being i.i.d. For the case of multiplicative preference shocks we analyzed in Section 4, for example, we have $\bar{u}_{i}(a)=\bar{\varepsilon}_{i} u(a)$, with

$$
\bar{\varepsilon}_{i}=\sum_{k=0}^{\infty} \mu_{k} \bar{\varepsilon}_{i}^{(k)}
$$

and $\bar{\varepsilon}_{i}^{(k)}=\sum_{j=1}^{I} \pi_{i j}^{(k)} \varepsilon_{j}$. Note that $\lim _{k \rightarrow \infty} \bar{\varepsilon}_{i}^{(k)}=\sum_{j=1}^{I} \pi_{j}^{*} \varepsilon_{j} \equiv \bar{\varepsilon}$. Part $(i)$ of Proposition 9 establishes that if this convergence is monotonic for $i$, then an investor with preference type 
$i$ increases his asset holdings if and only if $\varepsilon_{i}>\bar{\varepsilon}$. This is essentially the same condition we derived in the i.i.d. case where $\pi_{i j}^{(k)}=\pi_{j}^{*}$ for all $i$ and all $k \geq 1$. For this multiplicative, case the condition in part $(i i)$ of the proposition reduces to $\varepsilon_{i}>\bar{\varepsilon}_{i}^{(1)}$, and if we let $\delta_{i j} \equiv \delta \pi_{i j}$ for $i \neq j$ and $\delta_{i i} \equiv \delta\left(1-\pi_{i i}\right)$, it can be written as

$$
\varepsilon_{i}>\frac{\sum_{j \neq i} \delta_{i j} \varepsilon_{j}}{\sum_{j \neq i} \delta_{i j}}
$$

Proposition 9 parallels Proposition 2 in Gârleanu (2006). Notation aside, (52) is identical to the condition in part $(i)$ of his Proposition 2. The monotonicity condition in part $(i i)$ of his proposition plays the role of the monotonicity condition in part $(i)$ of ours. The two-valuation case in part ( $i i i)$ of his proposition parallels part ( $i i i)$ in ours.

An implication of the i.i.d. case that does not generalize is that if $\varepsilon_{i}<\varepsilon_{j}$ and the agent with preference type $i$ increases his asset holdings in response to an increase in $\kappa$, then so does the agent with preference type $j .{ }^{23}$ The robust insight instead is that an investor whose current marginal valuation is large - in the sense that it exceeds his expected marginal valuation over the expected holding period - increases his asset holdings if $\kappa$ increases.

The following proposition characterizes the equilibrium price for a particular class of utility functions and generalizes the discussion that followed Proposition 4. Just as in the i.i.d. case, this price is independent of frictions as summarized by $\kappa$ if the individual asset demand is linear in the idiosyncratic valuation (as is the case with logarithmic preferences).

Proposition 10 Let $u_{i}(a)=\varepsilon_{i} a^{1-\sigma} /(1-\sigma)$ with $\sigma>0$. Then

$$
p=\frac{\left(\sum_{i=1}^{I} \pi_{i}^{*} \bar{\varepsilon}_{i}^{1 / \sigma}\right)^{\sigma}}{r A^{\sigma}}
$$

where $\bar{\varepsilon}_{i}=\sum_{k=0}^{\infty} \sum_{j=1}^{I} \mu_{k} \pi_{i j}^{(k)} \varepsilon_{j}$. If $u_{i}(a)=\varepsilon_{i} \ln a$, then

$$
p=\frac{\sum_{j=1}^{I} \pi_{j}^{*} \varepsilon_{j}}{r A} .
$$

Proof. Since $u_{i}(a)=\varepsilon_{i} u(a)$, we have $\bar{u}_{i}(a)=\bar{\varepsilon}_{i} u(a)$ with $\bar{\varepsilon}_{i}$ given by (51), so (45) becomes $\bar{\varepsilon}_{i} u^{\prime}\left(a_{i}\right)=r p$. The parametric assumption implies $a_{i}=\left(\bar{\varepsilon}_{i} /(r p)\right)^{1 / \sigma}$ so the steadystate market-clearing condition, $\sum_{i=1}^{I} \pi_{i}^{*} a_{i}=A$, yields the first expression for $p$. For $\sigma=1$, $p=(r A)^{-1} \sum_{i=1}^{I} \pi_{i}^{*} \bar{\varepsilon}_{i}$, where $\sum_{i=1}^{I} \pi_{i}^{*} \bar{\varepsilon}_{i}=\sum_{j=1}^{I} \sum_{k=0}^{\infty} \sum_{i=1}^{I} \pi_{i}^{*} \pi_{i j}^{(k)} \mu_{k} \varepsilon_{j}=\sum_{j=1}^{I} \pi_{j}^{*} \varepsilon_{j}$.

\footnotetext{
${ }^{23}$ For example, with a more general process for preference shocks it is possible to have a parametrization $\left\{\varepsilon_{i}, \pi_{i j}\right\}_{i, j=1}^{I}$ with $\sum_{k=1}^{I} \pi_{i k} \varepsilon_{k}<\varepsilon_{i}<\varepsilon_{j}<\sum_{k=1}^{I} \pi_{j k} \varepsilon_{k}$, which according to part (ii) of Proposition 9 implies that, near the frictionless limit, the high valuation investor (the one with preference type $\varepsilon_{j}$ ) will reduce his asset holdings and the low valuation investor will increase his asset holdings if $\kappa$ increases.
} 
As in the i.i.d. case, it is difficult to sign the general equilibrium effects of $\alpha$ and $\eta$ on trade volume in general. We provide analytical results for three cases. The first has $I=2$ and a general preference specification, the second considers a market close to the frictionless limit, and the third a market with severe trading frictions.

Proposition 11 (i) Let $u_{i}(a)=\varepsilon_{i} a^{1-\sigma} /(1-\sigma)$ with $\sigma>0$, and assume that $I=2$. Trade volume increases with $\kappa$.

(ii) Let $u_{i}(a)=\varepsilon_{i} \ln a$ and suppose that $\bar{\varepsilon}_{j}>\bar{\varepsilon}_{i}$ implies $\varepsilon_{j}-\sum_{k=1}^{I} \pi_{j k} \varepsilon_{k}>\varepsilon_{i}-\sum_{k=1}^{I} \pi_{i k} \varepsilon_{k}$ for all $i, j \in \mathbb{X}^{2}$. Trade volume decreases with $\eta$ in the frictionless limit (as $\left.\kappa \rightarrow \infty\right)$.

(iii) Trade volume approaches zero as $r+\kappa \rightarrow 0$.

Proof. $(i)$ With $I=2, n_{12}=n_{21}=\frac{\delta \pi_{12} \pi_{21}}{\left[\alpha+\delta\left(\pi_{12}+\pi_{21}\right)\right]\left(\pi_{12}+\pi_{21}\right)}$, so trade volume is

$$
\mathcal{V}=\frac{\alpha \delta \pi_{12} \pi_{21}}{\left[\alpha+\delta\left(\pi_{12}+\pi_{21}\right)\right]\left(\pi_{12}+\pi_{21}\right)}\left(a_{2}-a_{1}\right)
$$

The preference specification together with (49) implies $a_{i}=\left(\bar{\varepsilon}_{i} /(r p)\right)^{1 / \sigma}$ for $i=1,2$, where $\bar{\varepsilon}_{1}=\frac{r+\kappa+\delta \pi_{21}}{r+\kappa+\delta\left(\pi_{12}+\pi_{21}\right)} \varepsilon_{1}+\frac{\delta \pi_{12}}{r+\kappa+\delta\left(\pi_{12}+\pi_{21}\right)} \varepsilon_{2}$ and $\bar{\varepsilon}_{2}=\frac{r+\kappa+\delta \pi_{12}}{r+\kappa+\delta\left(\pi_{12}+\pi_{21}\right)} \varepsilon_{2}+\frac{\delta \pi_{21}}{r+\kappa+\delta\left(\pi_{12}+\pi_{21}\right)} \varepsilon_{1}$. Since $r p=\frac{\left(\pi_{1}^{*} \bar{\varepsilon}_{1}^{1 / \sigma}+\pi_{2}^{*} \bar{\varepsilon}_{2}^{1 / \sigma}\right)^{\sigma}}{A^{\sigma}}$,

$$
a_{i}=\frac{\bar{\varepsilon}_{i}^{1 / \sigma}}{\pi_{1}^{*} \bar{\varepsilon}_{1}^{1 / \sigma}+\pi_{2}^{*} \bar{\varepsilon}_{2}^{1 / \sigma}} A .
$$

Differentiate this expression with respect to $\kappa$ to find that $\frac{\partial a_{2}}{\partial \kappa}$ has the sign of $\left(\varepsilon_{2}-\varepsilon_{1}\right)$ and $\frac{\partial a_{1}}{\partial \kappa}$ has the opposite sign. Since $\varepsilon_{1}<\varepsilon_{2}$, $\frac{d a_{1}}{d \kappa}<0<\frac{d a_{2}}{d \kappa}$. To find $\frac{d \mathcal{V}}{d \kappa}$, we consider two cases. (a) An increase in $\kappa$ caused by a decrease in $\eta$ (keeping $\alpha$ constant). For this case, $\frac{d \mathcal{V}}{d \kappa}=\frac{d a_{2}}{d \kappa}-\frac{d a_{1}}{d \kappa}>0$. (b) An increase in $\kappa$ caused by an increase in $\alpha$, which implies $\frac{d \mathcal{V}}{d \kappa}=$ $\left[\frac{\delta}{\alpha+\delta\left(\pi_{12}+\pi_{21}\right)}\right]^{2} \pi_{12} \pi_{21}\left(a_{2}-a_{1}\right)+\frac{\alpha \delta \pi_{12} \pi_{21}}{\left[\alpha+\delta\left(\pi_{12}+\pi_{21}\right)\right]\left(\pi_{12}+\pi_{21}\right)}\left(\frac{d a_{2}}{d \kappa}-\frac{d a_{1}}{d \kappa}\right)>0$.

(ii) Let $\varkappa=(r+\kappa)^{-1}$. Under $u_{i}(a)=\varepsilon_{i} \ln a$, (45) implies $a_{i}=\bar{\varepsilon}_{i} /(r p)$, where $\bar{\varepsilon}_{i}=$ $\sum_{k=0}^{\infty} \mu_{k} \bar{\varepsilon}_{i}^{(k)}$, with $\bar{\varepsilon}_{i}^{(k)}=\sum_{j=1}^{I} \pi_{i j}^{(k)} \varepsilon_{j}$ and $\mu_{k}=\left(\frac{1}{1+\delta \varkappa}\right)\left(\frac{\delta \varkappa}{1+\delta \varkappa}\right)^{k}$. Differentiate with respect to $\varkappa$ to find

$$
\frac{d a_{i}}{d \varkappa}=\frac{1}{(1+\delta \varkappa) \varkappa} \sum_{k=0}^{\infty}(k-\delta \varkappa) \mu_{k} \bar{\varepsilon}_{i}^{(k)} .
$$

We know from Proposition 10 that under this preference specification the equilibrium price is independent of $\kappa$, so (53) captures the general equilibrium effect of $\kappa$ on $a_{i}$. Let $\varkappa \rightarrow 0$ as in part $(i i)$ of the proof of Proposition 9 to find

$$
\lim _{\varkappa \rightarrow 0} \frac{d a_{i}}{d \varkappa}=\frac{\delta\left[\bar{\varepsilon}_{i}^{(1)}-\bar{\varepsilon}_{i}^{(0)}\right]}{r p} .
$$


Therefore,

$$
\frac{d\left(a_{j}-a_{i}\right)}{d \varkappa}=\frac{\delta}{r p}\left\{\bar{\varepsilon}_{j}^{(1)}-\varepsilon_{j}-\left[\bar{\varepsilon}_{i}^{(1)}-\varepsilon_{i}\right]\right\}
$$

The assumption that $\varepsilon_{j}-\bar{\varepsilon}_{j}^{(1)}>\varepsilon_{i}-\bar{\varepsilon}_{i}^{(1)}$ if $\bar{\varepsilon}_{j}>\bar{\varepsilon}_{i}$ implies $\frac{d\left(a_{j}-a_{i}\right)}{d \varkappa}<0$ for $a_{j}>a_{i}$ and $\frac{d\left(a_{j}-a_{i}\right)}{d \varkappa}>0$ for $a_{j}<a_{i}$, so an increase in $\varkappa$ decreases the size of every trade. If the increase in $\varkappa$ is due to an increase in $\eta$, i.e., keeping $\alpha$ constant, then the weights $n_{i j}$ in (24) remain constant and $\mathcal{V}$ decreases.

(iii) From (38), $\bar{u}_{i}(a)=\sum_{j=1}^{I} \omega_{i j}(\bar{r}) u_{j}(a)$ where $\omega_{i j}(\bar{r})=\sum_{k=0}^{\infty}\left(\frac{\bar{r}}{\bar{r}+\delta}\right)\left(\frac{\delta}{\bar{r}+\delta}\right)^{k} \pi_{i j}^{(k)}$ and $\bar{r}=$ $r+\kappa$. We first show that for any $\varepsilon>0,\left|\omega_{i j}(\bar{r})-\pi_{j}^{*}\right|<\varepsilon$ obtains for all $\bar{r}$ close enough to 0 . For any $\bar{r}>0$ and any $N \in \mathbb{Z}_{+}$,

$$
\left|\omega_{i j}(\bar{r})-\pi_{j}^{*}\right| \leq\left|\sum_{k=0}^{N}\left(\frac{\bar{r}}{\bar{r}+\delta}\right)\left(\frac{\delta}{\bar{r}+\delta}\right)^{k}\left[\pi_{i j}^{(k)}-\pi_{j}^{*}\right]\right|+\left|\sum_{k=N+1}^{\infty}\left(\frac{\bar{r}}{\bar{r}+\delta}\right)\left(\frac{\delta}{\bar{r}+\delta}\right)^{k}\left[\pi_{i j}^{(k)}-\pi_{j}^{*}\right]\right| .
$$

Since $\lim _{k \rightarrow \infty} \pi_{i j}^{(k)}=\pi_{j}^{*}$, choose $N$ large enough so that the second term is strictly smaller than $\varepsilon / 2$ for any $\bar{r}>0$. The first term is bounded above by $\left|1-\left(\frac{\delta}{\bar{r}+\delta}\right)^{N+1}\right|$, so it is strictly less than $\varepsilon / 2$ for all $\bar{r}$ close enough to 0 . Therefore, $\lim _{\bar{r} \rightarrow 0} \omega_{i j}(\bar{r})=\pi_{j}^{*}$, and $\lim _{\bar{r} \rightarrow 0} \bar{u}_{i}(a)=\sum_{j=1}^{I} \pi_{j}^{*} u_{j}(a)$ for every $i$. In turn, (37) approaches $\max _{a^{\prime} \geq 0}\left[\sum_{j=1}^{I} \pi_{j}^{*} u_{j}\left(a^{\prime}\right)-q(t) a^{\prime}\right]$, so $a_{i} \rightarrow A$ for all $i$. With this, $\mathcal{V} \rightarrow 0$ as $r+\kappa \rightarrow 0$ is immediate from (24).

Part $(i)$ of Proposition 11 is a generalization of part ( $i$ ) of Proposition 6. Part (ii) of Proposition 11 is analogous to part ( $i i)$ of Proposition 6. The focus of the former on the frictionless limit simplifies the analysis of the effects of trading frictions on individual asset demands (see, e.g., part (ii) of Proposition 9). The additional assumption is a condition on the speed with which preference shocks revert to their unconditional mean. For example, suppose $\bar{\varepsilon}_{j}>\bar{\varepsilon}_{i}$, which means that the expected marginal valuation over the holding period for an investor who currently has preference type $j$ is larger than for an investor with preference type $i$. Then the assumption requires that the expected change in the marginal valuation after a single preference shock (e.g., $\varepsilon_{j}-\sum_{k=1}^{I} \pi_{j k} \varepsilon_{k}$ for the agent with preference type $j$ ) must be larger for the investor with the higher current expected valuation over the holding period. Part (iii) of Proposition 11 generalizes part ( $i i i)$ of Proposition 6 as well as the notion - which for the i.i.d. case was proved in part ( $i i$ ) of Proposition 5 and used in the proofs of Proposition 7, Corollary 1, and Proposition 8 - that if the investor is patient, the influence of his current valuation at the time of the trade on his choice of asset holdings vanishes as the market becomes very illiquid. In other words, as 
$r+\kappa \rightarrow 0$, the distribution of asset holdings converges to a mass point at $A$ and trade volume approaches zero. This has important implications for intermediation fees and dealer revenue: both approach zero as trade sizes vanish, just as in the i.i.d. case. Note that intermediation fees and revenue also go to zero as $\kappa$ becomes large, so they are nonmonotonic functions of $\kappa$. Therefore, the nonmonotonicity results we established for i.i.d. preference shocks (Proposition 7 and Corollary 1) generalize. Finally, these nonmonotonicities can generate multiple steady-state equilibria, so Proposition 8, which we proved for the i.i.d. case, can also be generalized. 


\section{Strategic bargaining}

In the body of the paper we assumed that when an investor and a dealer trade, the new asset position of the investor, $a^{\prime}$, and the fee, $\phi$, are the solution to a Nash bargaining problem where the dealer has bargaining power $\eta \in[0,1]$ and disagreement point $W(t)$, and the investor has disagreement point $V_{i}(a, t)$. In this appendix we offer four strategic bargaining games, each with a unique subgame perfect equilibrium outcome that coincides with the solution of the axiomatic Nash bargaining problem we have adopted.

The four games we consider have the common feature that the relationship between the investor and the dealer is short-lived in that the negotiation takes place in an "instant." In the first game, a proposer is chosen at random to make a take-it-or-leave-it offer. The second is a standard bargaining game with alternating offers and a random termination of the negotiation. We take the limit of this game as the expected time horizon for the negotiation goes to zero. The third is the off-the-shelf bargaining game of Binmore, Rubinstein, and Wolinsky (1986) incorporated into a discrete-time version of our model. Here we assume that the negotiation takes place within a period but may involve an infinite number of bargaining rounds. Finally, we consider a payoff-equivalent game that can involve at most two bargaining rounds. The fact that the unique subgame perfect equilibrium outcome in each of these four games coincides with the solution of the Nash problem as formulated in Section 3, is an indication that the disagreement points we have adopted in our axiomatic formulation are the appropriate ones when the relationship between dealers and investors is essentially instantaneous, as is the case in our environment.

\section{C.1 Take-it-or-leave-it offer by a randomly chosen proposer}

Our theory is meant to model a fast-moving market where investors and dealers don't form long-lasting relationships, but rather contact each other at relatively high frequencies and must trade on the spot, instantaneously, before they part ways. With this in mind, consider the following natural and simple strategic bargaining game. Upon contact, with probability $\eta$, Nature selects the dealer to make an instantaneous take-it-or-leave-it offer, which the investor must either accept or reject on the spot. With complementary probability, Nature selects the investor to make an instantaneous take-it-or-leave-it offer, which the dealer must either accept or reject on the spot. The whole process is instantaneous, and the dealer and the investor part 
ways regardless of the outcome. ${ }^{24}$

Let $\left\langle a_{i}^{1}(t), \phi_{i}^{1}(a, t)\right\rangle$ denote the proposal that the dealer makes to an investor of type $i$ who is holding $a$ at time $t$, and $\left\langle a_{i}^{2}(t), \phi_{i}^{2}(a, t)\right\rangle$ denote the offer that the latter makes to the former. The set of offers that an investor of type $i$ who is holding asset position $a$ finds acceptable at time $t$ is

$$
\mathcal{A}_{i}^{2}(a, t)=\left\{\left(a^{\prime}, \phi\right): V_{i}\left(a^{\prime}, t\right)-p(t)\left(a^{\prime}-a\right)-\phi \geq V_{i}(a, t)\right\} .
$$

Similarly, the set of offers that a dealer finds acceptable at time $t$ is $\mathcal{A}^{1}=\left\{\left(a^{\prime}, \phi\right): \phi \geq 0\right\}$. If the dealer is selected as the proposer, he will offer

$$
\left\langle a_{i}^{1}(t), \phi_{i}^{1}(a, t)\right\rangle=\arg \max _{\left(a^{\prime}, \phi\right)} \phi \mathbb{I}_{\mathcal{A}_{i}^{2}(a, t)}\left(a^{\prime}, \phi\right),
$$

where the maximization is subject to $a^{\prime} \geq 0$, and $\mathbb{I}_{\mathcal{A}_{i}^{2}(a, t)}\left(a^{\prime}, \phi\right)$ is an indicator function that is equal to 1 if $\left(a^{\prime}, \phi\right) \in \mathcal{A}_{i}^{2}(a, t)$. It is easy to see that $a_{i}^{1}(t)=a_{i}(t)$, where $a_{i}(t)$ is as in $(3)$, and $\eta \phi_{i}^{1}(a, t)=\phi_{i}(a, t)$, where $\phi_{i}(a, t)$ is as in (4). If the investor makes the offer, he chooses

$\left\langle a_{i}^{2}(t), \phi_{i}^{2}(a, t)\right\rangle=\arg \max _{\left(a^{\prime}, \phi\right)}\left\{\left[V_{i}\left(a^{\prime}, t\right)-p(t)\left(a^{\prime}-a\right)-\phi\right] \mathbb{I}_{\mathcal{A}^{1}}\left(a^{\prime}, \phi\right)+\left[1-\mathbb{I}_{\mathcal{A}^{1}}\left(a^{\prime}, \phi\right)\right] V_{i}(a, t)\right\}$

where the maximization is subject to $a^{\prime} \geq 0$, and $\mathbb{I}_{\mathcal{A}^{1}}\left(a^{\prime}, \phi\right)$ is an indicator function that is equal to 1 if $\left(a^{\prime}, \phi\right) \in \mathcal{A}^{1}$. Hence, $a_{i}^{2}(t)=a_{i}(t)$ and $\phi_{i}^{2}(a, t)=0$. Note that regardless of who gets selected to make the offer, the outcome of the negotiation is that the investor exits the meeting with asset position $a_{i}(t)$. The transaction fee equals $\phi_{i}(a, t) / \eta$ if the dealer makes the offer and 0 if the investor makes the offer, so the expected fee (before Nature decides who will make the offer) equals $\phi_{i}(a, t)$. It is easy to check that with these equilibrium outcomes the investors' and dealers' value functions are just as in the body of the paper and all our results go through (subject to the obvious reinterpretation of $\phi_{i}(a, t)$ as an expected intermediation fee, which is inconsequential).

\section{C.2 Alternating offers in continuous time with random termination}

Consider a strategic alternating offers bargaining game similar to the one analyzed by Rubinstein (1982), but extended to allow for exogenous breakdown in negotiations as in Binmore, Rubinstein, and Wolinsky (1986). The game we analyze belongs to the class of stochastic sequential bargaining games studied by Merlo and Wilson (1995). Time is continuous, and we

\footnotetext{
${ }^{24}$ This type of bargaining procedure has been used extensively in search models of money, e.g., Burdett, Trejos, and Wright (2001), as well as in search models of the labor market, e.g., Kiyotaki and Lagos (2007).
} 
assume that negotiations between an investor and a dealer who meet at time $t$ take place at $\tau=t, t+\Delta, t+2 \Delta, \ldots$, where $\Delta>0$ is the length of the period of time between two consecutive bargaining rounds. In every bargaining round, Nature selects a player to make an offer $\left(a^{\prime}, \phi\right)$; the dealer is selected with probability $\eta$ (which we will also denote $\eta_{1}$ ) and the investor with probability $1-\eta \equiv \eta_{2}$. If the recipient of the offer accepts it, the trade is consummated instantaneously and the match is dissolved. If the recipient rejects the offer, the pair waits for a period of length $\Delta$ until the next bargaining round. During this period of time between bargaining rounds, the dealer-investor match is subject to a Poisson destruction process with arrival rate $\beta$. (This process is independent across matches and also independent of the investor's process for preference shocks.) If the match survives until the next bargaining round, Nature again selects the player who will make the next offer, and so on. To keep the analysis simple, we describe the negotiations that take place once the market has reached the long-run steady state described in Proposition 3.

Consider an investor with asset holdings $a$ who enters a negotiation round with a dealer. The payoff to the dealer if an agreement $\left(a^{\prime}, \phi\right)$ is reached at time $\tau \geq t$ is $e^{-r(\tau-t)} \mathcal{W}(\phi)$, where $\mathcal{W}(\phi) \equiv \phi+W$ and $r W=\alpha \int \phi_{i}(a) d H(a, i)$. Similarly, the payoff to the investor from an agreement $\left(a^{\prime}, \phi\right)$ reached at time $\tau \geq t$ when his preference type is $i$ is $e^{-r(\tau-t)} \mathcal{U}_{i}\left(a^{\prime}, \phi\right)$, where $\mathcal{U}_{i}\left(a^{\prime}, \phi\right) \equiv V_{i}\left(a^{\prime}\right)-p\left(a^{\prime}-a\right)-\phi$, with

$$
V_{i}(a)=\frac{\bar{u}_{i}(a)+\kappa\left(p a+\Omega_{i}\right)}{r+\kappa}
$$

$\Omega_{i}=\frac{r+\kappa}{r+\delta+\kappa} \Delta_{i}+\frac{\delta}{r+\delta+\kappa} \sum_{j=1}^{I} \pi_{j} \Delta_{j}$ and $\Delta_{i}=\max _{a^{\prime} \geq 0}\left[\bar{u}_{i}\left(a^{\prime}\right)-r p a^{\prime}\right]$. If no agreement is reached, the (current value of) the payoff is $\mathcal{W}(0)$ to the dealer and $\mathcal{U}_{i}(a, 0)$ to the investor. There are gains from trade between an investor with preference type $i$ who holds $a$ and a dealer if

$$
\Gamma_{i}(a) \equiv \max _{a^{\prime} \geq 0}\left[V_{i}\left(a^{\prime}\right)-p a^{\prime}\right]-\left[V_{i}(a)-p a\right]>0,
$$

i.e., as long as $a \neq a_{i} \equiv \arg \max _{a^{\prime} \geq 0}\left[V_{i}\left(a^{\prime}\right)-p a^{\prime}\right]$. For the time being, we focus on the case where (55) holds; the analysis will be completed toward the end of the section.

A strategy for a player is a contingent plan that indicates which offer to make following every history after which it is the player's turn to make an offer, and which offers to accept or reject after every history where it is the player's turn to respond. The equilibrium concept we adopt is subgame perfect equilibrium, and we restrict attention to stationary strategies. ${ }^{25}$ The

\footnotetext{
${ }^{25}$ In our context, a strategy profile is stationary if the actions prescribed at any history depend only on the
} 
fact that the investor's preference type follows a stochastic process implies that the gains from trade also follow a stochastic process. In this setting, Merlo and Wilson (1995) have shown that agreement may turn out to be delayed in the subgame perfect equilibrium. We carry out the analysis under the conjecture that an agreement will not be delayed, and later verify that this is indeed the case for the particular parametrization of the game that we deem relevant for our application.

Let $\left(a_{i}^{1}, \phi_{i}^{1}\right)$ denote the proposal that the dealer makes to an investor of type $i$ who is holding $a$, and $\left(a_{i}^{2}, \phi_{i}^{2}\right)$ denote the offer that the latter makes to the former. ${ }^{26}$ Assuming the offers $\left\{\left(a_{i}^{2}, \phi_{i}^{2}\right)\right\}_{i=1}^{I}$ are accepted by dealers, the set of offers that an investor of type $i$ who is holding asset position $a$ accepts is

$$
\begin{gathered}
\mathcal{A}_{i}^{2}(a)=\left\{\left(a^{\prime}, \phi\right): \mathcal{U}_{i}\left(a^{\prime}, \phi\right) \geq \frac{1-\beta \Delta}{1+r \Delta}\left[\delta \Delta \sum_{j=1}^{I} \sum_{k=1}^{2} \pi_{j} \eta_{k} \mathcal{U}_{j}\left(a_{j}^{k}, \phi_{j}^{k}\right)\right.\right. \\
\left.\left.+(1-\delta \Delta) \sum_{k=1}^{2} \eta_{k} \mathcal{U}_{i}\left(a_{i}^{k}, \phi_{i}^{k}\right)\right]+\frac{\beta \Delta}{1+r \Delta}\left[\delta \Delta \sum_{j=1}^{I} \pi_{j} \mathcal{U}_{j}(a, 0)+(1-\delta \Delta) \mathcal{U}_{i}(a, 0)\right]\right\} .
\end{gathered}
$$

On the left side of the inequality that defines the set $\mathcal{A}_{i}^{2}(a)$ is the utility to the investor with preference type $i$ and asset holdings $a$ from immediate agreement to an offer $\left(a^{\prime}, \phi\right)$. On the right side of the inequality is his discounted expected utility from rejecting such an offer: he may receive a preference shock (with probability approximately equal to $\delta \Delta$ ), negotiations may break down (with probability approximately equal to $\beta \Delta$ ), and in the event that negotiations do not break down, he is selected to make an offer with probability $\eta_{2}$ and to respond to the dealer's offer with probability $\eta_{1}$. Similarly, the acceptance set of a dealer is

$\mathcal{A}^{1}=\left\{\left(a^{\prime}, \phi\right): \mathcal{W}(\phi) \geq \frac{1-\beta \Delta}{1+r \Delta}\left[\delta \Delta \sum_{j=1}^{I} \sum_{k=1}^{2} \pi_{j} \eta_{k} \mathcal{W}\left(\phi_{j}^{k}\right)+(1-\delta \Delta) \sum_{k=1}^{2} \eta_{k} \mathcal{W}\left(\phi_{i}^{k}\right)\right]+\frac{\beta \Delta}{1+r \Delta} \mathcal{W}(0)\right\}$.

current offer and the investor's current asset holdings and preference type. A subgame perfect equilibrium in stationary strategies is often referred to as a stationary subgame perfect equilibrium. Since we find a unique stationary subgame perfect equilibrium outcome, the restriction to stationary strategies is innocuous for our purposes because the subgame perfect equilibrium outcome of the bargaining game we are considering is unique if and only if the stationary subgame perfect equilibrium outcome is unique. See Merlo and Wilson (1995).

${ }^{26}$ We could write $a_{i}^{k}=a_{i}^{k}(a)$ and $\phi_{i}^{k}=\phi_{i}^{k}(a)$ to emphasize the fact that the offers may depend not only on the investor's preference type but also on his asset holdings. However, as we show below, the equilibrium offers $a_{i}^{1}, a_{i}^{2}$, and $\phi_{i}^{2}$ are independent of $a$ for all $i$. The fee proposed by the dealer, $\phi_{i}^{1}$, will depend on the investor's asset holdings $a$, so when $\phi_{i}^{1}$ appears, we really mean $\phi_{i}^{1}(a)$. 
Under the conjecture that agreement is not delayed in equilibrium, when it is his turn to propose, an investor of type $i$ chooses the offer that maximizes his own payoff from the set of offers that are acceptable to the dealer, i.e.,

$$
\left(a_{i}^{2}, \phi_{i}^{2}\right)=\arg \max _{\left(a^{\prime}, \phi\right) \in \mathcal{A}^{1}} \mathcal{U}_{i}\left(a^{\prime}, \phi\right),
$$

where the maximization is subject to $a^{\prime} \geq 0$. It is easy to show that $a_{i}^{2}=a_{i}$ and $\phi_{i}^{2}$ satisfies

$$
\mathcal{W}\left(\phi_{i}^{2}\right)=\frac{1-\beta \Delta}{1+r \Delta}\left[\delta \Delta \sum_{j=1}^{I} \sum_{k=1}^{2} \pi_{j} \eta_{k} \mathcal{W}\left(\phi_{j}^{k}\right)+(1-\delta \Delta) \sum_{k=1}^{2} \eta_{k} \mathcal{W}\left(\phi_{i}^{k}\right)\right]+\frac{\beta \Delta}{1+r \Delta} \mathcal{W}(0)
$$

for $i=1, \ldots, I$, which implies

$$
\phi_{i}^{2}=\frac{(1-\beta \Delta) \eta_{1}}{1+r \Delta-(1-\beta \Delta)(1-\delta \Delta) \eta_{2}}\left[(1-\delta \Delta) \phi_{i}^{1}+\frac{(1+r \Delta) \delta \Delta}{1+r \Delta-(1-\beta \Delta) \eta_{2}} \sum_{j=1}^{I} \pi_{j} \phi_{j}^{1}\right]-\frac{r \Delta}{1+r \Delta-(1-\beta \Delta) \eta_{2}} W
$$

Similarly, the optimal acceptable offer made by a dealer to an investor of type $i$ who is holding $a$ solves

$$
\left(a_{i}^{1}, \phi_{i}^{1}\right)=\arg \max _{\left(a^{\prime}, \phi\right) \in \mathcal{A}_{i}^{2}(a)} \mathcal{W}(\phi),
$$

where the maximization is subject to $a^{\prime} \geq 0$. For all $i$, the solution has $a_{i}^{1}=a_{i}$ and $\phi_{i}^{1}$ satisfies

$$
\begin{aligned}
\mathcal{U}_{i}\left(a_{i}, \phi_{i}^{1}\right) & =\frac{1-\beta \Delta}{1+r \Delta}\left[\delta \Delta \sum_{j=1}^{I} \sum_{k=1}^{2} \pi_{j} \eta_{k} \mathcal{U}_{j}\left(a_{j}, \phi_{j}^{k}\right)+(1-\delta \Delta) \sum_{k=1}^{2} \eta_{k} \mathcal{U}_{i}\left(a_{i}, \phi_{i}^{k}\right)\right] \\
& +\frac{\beta \Delta}{1+r \Delta}\left[\delta \Delta \sum_{j=1}^{I} \pi_{j} \mathcal{U}_{j}(a, 0)+(1-\delta \Delta) \mathcal{U}_{i}(a, 0)\right]
\end{aligned}
$$

which implies

$$
\phi_{i}^{1}=\frac{1-\beta \Delta}{1+r \Delta}\left[\delta \Delta \sum_{k=1}^{2} \sum_{j=1}^{I} \eta_{k} \pi_{j} \phi_{j}^{k}+(1-\delta \Delta) \sum_{k=1}^{2} \eta_{k} \phi_{i}^{k}\right]+G_{i}(a)
$$

where

$$
G_{i}(a)=\frac{1+r \Delta-(1-\beta \Delta)(1-\delta \Delta)}{1+r \Delta} \Gamma_{i}(a)-\frac{(1-\beta \Delta) \delta \Delta}{1+r \Delta} \bar{\Gamma}(a)+\frac{(r+\delta) \Delta}{1+r \Delta} V_{i}(a)-\frac{\delta \Delta}{1+r \Delta} \bar{V}(a),
$$

with $\bar{\Gamma}(a)=\sum_{j=1}^{I} \pi_{j} \Gamma_{j}(a)$ and $\bar{V}(a)=\sum_{j=1}^{I} \pi_{j} V_{j}(a)$. Combine (56) and (57) to arrive at

$$
\begin{aligned}
\phi_{i}^{1} & =\frac{(1+r \Delta)(1-\beta \Delta) \eta_{1} \delta \Delta}{[1+r \Delta-(1-\beta \Delta)(1-\delta \Delta)][1+r \Delta-(1-\beta \Delta)]}\left[\sum_{j=1}^{I} \pi_{j} G_{j}(a)-\frac{r \Delta(1-\beta \Delta) \eta_{2}}{(1+r \Delta)\left[1+r \Delta-(1-\beta \Delta) \eta_{2}\right]} W\right] \\
& +\frac{1+r \Delta-(1-\beta \Delta)(1-\delta \Delta) \eta_{2}}{1+r \Delta-(1-\beta \Delta)(1-\delta \Delta)}\left[G_{i}(a)-\frac{r \Delta(1-\beta \Delta) \eta_{2}}{(1+r \Delta)\left[1+r \Delta-(1-\beta \Delta) \eta_{2}\right]} W\right]
\end{aligned}
$$


and

$$
\begin{aligned}
\phi_{i}^{2} & =\frac{(1+r \Delta)(1-\beta \Delta) \eta_{1} \delta \Delta}{[1+r \Delta-(1-\beta \Delta)(1-\delta \Delta)][1+r \Delta-(1-\beta \Delta)]}\left[\sum_{j=1}^{I} \pi_{j} G_{j}(a)-\frac{r \Delta(1-\beta \Delta) \eta_{2}}{(1+r \Delta)\left[1+r \Delta-(1-\beta \Delta) \eta_{2}\right]} W\right] \\
& +\frac{(1-\beta \Delta)(1-\delta \Delta) \eta_{1}}{1+r \Delta-(1-\beta \Delta)(1-\delta \Delta)}\left[G_{i}(a)-\frac{r \Delta(1-\beta \Delta) \eta_{2}}{(1+r \Delta)\left[1+r \Delta-(1-\beta \Delta) \eta_{2}\right]} W\right]-\frac{r \Delta}{1+r \Delta-(1-\beta \Delta) \eta_{2}} W
\end{aligned}
$$

Next, we follow Binmore (1987) and consider the case where the time between each offer and counteroffer vanishes. This means that, no matter what the expected duration of the match may be (i.e., for any $\beta$ ), the dealer and the investor may in principle engage in an infinite number of bargaining rounds before the negotiation terminates. As we let $\Delta \rightarrow 0$, from (58) and (59) we find $\lim _{\Delta \rightarrow 0} \phi_{i}^{1}=\lim _{\Delta \rightarrow 0} \phi_{i}^{2}=\varphi_{i}(a, \beta)$, where

$$
\varphi_{i}(a, \beta)=\eta\left\{\Gamma_{i}(a)+\frac{r}{r+\beta} V_{i}(a)+\frac{\beta \delta}{(r+\beta)(r+\beta+\delta)}\left[V_{i}(a)-\bar{V}(a)\right]\right\} .
$$

Our theory is intended as a model of a fast-moving market with fleeting contacts between dealers and investors. To capture this idea, we consider the limit case where the average duration of the meeting between the dealer and the investor vanishes. As $\beta \rightarrow \infty$, we find $\varphi_{i}(a, \beta) \rightarrow \eta \Gamma_{i}(a)$. Then, since (55) and (54) imply $\Gamma_{i}(a)=\frac{\bar{u}_{i}\left(a_{i}\right)-\bar{u}_{i}(a)-r p\left(a_{i}-a\right)}{r+\kappa}$, we conclude that $\lim _{\beta \rightarrow \infty} \varphi_{i}(a, \beta)=\phi_{i}(a)$, where $\phi_{i}(a)$ is as in $(20)$.

To conclude, we verify that the sufficient condition in Merlo and Wilson (1998) that ensures that agreement will not be delayed for any investor-dealer pair in the subgame perfect equilibrium is satisfied as $\beta \rightarrow \infty$. The relevant condition is that the stochastic process for the discounted gains from trade is a supermartingale, which in our application reduces to

$$
\Gamma_{i}(a) \geq \frac{1-\beta \Delta}{1+r \Delta}\left[\delta \Delta \sum_{j=1}^{I} \pi_{j} \Gamma_{j}(a)+(1-\delta \Delta) \Gamma_{i}(a)\right]+o(\Delta),
$$

where $o(\Delta)$ is a function with the property that $\lim _{\Delta \rightarrow 0} \frac{o(\Delta)}{\Delta}=0$. If we rearrange this condition and let $\Delta$ go to zero, it becomes

$$
\Gamma_{i}(a) \geq \frac{\delta}{r+\beta+\delta} \bar{\Gamma}(a) .
$$

Since $\Gamma_{i}(a) \geq 0$ for all $i$ and $a$, this condition is satisfied as $\beta \rightarrow \infty$.

\section{C.3 Alternating offers in a discrete-time formulation of the model}

Here we consider a discrete-time version of our model in which the terms of trade in a bilateral match between a dealer and an investor correspond to the outcome of an alternating offers 
bargaining game with exogenous risk of breakdown. The environment is similar to the one laid out in the body of the paper, except that time is discrete and indexed by $t=0,1, \ldots$. The sequence of events within a time period is as follows. First, each investor enjoys utility $u_{i}(a)$ associated with his current preference type, $i$, and asset position, $a$. Second, investors receive preference shocks, and new contacts between dealers and investors take place. An investor receives a preference shock with probability $\delta$ and contacts a dealer with probability $\alpha$. We assume that these events are mutually exclusive and that $\alpha+\delta<1$. Third, the dealer-investor pairs bargain over the terms of the trade. We assume that although the negotiation occurs within the period, it can take place over an infinite number of bargaining rounds indexed by $\tau \in \mathbb{N}$. Lastly, the interdealer market opens and dealers execute trades on behalf of investors. The interdealer market closes when the period ends, and at that point all trades between dealers and investors are settled (assets are delivered and fees are paid) and all dealer-investor matches are dissolved.

Let $V_{i}(a, t)$ denote the maximum expected discounted utility attainable by an investor who has preference type $i$ and is holding $a$ at the end of period $t$, after the interdealer market has closed. It satisfies

$$
\begin{aligned}
(1+r) V_{i}(a, t-1) & =u_{i}(a)+\alpha\left\{V_{i}\left[a_{i}(t), t\right]-p(t)\left[a_{i}(t)-a\right]-\phi_{i}(a, t)\right\} \\
& +\delta \sum_{j=1}^{I} \pi_{j} V_{j}(a, t)+(1-\alpha-\delta) V_{i}(a, t)
\end{aligned}
$$

where $(1+r)^{-1}$ denotes the discount factor. Similarly, let $W(t)$ denote the maximum expected discounted utility attainable by a dealer at the end of period $t$, after the interdealer market has closed. It satisfies

$$
(1+r) W(t-1)=\alpha \int \phi_{i}(a, t) d H_{t}(a, i)+(1-\alpha) W(t) .
$$

We now turn to the determination of the terms of trade. We adopt the strategic model with exogenous risk of breakdown proposed by Binmore, Rubinstein, and Wolinsky (1986). Consider a meeting in period $t$ between a dealer and an investor of type $i$ who is holding $a$. The terms of trade are determined through an alternating offers bargaining game. The game takes place within the period, but it is composed of a large number of rounds. The dealer is the first proposer: he makes an offer that is accepted or rejected by the respondent. ${ }^{27}$ An offer is a pair

\footnotetext{
${ }^{27}$ Below we will eliminate the dealer's first-mover advantage by considering the limit of the game where the probabilities of breakdown between two consecutive rounds approach zero.
} 
$\left(a^{\prime}, \phi\right)$ that specifies the new asset position of the investor and the intermediation fee paid to the dealer. If rejected, the game moves to the second round where the investor can make a counteroffer. Between two consecutive rounds of negotiation the communication between the investor and the dealer can break down. The probability of breakdown following an offer by a dealer is $\eta_{1} \Delta$, while the probability of breakdown following an offer made by an investor is $\eta_{2} \Delta$. (The risk of breakdown acts as discounting between bargaining rounds and implies that agents strictly prefer not to delay the agreement.) The payoff to an investor with preference type $i$ if an agreement $\left(a^{\prime}, \phi\right)$ is reached at time $t$ is $\mathcal{U}_{i}\left(a^{\prime}, \phi, t\right) \equiv V_{i}\left(a^{\prime}, t\right)-p(t)\left(a^{\prime}-a\right)-\phi$, and the payoff to the dealer is $\mathcal{W}(\phi, t) \equiv \phi+W(t)$. If no agreement is reached, the payoff of the investor is $\mathcal{U}_{i}(a, 0, t)$, while the payoff of the dealer is $\mathcal{W}(0, t)$. There are gains from trade between an investor with preference type $i$ who holds $a$ and a dealer as long as

$$
\Gamma_{i}(a, t) \equiv \max _{a^{\prime} \geq 0}\left[V_{i}\left(a^{\prime}, t\right)-p(t) a^{\prime}\right]-\left[V_{i}(a, t)-p(t) a\right]>0,
$$

i.e., as long as $a \neq a_{i}(t) \equiv \arg \max _{a^{\prime} \geq 0}\left[V_{i}\left(a^{\prime}, t\right)-p(t) a^{\prime}\right]$. In what follows we focus on the case where $a \neq a_{i}(t)$ and assume that if $a=a_{i}(t)$, the dealer proposes the no-trade offer $(a, 0)$, which is accepted by the investor.

A bargaining strategy is a contingent plan that indicates which offer to make following every history after which it is the player's turn to make an offer, and which offers to accept or reject after every history where it is the player's turn to respond to an offer. The equilibrium concept we adopt is subgame perfect equilibrium, and we restrict attention to stationary strategies. ${ }^{28}$ Whenever it is his turn to make an offer, the dealer proposes $\left(a^{1}, \phi^{1}\right)$ while the investor proposes $\left(a^{2}, \phi^{2}\right)$. Assuming the offer $\left(a^{2}, \phi^{2}\right)$ is accepted by dealers, the acceptance set at time $t$ of an investor with preference type $i$ and asset holdings $a$ is

$$
\mathcal{A}_{i}^{2}(a, t)=\left\{\left(a^{\prime}, \phi\right): \mathcal{U}_{i}\left(a^{\prime}, \phi, t\right) \geq\left(1-\eta_{1} \Delta\right) \mathcal{U}_{i}\left(a^{2}, \phi^{2}, t\right)+\eta_{1} \Delta \mathcal{U}_{i}(a, 0, t)\right\} .
$$

A buyer accepts all offers that generate a payoff greater than or equal to his expected payoff if he rejects the offer and takes the chance to make a counteroffer. In the latter case, the investor makes the counteroffer $\left(a^{2}, \phi^{2}\right)$ with probability $1-\eta_{1} \Delta$, and with probability $\eta_{1} \Delta$ the negotiation breaks down. Similarly, the acceptance set of a dealer is

$$
\mathcal{A}^{1}(t)=\left\{\left(a^{\prime}, \phi\right): \mathcal{W}(\phi, t) \geq\left(1-\eta_{2} \Delta\right) \mathcal{W}\left(\phi^{1}, t\right)+\eta_{2} \Delta \mathcal{W}(0, t)\right\}
$$

\footnotetext{
${ }^{28}$ This restriction is innocuous, since the subgame perfect equilibrium of the bargaining game we are considering is unique. See Proposition 3 in Binmore, Rubinstein, and Wolinsky (1986) or Proposition 122.1 in Osborne and Rubinstein (1994).
} 
When it is his turn to make a proposal, the investor chooses the offer that maximizes his payoff among the set of offers that lie in the dealer's acceptance set, i.e., the investor's offer is

$$
\left\langle a_{i}^{2}(t), \phi_{i}^{2}(a, t)\right\rangle=\arg \max _{\left(a^{\prime}, \phi\right) \in \mathcal{A}^{1}(t)} \mathcal{U}_{i}\left(a^{\prime}, \phi, t\right)
$$

where the maximization is subject to $a^{\prime} \geq 0$. It is easy to show that $a_{i}^{2}(t)=a_{i}(t)$ and

$$
\phi_{i}^{2}(a, t)=\left(1-\eta_{2} \Delta\right) \phi_{i}^{1}(a, t)
$$

Similarly, the dealer's optimal offer when it is his turn to propose is

$$
\left\langle a_{i}^{1}(t), \phi_{i}^{1}(a, t)\right\rangle=\arg \max _{\left(a^{\prime}, \phi\right) \in \mathcal{A}_{i}^{2}(a, t)} \mathcal{W}(\phi, t)
$$

where the maximization is subject to $a^{\prime} \geq 0$. Hence, $a_{i}^{1}(t)=a_{i}(t)$ and $\phi_{i}^{1}(a, t)$ satisfies $\mathcal{U}_{i}\left[a_{i}(t), \phi_{i}^{1}(a, t), t\right]=\left(1-\eta_{1} \Delta\right) \mathcal{U}_{i}\left[a_{i}(t), \phi^{2}, t\right]+\eta_{1} \Delta \mathcal{U}_{i}(a, 0, t)$ or, equivalently,

$$
\Gamma_{i}(a, t)-\phi_{i}^{1}(a, t)=\left(1-\eta_{1} \Delta\right)\left[\Gamma_{i}(a, t)-\phi_{i}^{2}(a, t)\right]
$$

Use (65) to substitute $\phi_{i}^{2}(a, t)$ into this last expression and rearrange to get

$$
\phi_{i}^{1}(a, t)=\frac{\eta_{1} \Delta}{1-\left(1-\eta_{1} \Delta\right)\left(1-\eta_{2} \Delta\right)} \Gamma_{i}(a, t) .
$$

One can check that the offers $\left\langle a_{i}^{k}(t), \phi_{i}^{k}(a, t)\right\rangle$ for $k=1,2$, satisfy both agents' participation constraints. $^{29}$

From (65) and (66), we see that $\lim _{\Delta \rightarrow 0} \phi_{i}^{2}(a, t)=\lim _{\Delta \rightarrow 0} \phi_{i}^{1}(a, t)=\phi_{i}(a, t)$, where $\phi_{i}(a, t)$ is as in (4), with $\frac{\eta_{1}}{\eta_{1}+\eta_{2}} \equiv \eta$. It is possible to work out the rest of the model in discrete time. In particular, one could let the length of a period be given by $\Delta^{\prime}$, assume that the probabilities $\alpha$ and $\delta$, the rate of time preference, $r$, and the utility, $u_{i}(a)$, are all proportional to $\Delta^{\prime}$, and then let $\Delta^{\prime}$ approach zero to obtain the same expressions we have in the body of the paper.

\footnotetext{
${ }^{29}$ For the dealer, this only requires $\phi_{i}^{k}(a, t) \geq 0$ for $k=1,2$ and all $i$. From (66),

$$
\Gamma_{i}(a, t)-\phi_{i}^{1}(a, t)=\frac{\eta_{2} \Delta\left(1-\eta_{1} \Delta\right)}{1-\left(1-\eta_{1} \Delta\right)\left(1-\eta_{2} \Delta\right)} \Gamma_{i}(a, t) \geq 0
$$

where the inequality is strict provided $a \neq a_{i}(t)$. So $\left\langle a_{i}(t), \phi_{i}^{1}(a, t)\right\rangle$ is preferred by the investor to no trade. It can be easily checked that the same is true of $\left\langle a_{i}(t), \phi_{i}^{2}(a, t)\right\rangle$.
} 


\section{C.4 A single bargaining round in a discrete-time formulation of the model}

In the context of the model analyzed in Section C.3, we could, instead of considering a bargaining game with a potentially infinite number of rounds, adopt a simpler two-stage bargaining game that delivers the same outcome. In the first stage the dealer makes an offer that the investor accepts or rejects. Following the investor's decision to accept or reject, the bargaining ends with probability $\gamma \Delta$. (As in Section C.3, the risk of breakdown acts as discounting between bargaining rounds and implies that agents strictly prefer not to delay the agreement.) With probability $1-\gamma \Delta$ one of two players can make a counteroffer without delay. Nature chooses either the investor (with probability $1-\eta$ ) or the dealer (with probability $\eta$ ) to make the counteroffer. The recipient of the counteroffer chooses whether to accept or reject it, and regardless of his action, the game ends and the two players part ways.

If the investor is the proposer in the second stage, he offers $\left\langle a_{i}^{2}(t), \phi_{i}^{2}(a, t)\right\rangle=\left\langle a_{i}(t), 0\right\rangle$, where $a_{i}(t) \equiv \arg \max _{a^{\prime} \geq 0}\left[V_{i}\left(a^{\prime}, t\right)-p(t) a^{\prime}\right]$. If the dealer is the proposer in the second stage, he offers $\left\langle a_{i}(t), \Gamma_{i}(a, t)\right\rangle$ where $\Gamma_{i}(a, t)$ is as in (62). Thus, in the first stage the dealer offers

$$
\begin{gathered}
\left\langle a_{i}^{1}(t), \phi_{i}^{1}(a, t)\right\rangle=\arg \max _{\left(a^{\prime}, \phi\right)} \phi \\
\text { s.t. } \quad V_{i}\left(a^{\prime}, t\right)-p(t)\left(a^{\prime}-a\right)-\phi-V_{i}(a, t) \geq(1-\gamma \Delta)(1-\eta) \Gamma_{i}(a, t)
\end{gathered}
$$

and $a^{\prime} \geq 0$. It is easy to see that $a_{i}^{1}(t)=a_{i}(t)$ and $\phi_{i}^{1}(a, t)=[1-(1-\gamma \Delta)(1-\eta)] \Gamma_{i}(a, t)$. Therefore, also in this game we find $\lim _{\Delta \rightarrow 0} \phi_{i}^{1}(a, t)=\phi_{i}(a, t)$, where $\phi_{i}(a, t)$ is as in (4). 


\section{Principle of optimality}

Consider an investor who effectively contacts the market with Poisson intensity $\kappa$, and who is subject to preference shocks with Poisson intensity $\delta$. Let $\left\{T_{n}\right\}_{n=1}^{\infty}$ denote the sequence of contact times, and $N_{t}$ the number of contacts over the time interval $[0, t)$. Similarly, let $\left\{T_{n}^{\prime}\right\}_{n=1}^{\infty}$ denote the sequence of times at which he receives preference shocks. We adopt the convention that $T_{0}=T_{0}^{\prime}=0$. Define the function $k: \mathbb{R}_{+} \rightarrow \mathbb{X}$, and interpret $k(t)$ as the investor's preference type at time $t$. The process for preference shocks implies $k(t)=k\left(T_{n}^{\prime}\right)$ for $t \in\left[T_{n}^{\prime}, T_{n+1}^{\prime}\right)$, for any integer $n \geq 0$. The realization $\omega=\left(N_{t}, k(t)\right)_{t \in[0, \infty)}$ summarizes an investor's individual history of shocks. Let $\Omega$ be the set of all such histories. Similarly, let $\omega^{t}=\left(N_{s}, k(s)\right)_{s \in[0, t]}$ denote a history of shocks up to time $t$, and $\Omega^{t}$ be the collection of all such histories. We work with the probability space $(\Omega, \mathcal{H}, \mathbb{P})$, where $\mathcal{H}$ is an appropriate $\sigma$-field of subsets of $\Omega$ (e.g., the $\sigma$-field generated by $\Omega^{t}$ for all finite $t$ ), and $\mathbb{P}$ is the probability measure on $\mathcal{H}$ induced by the independent Poisson processes for preference shocks and effective contacts with the market. Let $\mathcal{H}^{t} \subseteq \mathcal{H}$ be a partition of $\Omega$ such that $H_{\varpi}^{t} \in \mathcal{H}^{t}$ is a set of histories that coincide over $[0, t]$, i.e., $H_{\varpi}^{t}=\left\{\omega \in \Omega: \omega^{t}=\varpi\right.$ for some $\left.\varpi \in \Omega^{t}\right\}$. The $\sigma$-field generated by $\mathcal{H}^{t}$, denoted $\mathcal{F}^{t}$, captures the information available to the investor at time $t$, and the filtration $\left\{\mathcal{F}^{t}, t \in \mathbb{R}_{+}\right\}$represents how information is revealed over time.

An asset plan, $\mathbf{a}=\left(\boldsymbol{a}_{t}\right)_{t \in[0, \infty)}$, for the investor is a set of functions $\boldsymbol{a}_{t}: \Omega \rightarrow[0, \bar{a}]$ for all $t \geq 0$, such that $\boldsymbol{a}_{t}$ is $\mathcal{F}^{t}$-measurable. ${ }^{30}$ An asset plan $\left(\boldsymbol{a}_{t}\right)_{t \in[0, \infty)}$ is feasible if for every $\omega, \boldsymbol{a}_{0}(\omega)$ equals the given initial asset holding of the investor, and $\boldsymbol{a}_{t}(\omega)=\boldsymbol{a}_{T_{n}}(\omega)$ for all $t \in\left[T_{n}, T_{n+1}\right)$. Let $\mathbb{A}$ denote the set of all feasible asset plans. Let $\mathcal{U}_{k(t)}^{M}(\cdot, t)$ be the utility functional over the time interval $\left[t, T_{M}\right]$ of an investor with preference type $k(t)$ at time $t$. His utility over the period $\left[t, T_{M}\right]$ from following asset plan $\mathbf{a}=\left(\boldsymbol{a}_{s}\right)_{s \in[0, \infty)}$ is

$$
\begin{aligned}
\mathcal{U}_{k(t)}^{M}(\mathbf{a}, t) & =\mathbb{E}_{t}\left[\int_{t}^{T_{N_{t}+1}} e^{-r(s-t)} u_{k(s)}\left(\boldsymbol{a}_{t}(\omega)\right) d s+\sum_{n=1}^{M-1} \int_{T_{N_{t}+n}}^{T_{N_{t}+n+1}} e^{-r(s-t)} u_{k(s)}\left(\boldsymbol{a}_{T_{N_{t}+n}}(\omega)\right) d s\right. \\
& -e^{-r\left(T_{N_{t}+1}-t\right)} p\left(T_{N_{t}+1}\right)\left[\boldsymbol{a}_{T_{N_{t}+1}}(\omega)-\boldsymbol{a}_{t}(\omega)\right] \\
& \left.-\sum_{n=1}^{M-1} e^{-r\left(T_{N_{t}+n+1}-t\right)} p\left(T_{N_{t}+n+1}\right)\left[\boldsymbol{a}_{T_{N_{t}+n+1}}(\omega)-\boldsymbol{a}_{T_{N_{t}+n}}(\omega)\right]\right]
\end{aligned}
$$

\footnotetext{
${ }^{30}$ The upper bound $\bar{a}$ is imposed for technical reasons (to ensure that the investor's utility is bounded above), and is chosen to be sufficiently large so that it does not affect the investor's decision.
} 
where $\mathbb{E}_{t}$ is shorthand for the conditional expectation $\mathbb{E}\left[\cdot \mid \mathcal{F}^{t}\right] .31$ The first $M$ terms on the right side of (67) represent the expected discounted sum of utility flows from holding the asset position prescribed by the asset plan a over the time interval $\left[t, T_{N_{t}+M}\right)$. The first term, for instance, is the expected utility from holding the asset position $\boldsymbol{a}_{t}(\omega)$ from the initial time $t$ until the next time the investor gains effective access to the market, $T_{N_{t}+1}$. Similarly, each term in the summation represents the utility from holding the asset over the period $\left[T_{N_{t}+n}, T_{N_{t}+n+1}\right)$, i.e., between the effective contact number $N_{t}+n$ and the next one. The second $M$ terms represent the expected net utility cost to the investor from readjusting his asset holdings at the times he contacts the market. The term on the second line of (67), for instance, is the (expected, discounted to time $t$ ) disutility the investor incurs to buy $\boldsymbol{a}_{T_{N_{t}+1}}(\omega)$ on his $\left(N_{t}+1\right)^{\text {th }}$ effective contact with the market, net of the utility he gets from selling the assets he is holding at this time, $\boldsymbol{a}_{t}(\omega)$. In what follows, we will leave the dependence of the function $\boldsymbol{a}_{t}$ on $\omega$ implicit to simplify the notation. By the Law of Iterated Expectations, the utility functional in (67) can be rewritten as

$$
\begin{aligned}
\mathcal{U}_{k(t)}^{M}(\mathbf{a}, t) & =\frac{\bar{u}_{k(t)}\left(\boldsymbol{a}_{t}\right)}{r+\kappa}+\left[p(t)-\frac{q(t)}{r+\kappa}\right] \boldsymbol{a}_{t}-\mathbb{E}_{t}\left[e^{-r\left(T_{N_{t}+M}-t\right)} p\left(T_{N_{t}+M}\right) \boldsymbol{a}_{T_{N_{t}+M}}\right] \\
& +\frac{1}{r+\kappa} \mathbb{E}_{t}\left\{\sum_{n=1}^{M-1} e^{-r\left(T_{N_{t}+n}-t\right)}\left[\bar{u}_{k\left(T_{N_{t}+n}\right)}\left(\boldsymbol{a}_{T_{N_{t}+n}}\right)-q\left(T_{N_{t}+n}\right) \boldsymbol{a}_{T_{N_{t}+n}}\right]\right\}
\end{aligned}
$$

where

$$
\begin{aligned}
\bar{u}_{k\left(T_{N_{t}+n}\right)}\left(\boldsymbol{a}_{T_{N_{t}+n}}\right) & \equiv(r+\kappa) \mathbb{E}_{T_{N_{t}+n}} \int_{T_{N_{t}+n}}^{T_{N_{t}+n+1}} e^{-r\left(s-T_{N_{t}+n}\right)} u_{k(s)}\left(\boldsymbol{a}_{T_{N_{t}+n}}\right) d s \\
q\left(T_{N_{t}+n}\right) & \equiv(r+\kappa)\left[p\left(T_{N_{t}+n}\right)-\mathbb{E}_{T_{N_{t}+n}} e^{-r\left(T_{N_{t}+n+1}-T_{N_{t}+n}\right)} p\left(T_{N_{t}+n+1}\right)\right]
\end{aligned}
$$

Notice that the function $\bar{u}_{i}(a)$ is as in (7), and since

$$
\mathbb{E}_{T_{N_{t}+n}} e^{-r\left(T_{N_{t}+n+1}-T_{N_{t}+n}\right)} p\left(T_{N_{t}+n+1}\right)=\kappa \int_{0}^{\infty} e^{-(r+\kappa) s} p\left(T_{N_{t}+n}+s\right) d s,
$$

the function $q(t)$ is the one defined in (8). For any finite $M$, and any $t$, the utility functional $\mathcal{U}_{k(t)}^{M}(\mathbf{a}, t)$ is well-defined for any feasible asset plan $\mathbf{a} \cdot{ }^{32}$

\footnotetext{
${ }^{31}$ Notice that the stochastic process $\left\{T_{n}\right\}_{n=1}^{\infty}$ can be thought of as being a function of the process $\omega$ (since $\left(N_{t}\right)_{t \in[0, \infty)}$ is a right-continuous step function with jumps at $\left.\left\{T_{n}\right\}_{n=1}^{\infty}\right)$, so for any $\mathcal{F}^{t}$-measurable function $f: \Omega \rightarrow \mathbb{R} \cup\{ \pm \infty\}$, the expectation $\mathbb{E}\left[f(\omega) \mid \mathcal{F}^{t}\right]$ is also integrating over $\left\{T_{n}\right\}_{n=1}^{\infty}$.

${ }^{32}$ From (7), it is clear that the first term on the right side of (68) is a well-behaved function of $\boldsymbol{a}_{t}$, which is itself a bounded and $\mathcal{F}^{t}$-measurable function. Since throughout the paper we have specialized the analysis to
} 
Next, for any given nonnegative measurable price function $p(t)$, we define the infinitehorizon utility for the investor from following a feasible asset plan a, by

$$
\mathcal{U}_{k(t)}(\mathbf{a}, t)=\limsup _{M \rightarrow \infty} \mathcal{U}_{k(t)}^{M}(\mathbf{a}, t)
$$

For any feasible asset plan, the sequence

$$
\left\{\sum_{n=1}^{M-1} e^{-r\left(T_{N_{t}+n}-t\right)} \bar{u}_{k\left(T_{N_{t}+n}\right)}\left(\boldsymbol{a}_{T_{N_{t}+n}}\right)\right\}_{M=1}^{\infty}
$$

has a limit. This limit may be a finite number, or $-\infty .{ }^{33}$ The sequence

$$
\left\{\sum_{n=1}^{M-1} e^{-r\left(T_{N_{t}+n}-t\right)} q\left(T_{N_{t}+n}\right) \boldsymbol{a}_{T_{N_{t}+n}}\right\}_{M=1}^{\infty}
$$

is nondecreasing, so it has a limit, which may be $+\infty$. Let

$$
f_{M}(\omega) \equiv \sum_{n=1}^{M-1} e^{-r\left(T_{N_{t}+n}-t\right)}\left[\bar{u}_{k\left(T_{N_{t}+n}\right)}\left(\boldsymbol{a}_{T_{N_{t}+n}}\right)-q\left(T_{N_{t}+n}\right) \boldsymbol{a}_{T_{N_{t}+n}}\right],
$$

then we have shown that $\lim _{M \rightarrow \infty} f_{M}$ exists (it may be finite or $-\infty$ ). If we rescale $u_{i}$ for each $i$ so that $u_{i}(\bar{a}) \leq 0$ for all $i$, we see that the sequence $\left\{-f_{M}\right\}_{M=1}^{\infty}$ is a monotone increasing sequence of measurable functions that converge pointwise to $-\lim _{M \rightarrow \infty} f_{M}$, so by the Monotone Convergence Theorem (e.g., Theorem 7.8 in Stokey and Lucas, 1989), we have $\lim _{M \rightarrow \infty} \mathbb{E}_{t}\left[f_{M}\right]=\mathbb{E}_{t}\left[\lim _{M \rightarrow \infty} f_{M}\right]$. All this implies that, given a price path $p(t)$, an investor's expected lifetime utility from following a feasible asset plan $\mathbf{a}=\left(\boldsymbol{a}_{s}\right)_{s \in[0, \infty)}$ is

$$
\begin{aligned}
\mathcal{U}_{k(t)}(\mathbf{a}, t) & =\frac{\bar{u}_{k(t)}\left(\boldsymbol{a}_{t}\right)}{r+\kappa}+\left[p(t)-\frac{q(t)}{r+\kappa}\right] \boldsymbol{a}_{t}-\limsup _{M \rightarrow \infty} \mathbb{E}_{t}\left[e^{-r\left(T_{N_{t}+M}-t\right)} p\left(T_{N_{t}+M}\right) \boldsymbol{a}_{T_{N_{t}+M}}\right] \\
& +\frac{1}{r+\kappa} \mathbb{E}_{t}\left\{\sum_{n=1}^{\infty} e^{-r\left(T_{N_{t}+n}-t\right)}\left[\bar{u}_{k\left(T_{N_{t}+n}\right)}\left(\boldsymbol{a}_{T_{N_{t}+n}}\right)-q\left(T_{N_{t}+n}\right) \boldsymbol{a}_{T_{N_{t}+n}}\right]\right\},
\end{aligned}
$$

price paths with the property that $p(t)$ is measurable, $q(t)$ is well-defined for any $t$, and the second term on the right side of $(68)$ is well-defined. Since $e^{-r t} p(t) \boldsymbol{a}_{t}$ is a nonnegative measurable function, the integral in the third term is well-defined (although it need not be finite). As for the last term, notice that $\bar{u}_{k\left(T_{N_{t}+n}\right)}(a)=$ $\sum_{i=1}^{I} \bar{u}_{i}(a) \mathbb{I}_{\left\{k\left(T_{N_{t}+M}\right)=i\right\}}$, where $\bar{u}_{i}(a)$ is a continuous function for each $i$, so the integral of $e^{-r t} \bar{u}_{k(t)}\left(\boldsymbol{a}_{t}\right)$ is well-defined. Finally, the integral of $q(t) \boldsymbol{a}_{t}$ is well-defined since $p(t)$ and $\boldsymbol{a}_{t}$ are nonnegative and measurable.

${ }^{33}$ This limit is finite if $u_{i}$ is bounded below for all $i$, since in that case we can rescale each utility function so that $u_{i}(0) \geq 0$ for all $i$, and the sequence of partial sums is nondecreasing and bounded above (because $a_{t} \leq \bar{a}$ for all $t$, and $u_{i}$ is continuous for each $i$ ). Conversely, if some $u_{i}$ is unbounded below, we can rescale $u_{i}$ and every other $u_{j}$ so that $u_{k}(\bar{a}) \leq 0$ for all $k$. Then since the sequence of partial sums is nonincreasing, it has a limit, which could be $-\infty$. 
which is well-defined for any feasible path. ${ }^{34}$ The investor's problem at $t$ is

$$
\max _{\mathbf{a} \in \mathbb{A}} \mathcal{U}_{k(t)}(\mathbf{a}, t) \text {, s.t. } a_{t}=a \geq 0, \text { and } k(t) \in \mathbb{X} \text {, given. }
$$

The investor's maximum attainable utility is then

$$
V_{k(t)}^{*}(a, t)=\max _{\mathbf{a} \in \mathbb{A}} \mathcal{U}_{k(t)}(\mathbf{a}, t) .
$$

Proposition 12 A feasible plan $\mathbf{a}^{*}=\left(\boldsymbol{a}_{s}^{*}(\omega)\right)_{s \in[t, \infty), \omega \in \Omega}$ is optimal from a given initial date $t \geq 0$ if and only if it satisfies

$$
\boldsymbol{a}_{T_{n}}^{*}(\omega)=\arg \max _{a \in[0, \bar{a}]}\left[\bar{u}_{k\left(T_{n}\right)}(a)-q\left(T_{n}\right) a\right], \quad \forall \omega \in \Omega, \forall\left\{T_{n}\right\}_{n=T_{N_{t}+1}}^{\infty}
$$

and

$$
\lim _{n \rightarrow \infty} \mathbb{E}_{i}\left\{e^{-r\left(T_{N_{t}+n}-t\right)} p\left(T_{N_{t}+n}\right) \boldsymbol{a}_{T_{N_{t}+n}}^{*}\right\}=0 .
$$

Moreover, if there exists a number $B>\max _{j} \bar{u}_{j}^{\prime}(\infty)$ such that $q(s) \geq B$ for all $s$, then an optimal plan exists and is unique.

Proof. The proof proceeds in three steps. Step $(i)$ : We first show that (72) and (73) are sufficient for an optimum. Let $\mathbf{a}^{*}$ be the asset plan that satisfies (72) and (73), and $\mathbf{a}$ be any other feasible plan. For any $t$, let $\Delta \equiv \mathcal{U}_{k(t)}\left(\mathbf{a}^{*}, t\right)-\mathcal{U}_{k(t)}(\mathbf{a}, t)$, then

$$
\begin{aligned}
\Delta & \geq \frac{1}{r+\kappa} \mathbb{E}_{t}\left\{\sum_{n=1}^{\infty} e^{-r\left(T_{N_{t}+n}-t\right)}\left[\bar{u}_{k\left(T_{N_{t}+n}\right)}\left(\boldsymbol{a}_{T_{N_{t}+n}}^{*}\right)-q\left(T_{N_{t}+n}\right) \boldsymbol{a}_{T_{N_{t}+n}}^{*}\right]\right\} \\
& -\frac{1}{r+\kappa} \mathbb{E}_{t}\left\{\sum_{n=1}^{\infty} e^{-r\left(T_{N_{t}+n}-t\right)}\left[\bar{u}_{k\left(T_{N_{t}+n}\right)}\left(\boldsymbol{a}_{T_{N_{t}+n}}\right)-q\left(T_{N_{t}+n}\right) \boldsymbol{a}_{T_{N_{t}+n}}\right]\right\} \\
& -\limsup _{M \rightarrow \infty} \mathbb{E}_{t}\left[e^{-r\left(T_{N_{t}+M}-t\right)} p\left(T_{N_{t}+M}\right) \boldsymbol{a}_{T_{N_{t}+M}}^{*}\right] .
\end{aligned}
$$

From (72) and (73), it follows that $\Delta \geq 0$. Step (ii): Next, we show that an optimal plan must satisfy (72) and (73). The first step is to notice that the objective function on the right side of

\footnotetext{
${ }^{34} \mathrm{We}$ have chosen to define the lifetime utility as $\limsup _{M \rightarrow \infty} \mathcal{U}^{M}(\mathbf{a}, t)$ rather than $\lim _{M \rightarrow \infty} \mathcal{U}^{M}(\mathbf{a}, t)$, because $\lim _{M \rightarrow \infty} \mathbb{E}_{t}\left[e^{-r\left(T_{N_{t}+M}-t\right)} p\left(T_{N_{t}+M}\right) a_{T_{N_{t}+M}}\right]$ need not exist for every feasible asset plan. The definition we have adopted guarantees that the payoff from every feasible asset plan can be evaluated using the investor's utility function. As we show below, the optimal asset plan, $\mathbf{a}^{*}$, has the property that $\lim _{M \rightarrow \infty} \mathbb{E}_{t}\left[e^{-r\left(T_{N_{t}+M}-t\right)} p\left(T_{N_{t}+M}\right) a_{T_{N_{t}+M}}^{*}\right]=0$, which means that, equivalently, we could define the utility function as $\lim _{M \rightarrow \infty} \mathcal{U}^{M}(\mathbf{a}, t)$, and simply restrict the investor's choices to the set of feasible paths for which $\lim _{M \rightarrow \infty} \mathbb{E}_{t}\left[e^{-r\left(T_{N_{t}+M}-t\right)} p\left(T_{N_{t}+M}\right) a_{T_{N_{t}+M}}\right]$ exists.
} 
(72) is strictly concave and differentiable, so $u_{i}^{\prime}\left[\boldsymbol{a}_{s}^{*}(\omega)\right]-q(s) \leq 0$ ("=" if $\left.\boldsymbol{a}_{i}^{*}(s)>0\right)$ is necessary and sufficient for an optimum. Since $q(s)>\bar{u}_{i}^{\prime}(\infty)$ for all $i$, we can choose $\bar{a}$ large enough so that $q(s)>\bar{u}_{i}^{\prime}(\bar{a})$ for all $i$, and therefore $(72)$ is the unique solution to the investor's problem at time $s$, for history $\omega$, when his preference type is $k(s)$. Suppose that the asset plan ã is optimal, with $\tilde{\boldsymbol{a}}_{s}(\omega) \neq \boldsymbol{a}_{s}^{*}(\omega)$ for some history $\omega$ at some date $s>t$. Since both $\tilde{\mathbf{a}}$ and $\mathbf{a}^{*}$ are feasible, $\tilde{\boldsymbol{a}}_{T_{N_{s}}}(\omega) \neq \boldsymbol{a}_{T_{N_{s}}}^{*}(\omega)$. Then the investor could maintain his asset plan ã unchanged except at date $T_{N_{s}}$ for history $\omega$, where he could choose $\boldsymbol{a}_{T_{N_{s}}}^{*}(\omega)$. By (72), this deviation is feasible. Since the maximization in (72) has a unique solution, the proposed deviation strictly increases the investor's expected utility, so ã could not have been optimal - a contradiction. Next, we show that any optimal policy must satisfy (73). Let $\mathbf{a}^{*}$ be an optimal plan, and consider the feasible plan $(1-\varepsilon) \mathbf{a}^{*}$, for some small $\varepsilon>0$. Let $\Delta_{\varepsilon} \equiv \mathcal{U}_{k(t)}\left(\mathbf{a}^{*}, t\right)-\mathcal{U}_{k(t)}\left[(1-\varepsilon) \mathbf{a}^{*}, t\right]$; then,

$$
\begin{aligned}
\Delta_{\varepsilon} & =\mathbb{E}_{t}\left\{\sum_{n=1}^{\infty} \frac{e^{-r\left(T_{N_{t}+n}-t\right)}}{r+\kappa}\left[\bar{u}_{k\left(T_{N_{t}+n}\right)}\left(\boldsymbol{a}_{T_{N_{t}+n}}^{*}\right)-\bar{u}_{k\left(T_{N_{t}+n}\right)}\left[(1-\varepsilon) \boldsymbol{a}_{T_{N_{t}+n}}^{*}\right]-\varepsilon q\left(T_{N_{t}+n}\right) \boldsymbol{a}_{T_{N_{t}+n}}^{*}\right]\right\} \\
& -\varepsilon \limsup _{M \rightarrow \infty}\left[\mathbb{E}_{t}\left[e^{-r\left(T_{N_{t}+M}-t\right)} p\left(T_{N_{t}+M}\right) \boldsymbol{a}_{T_{N_{t}+M}}^{*}\right] .\right.
\end{aligned}
$$

Divide the previous expression by $\varepsilon$, and take the limit as $\varepsilon \rightarrow 0$ (applying L'Hôpital's Rule) to arrive at

$$
\begin{aligned}
\lim _{\varepsilon \rightarrow 0} \frac{\Delta_{\varepsilon}}{\varepsilon} & =\frac{1}{r+\kappa} \mathbb{E}_{t}\left\{\sum_{n=1}^{\infty} e^{-r\left(T_{N_{t}+n}-t\right)}\left[\bar{u}_{k\left(T_{N_{t}+n}\right)}^{\prime}\left(\boldsymbol{a}_{T_{N_{t}+n}}^{*}\right)-q\left(T_{N_{t}+n}\right)\right] \boldsymbol{a}_{T_{N_{t}+n}}^{*}\right\} \\
& -\limsup _{M \rightarrow \infty} \mathbb{E}_{t}\left[e^{-r\left(T_{N_{t}+M}-t\right)} p\left(T_{N_{t}+M}\right) \boldsymbol{a}_{T_{N_{t}+M}}^{*}\right] .
\end{aligned}
$$

Since the asset plan $\mathbf{a}^{*}$ is optimal, the first-order condition for the investor's problem (72), i.e., $\left[\bar{u}_{k\left(T_{n}\right)}^{\prime}\left(\boldsymbol{a}_{T_{n}}^{*}\right)-q\left(T_{n}\right)\right] \boldsymbol{a}_{T_{n}}^{*}=0$ for all $\left\{T_{n}\right\}_{n=T_{N_{t}+1}}^{\infty}$, implies

$$
\lim _{\varepsilon \rightarrow 0} \frac{\Delta_{\varepsilon}}{\varepsilon}=-\limsup _{M \rightarrow \infty} \mathbb{E}_{t}\left[e^{-r\left(T_{N_{t}+M}-t\right)} p\left(T_{N_{t}+M}\right) \boldsymbol{a}_{T_{N_{t}+M}}^{*}\right],
$$

and the optimality of $\mathbf{a}^{*}$ requires

$$
0 \leq-\limsup _{M \rightarrow \infty}\left[\mathbb{E}_{t}^{-r\left(T_{N_{t}+M}-t\right)} p\left(T_{N_{t}+M}\right) \boldsymbol{a}_{T_{N_{t}+M}}^{*}\right]
$$

Then, since $e^{-r T} p(T) \boldsymbol{a}_{T}^{*} \geq 0$ for all $T$, we have

$0 \leq \liminf _{M \rightarrow \infty} \mathbb{E}_{t}\left[e^{-r\left(T_{N_{t}+M}-t\right)} p\left(T_{N_{t}+M}\right) \boldsymbol{a}_{T_{N_{t}+M}}^{*}\right] \leq \limsup _{M \rightarrow \infty} \mathbb{E}_{t}\left[e^{-r\left(T_{N_{t}+M}-t\right)} p\left(T_{N_{t}+M}\right) \boldsymbol{a}_{T_{N_{t}+M}}^{*}\right] \leq 0$, 
so the optimality of $\mathbf{a}^{*}$ requires

$$
\lim _{M \rightarrow \infty} \mathbb{E}_{t}\left[e^{-r\left(T_{N_{t}+M}-t\right)} p\left(T_{N_{t}+M}\right) \boldsymbol{a}_{T_{N_{t}+M}}^{*}\right]=0
$$

Step (iii): Finally, since the necessary conditions (72) and (73) determine a unique $\mathbf{a}^{*}=$ $\left(\boldsymbol{a}_{t}^{*}(\omega)\right)_{t \in[0, \infty), \omega \in \Omega}$, the optimal plan exists and is unique.

The formulation we have laid out in this appendix is quite general, in that it allows the investor to choose among feasible asset plans $\mathbf{a}=\left(\boldsymbol{a}_{t}(\omega)\right)_{t \in[0, \infty), \omega \in \Omega}$, where $\boldsymbol{a}_{t}$ can be any $\mathcal{F}^{t}$-measurable function of the whole history of shocks, $\omega$, as well as time, $t$. From (72), however, notice that the optimal asset plan $\mathbf{a}^{*}=\left(\boldsymbol{a}_{t}^{*}(\omega)\right)_{t \in[0, \infty), \omega \in \Omega}$ is not history-dependent: when the investor gains effective access to the market at time $T_{n}$, his optimal decision depends only on $T_{n}$ and his preference type at that time, $k\left(T_{n}\right)$. For this reason, we can simplify the notation as we did in the body of the paper, by letting $a_{k\left(T_{n}\right)}\left(T_{n}\right) \equiv \boldsymbol{a}_{T_{n}}^{*}(\omega)$. With this notation, we can denote the optimal plan $\mathbf{a}^{*}$ simply by a sequence of functions $\left\{\left(a_{i}(t), t \in[0, \infty)\right)\right\}_{i=1}^{I}$, with $a_{i}(t)=a_{i}\left(T_{n}\right)$ for all $t \in\left[T_{n}, T_{n+1}\right)$ and every $i$. Also as in the body of the paper, we can use $\mathbb{E}_{k(t)}$ to denote $\mathbb{E}_{t}$, which stresses the fact that $k(t)$ summarizes all the relevant information available to the investor at time $t$ in order to form the conditional expectation over $\omega$. With this notation, consider an investor at time $t$, with asset holdings $a_{t}=a \geq 0$, and preference type $k(t)=i \in \mathbb{X}$, both given. His maximum attainable utility is $V_{i}^{*}(a, t)=\mathcal{U}_{i}\left(\mathbf{a}^{*}, t\right)$, i.e.,

$$
V_{i}^{*}(a, t)=\frac{\bar{u}_{i}(a)}{r+\kappa}+\left[p(t)-\frac{q(t)}{r+\kappa}\right] a+K_{i}(t),
$$

where

$$
K_{i}(t)=\mathbb{E}_{i}\left\{\sum_{n=1}^{\infty} e^{-r\left(T_{N_{t}+n}-t\right)}\left[\frac{\bar{u}_{k\left(T_{N_{t}+n}\right)}\left[a_{k\left(T_{N_{t}+n}\right)}\left(T_{N_{t}+n}\right)\right]}{r+\kappa}-\frac{q\left(T_{N_{t}+n}\right)}{r+\kappa} a_{k\left(T_{N_{t}+n}\right)}\left(T_{N_{t}+n}\right)\right]\right\} .
$$

From Proposition 12 we know that if there exists a number $B>\max _{j} \bar{u}_{j}^{\prime}(\infty)$ such that $q(s) \geq B$ for all $s$, then an optimal plan $\left\{\left(a_{i}(t), t \in[0, \infty)\right)\right\}_{i=1}^{I}$ exists and is unique, so $K_{i}(t)$ is welldefined. If, in addition, there exists a real number $\bar{B}$ such that $q(t) \leq \bar{B}$ for all $t$, then $K_{i}(t) \in \mathbb{R}$ for all $t$ and every $i$.

Instead of considering (71), in the body of the paper we described the investor's problem using a recursive functional equation, i.e., (1) with asset holdings and fees given by (2), which we showed to be equivalent to (27). Lemma 8 formalizes the relationship between both formulations of the investor's problem, (27) and (71). Before we prove this result, it is convenient to establish a preliminary result. 
Lemma 7 For any $t \geq 0$,

$$
\begin{aligned}
K_{k(t)}(t) & =\frac{1}{r+\kappa} \mathbb{E}_{k(t)}\left\{e^{-r\left(T_{N_{t}+1}-t\right)}\left[\bar{u}_{k\left(T_{N_{t}+1}\right)}\left[a_{k\left(T_{N_{t}+1}\right)}\left(T_{N_{t}+1}\right)\right]-q\left(T_{N_{t}+1}\right) a_{k\left(T_{N_{t}+1}\right)}\left(T_{N_{t}+1}\right)\right]\right\} \\
& +\mathbb{E}_{k(t)}\left[e^{-r\left(T_{N_{t}+1}-t\right)} K_{k\left(T_{N_{t}+1}\right)}\left(T_{N_{t}+1}\right)\right] .
\end{aligned}
$$

Proof. First, notice that for all integers $n \geq 0$, we have $N_{s}=N_{t}+n$ if $s=T_{N_{t}+n}$, so the definition of $K_{k(t)}(t)$ implies

$$
\begin{gathered}
K_{k\left(T_{N_{t}+1}\right)}\left(T_{N_{t}+1}\right)=\frac{1}{r+\kappa} \mathbb{E}_{k\left(T_{N_{t}+1}\right)}\left\{\sum_{n=2}^{\infty} e^{-r\left(T_{N_{t}+n}-T_{N_{t}+1}\right)} \bar{u}_{k\left(T_{N_{t}+n}\right)}\left[a_{k\left(T_{N_{t}+n}\right)}\left(T_{N_{t}+n}\right)\right]\right\} \\
-\frac{1}{r+\kappa} \mathbb{E}_{k\left(T_{N_{t}+1}\right)}\left\{\sum_{n=2}^{\infty} e^{-r\left(T_{N_{t}+n}-T_{N_{t}+1}\right)} q\left(T_{N_{t}+n}\right) a_{k\left(T_{N_{t}+n}\right)}\left(T_{N_{t}+n}\right)\right\} .
\end{gathered}
$$

Also from the definition of $K_{k(t)}(t)$,

$$
\begin{aligned}
& K_{k(t)}(t)=\frac{1}{r+\kappa} \mathbb{E}_{k(t)}\left\{e^{-r\left(T_{N_{t}+1}-t\right)}\left[\bar{u}_{k\left(T_{N_{t}+1}\right)}\left[a_{k\left(T_{N_{t}+1}\right)}\left(T_{N_{t}+1}\right)\right]-q\left(T_{N_{t}+1}\right) a_{k\left(T_{N_{t}+1}\right)}\left(T_{N_{t}+1}\right)\right]\right\} \\
& +\frac{1}{r+\kappa} \mathbb{E}_{k(t)}\left\{\sum_{n=2}^{\infty} e^{-r\left(T_{N_{t}+n}-t\right)}\left[\bar{u}_{k\left(T_{N_{t}+n}\right)}\left[a_{k\left(T_{N_{t}+n}\right)}\left(T_{N_{t}+n}\right)\right]-q\left(T_{N_{t}+n}\right) a_{k\left(T_{N_{t}+n}\right)}\left(T_{N_{t}+n}\right)\right]\right\} \\
& =\frac{1}{r+\kappa} \mathbb{E}_{k(t)}\left\{e^{-r\left(T_{N_{t}+1}-t\right)}\left[\bar{u}_{k\left(T_{N_{t}+1}\right)}\left[a_{k\left(T_{N_{t}+1}\right)}\left(T_{N_{t}+1}\right)\right]-q\left(T_{N_{t}+1}\right) a_{k\left(T_{N_{t}+1}\right)}\left(T_{N_{t}+1}\right)\right]\right\} \\
& +\mathbb{E}_{k(t)} e^{-r\left(T_{N_{t}+1}-t\right)}\left[\frac{1}{r+\kappa} \mathbb{E}_{k\left(T_{N_{t}+1}\right)}\left\{\sum_{n=2}^{\infty} e^{-r\left(T_{N_{t}+n}-T_{N_{t}+1}\right)} \bar{u}_{k\left(T_{N_{t}+n}\right)}\left[a_{k\left(T_{N_{t}+n}\right)}\left(T_{N_{t}+n}\right)\right]\right\}\right] \\
& -\mathbb{E}_{k(t)} e^{-r\left(T_{N_{t}+1}-t\right)}\left[\frac{1}{r+\kappa} \mathbb{E}_{k\left(T_{N_{t}+1}\right)}\left\{\sum_{n=2}^{\infty} e^{-r\left(T_{N_{t}+n}-T_{N_{t}+1}\right)} q\left(T_{N_{t}+n}\right) a_{k\left(T_{N_{t}+n}\right)}\left(T_{N_{t}+n}\right)\right\}\right] \\
& =\frac{1}{r+\kappa} \mathbb{E}_{k(t)}\left\{e^{-r\left(T_{N_{t}+1}-t\right)}\left[\bar{u}_{k\left(T_{N_{t}+1}\right)}\left[a_{k\left(T_{N_{t}+1}\right)}\left(T_{N_{t}+1}\right)\right]-q\left(T_{N_{t}+1}\right) a_{k\left(T_{N_{t}+1}\right)}\left(T_{N_{t}+1}\right)\right]\right\} \\
& +\mathbb{E}_{k(t)}\left[e^{-r\left(T_{N_{t}+1}-t\right)} K_{k\left(T_{N_{t}+1}\right)}\left(T_{N_{t}+1}\right)\right] .
\end{aligned}
$$

The last equality follows from (75).

Lemma 8 Consider an investor who, at some initial time $t \geq 0$, starts with asset position a and preference type $k(t) \in \mathbb{X}$, and suppose that there exists a number $B>\max _{j} \bar{u}_{j}^{\prime}(\infty)$ such that $q(s) \geq B$ for all $s \geq t$.

(i) The maximum value of (71), i.e., $V_{k(t)}^{*}(a, t)$, satisfies the functional equation (27).

(ii) The asset plan that solves (71), i.e., $\left(a_{k\left(T_{N_{s}}\right)}(s), s \in[t, \infty)\right)$, satisfies

$$
V_{k(t)}^{*}\left[a_{k\left(T_{N_{t}}\right)}\left(T_{N_{t}}\right), t\right]=\frac{\bar{u}_{k(t)}\left[a_{k\left(T_{N_{t}}\right)}\left(T_{N_{t}}\right)\right]}{r+\kappa}+\mathbb{E}_{k(t)}\left[e^{-r\left(T_{N_{t}+1}-t\right)} p\left(T_{N_{t}+1}\right) a_{k\left(T_{N_{t}}\right)}\left(T_{N_{t}}\right)\right]
$$


$+\mathbb{E}_{k(t)}\left[e^{-r\left(T_{N_{t}+1}-t\right)}\left\{V_{k\left(T_{N_{t}+1}\right)}^{*}\left[a_{k\left(T_{N_{t}+1}\right)}\left(T_{N_{t}+1}\right), T_{N_{t}+1}\right]-p\left(T_{N_{t}+1}\right) a_{k\left(T_{N_{t}+1}\right)}\left(T_{N_{t}+1}\right)\right\}\right]$.

(iii) Let $\left(a_{k\left(T_{N_{s}}\right)}(s), s \in[t, \infty)\right)$ be the asset plan induced by (27), i.e., the asset plan in (6), and with

$$
\lim _{n \rightarrow \infty} \mathbb{E}_{i}\left[e^{-r\left(T_{N_{t}+n}-t\right)} p\left(T_{N_{t}+n}\right) a_{k\left(T_{N_{t}+n}\right)}\left(T_{N_{t}+n}\right)\right]=0
$$

for each $i \in \mathbb{X}$. Then this asset plan achieves the maximum in (71).

(iv) Let $\left(a_{k\left(T_{N_{s}}\right)}(s), s \in[t, \infty)\right)$ be the asset plan induced by (27), and assume it satisfies (76). If $V_{i}(a, t)$ solves (27) and satisfies

$$
\lim _{n \rightarrow \infty} \mathbb{E}_{i}\left[e^{-r\left(T_{N_{t}+n}-t\right)} V_{k\left(T_{N_{t}+n}\right)}\left[a_{k\left(T_{N_{t}+n}\right)}\left(T_{N_{t}+n}\right), T_{N_{t}+n}\right]\right]=0
$$

for each $i \in \mathbb{X}$, then $V_{i}(a, t)=V_{i}^{*}(a, t)$.

Proof. ( $i)$ If we let $V^{*}(a, t) \equiv\left\{V_{i}^{*}(a, t)\right\}_{i=1}^{I}$, and regard the right side of (27) as a map $F$, we need to show $F V^{*}=V^{*}$. Substitute $V^{*}(a, t)$ as given by (74), into (27):

$$
\begin{aligned}
\left(F V^{*}\right)(a, t, i) & =\frac{\bar{u}_{i}(a)}{r+\kappa}+\mathbb{E}_{i}\left[e^{-r\left(T_{N_{t}+1}-t\right)}\left\{p\left(T_{N_{t}+1}\right) a+\max _{a^{\prime} \geq 0}\left[V_{k\left(T_{N_{t}+1}\right)}^{*}\left(a^{\prime}, T_{N_{t}+1}\right)-p\left(T_{N_{t}+1}\right) a^{\prime}\right]\right\}\right] \\
& =\frac{\bar{u}_{i}(a)}{r+\kappa}+\left[p(t)-\frac{q(t)}{r+\kappa}\right] a+\mathbb{E}_{i}\left[e^{-r\left(T_{N_{t}+1}-t\right)} K_{k\left(T_{N_{t}+1}\right)}\left(T_{N_{t}+1}\right)\right] \\
& +\frac{1}{r+\kappa} \mathbb{E}_{i}\left\{e^{-r\left(T_{N_{t}+1}-t\right)}\left[\bar{u}_{k\left(T_{N_{t}+1}\right)}\left[a_{k\left(T_{N_{t}+1}\right)}\left(T_{N_{t}+1}\right)\right]-q\left(T_{N_{t}+1}\right) a_{k\left(T_{N_{t}+1}\right)}\left(T_{N_{t}+1}\right)\right]\right\} \\
& =\frac{\bar{u}_{i}(a)}{r+\kappa}+\left[p(t)-\frac{q(t)}{r+\kappa}\right] a+K_{i}(t) \\
& =V_{i}^{*}(a, t),
\end{aligned}
$$

where the third equality follows from Lemma 7.

(ii) From (74),

$$
V_{k(t)}^{*}\left[a_{k\left(T_{N_{t}}\right)}\left(T_{N_{t}}\right), t\right]=\frac{\bar{u}_{k(t)}\left[a_{k\left(T_{N_{t}}\right)}\left(T_{N_{t}}\right)\right]}{r+\kappa}+\left[p(t)-\frac{q(t)}{r+\kappa}\right] a_{k\left(T_{N_{t}}\right)}\left(T_{N_{t}}\right)+K_{k(t)}(t)
$$




$$
\begin{aligned}
& =\frac{\bar{u}_{k(t)}\left[a_{k\left(T_{N_{t}}\right)}\left(T_{N_{t}}\right)\right]}{r+\kappa}+\mathbb{E}_{k(t)}\left[e^{-r\left(T_{N_{t}+1}-t\right)} p\left(T_{N_{t}+1}\right) a_{k\left(T_{N_{t}}\right)}\left(T_{N_{t}}\right)\right]+K_{k(t)}(t) \\
& =\frac{\bar{u}_{k(t)}\left[a_{k\left(T_{N_{t}}\right)}\left(T_{N_{t}}\right)\right]}{r+\kappa}+\mathbb{E}_{k(t)}\left[e^{-r\left(T_{N_{t}+1}-t\right)} p\left(T_{N_{t}+1}\right) a_{k\left(T_{N_{t}}\right)}\left(T_{N_{t}}\right)\right] \\
& +\frac{1}{r+\kappa} \mathbb{E}_{k(t)}\left\{e^{-r\left(T_{N_{t}+1}-t\right)}\left[\bar{u}_{k\left(T_{N_{t}+1}\right)}\left[a_{k\left(T_{N_{t}+1}\right)}\left(T_{N_{t}+1}\right)\right]-q\left(T_{N_{t}+1}\right) a_{k\left(T_{N_{t}+1}\right)}\left(T_{N_{t}+1}\right)\right]\right\} \\
& +\mathbb{E}_{k(t)}\left[e^{-r\left(T_{N_{t}+1}-t\right)} K_{k\left(T_{N_{t}+1}\right)}\left(T_{N_{t}+1}\right)\right] \\
& =\frac{\bar{u}_{k(t)}\left[a_{k\left(T_{N_{t}}\right)}\left(T_{N_{t}}\right)\right]}{r+\kappa}+\mathbb{E}_{k(t)}\left[e^{-r\left(T_{N_{t}+1}-t\right)} p\left(T_{N_{t}+1}\right) a_{k\left(T_{N_{t}}\right)}\left(T_{N_{t}}\right)\right] \\
& +\mathbb{E}_{k(t)}\left[e^{-r\left(T_{N_{t}+1}-t\right)}\left\{V_{k\left(T_{N_{t}+1}\right)}^{*}\left[a_{k\left(T_{N_{t}+1}\right)}\left(T_{N_{t}+1}\right), T_{N_{t}+1}\right]-p\left(T_{N_{t}+1}\right) a_{k\left(T_{N_{t}+1}\right)}\left(T_{N_{t}+1}\right)\right\}\right] .
\end{aligned}
$$

The second equality follows from the definition of $q(t)$, the third equality from Lemma 7 , and the fourth equality from the fact that

$$
\begin{aligned}
& V_{k\left(T_{N_{t}+1}\right)}^{*}\left[a_{k\left(T_{N_{t}+1}\right)}\left(T_{N_{t}+1}\right), T_{N_{t}+1}\right]=\frac{\bar{u}_{k\left(T_{N_{t}+1}\right)}\left[a_{k\left(T_{N_{t}+1}\right)}\left(T_{N_{t}+1}\right)\right]}{r+\kappa} \\
& +\left[p\left(T_{N_{t}+1}\right)-\frac{q\left(T_{N_{t}+1}\right)}{r+\kappa}\right] a_{k\left(T_{N_{t}+1}\right)}\left(T_{N_{t}+1}\right)+K_{k\left(T_{N_{t}+1}\right)}\left(T_{N_{t}+1}\right) .
\end{aligned}
$$

(iii) Immediate from Proposition 12.

(iv) By (3) and (6), we can write (27) as

$$
\begin{aligned}
V_{k(t)}(a, t) & =\frac{\bar{u}_{k(t)}(a)}{r+\kappa}+\mathbb{E}_{k(t)}\left\{e^{-r\left(T_{N_{t}+1}-t\right)} p\left(T_{N_{t}+1}\right)\left[a-a_{k\left(T_{N_{t}+1}\right)}\left(T_{N_{t}+1}\right)\right]\right\} \\
& +\mathbb{E}_{k(t)}\left\{e^{-r\left(T_{N_{t}+1}-t\right)} V_{k\left(T_{N_{t}+1}\right)}\left[a_{k\left(T_{N_{t}+1}\right)}\left(T_{N_{t}+1}\right), T_{N_{t}+1}\right]\right\} .
\end{aligned}
$$

Iterate this expression forward $M-1$ times (using the Law of Iterated Expectations and (69)) to arrive at

$$
\begin{aligned}
& V_{k(t)}^{M}(a, t)=\frac{\bar{u}_{k(t)}(a)}{r+\kappa}+\left[p(t)-\frac{q(t)}{r+\kappa}\right] a-\mathbb{E}_{k(t)}\left[e^{-r\left(T_{N_{t}+M}-t\right)} p\left(T_{N_{t}+M}\right) a_{k\left(T_{N_{t}+M}\right)}\left(T_{N_{t}+M}\right)\right] \\
& +\frac{1}{r+\kappa} \mathbb{E}_{k(t)}\left\{\sum_{n=1}^{M-1} e^{-r\left(T_{N_{t}+n}-t\right)}\left[\bar{u}_{k\left(T_{N_{t}+n}\right)}\left[a_{k\left(T_{N_{t}+n}\right)}\left(T_{N_{t}+n}\right)\right]-q\left(T_{N_{t}+n}\right) a_{k\left(T_{N_{t}+n}\right)}\left(T_{N_{t}+n}\right)\right]\right\} \\
& +\mathbb{E}_{k(t)}\left[e^{-r\left(T_{N_{t}+M}-t\right)} V_{k\left(T_{N_{t}+M}\right)}\left[a_{k\left(T_{N_{t}+M}\right)}\left(T_{N_{t}+M}\right), T_{N_{t}+M}\right]\right] .
\end{aligned}
$$

A function $V_{k(t)}(a, t)$ that solves (27) must satisfy (78) for all $M$, so the solution is $V_{k(t)}(a, t)=$ $\lim _{M \rightarrow \infty} V_{k(t)}^{M}(a, t)$, provided this limit exists. From (78),

$$
\lim _{M \rightarrow \infty} V_{k(t)}^{M}(a, t)=\frac{\bar{u}_{k(t)}(a)}{r+\kappa}+\left[p(t)-\frac{q(t)}{r+\kappa}\right] a-\lim _{M \rightarrow \infty} \mathbb{E}_{k(t)}\left[e^{-r\left(T_{N_{t}+M}-t\right)} p\left(T_{N_{t}+M}\right) a_{k\left(T_{N_{t}+M}\right)}\left(T_{N_{t}+M}\right)\right]
$$




$$
\begin{aligned}
& +\lim _{M \rightarrow \infty} \mathbb{E}_{k(t)}\left\{\sum_{n=1}^{M-1} e^{-r\left(T_{N_{t}+n}-t\right)}\left[\frac{\bar{u}_{k\left(T_{N_{t}+n}\right)}\left[a_{k\left(T_{N_{t}+n}\right)}\left(T_{N_{t}+n}\right)\right]}{r+\kappa}-\frac{q\left(T_{N_{t}+n}\right)}{r+\kappa} a_{k\left(T_{N_{t}+n}\right)}\left(T_{N_{t}+n}\right)\right]\right\} \\
& +\lim _{M \rightarrow \infty} \mathbb{E}_{k(t)}\left[e^{-r\left(T_{N_{t}+M}-t\right)} V_{k\left(T_{N_{t}+M}\right)}\left[a_{k\left(T_{N_{t}+M}\right)}\left(T_{N_{t}+M}\right), T_{N_{t}+M}\right]\right] \\
& =\frac{\bar{u}_{k(t)}(a)}{r+\kappa}+\left[p(t)-\frac{q(t)}{r+\kappa}\right] a \\
& +\lim _{M \rightarrow \infty} \mathbb{E}_{k(t)}\left\{\sum_{n=1}^{M-1} e^{\left.-r\left(T_{N_{t}+n}-t\right)\left[\frac{\bar{u}_{k\left(T_{N_{t}+n}\right)}\left[a_{k\left(T_{N_{t}+n}\right)}\left(T_{N_{t}+n}\right)\right]}{r+\kappa}-\frac{q\left(T_{N_{t}+n}\right)}{r+\kappa} a_{k\left(T_{N_{t}+n}\right)}\left(T_{N_{t}+n}\right)\right]\right\} .}\right.
\end{aligned}
$$

The second equality follows from (76) and (77). Since $\left\{a_{k\left(T_{N_{t}+n}\right)}\left(T_{N_{t}+n}\right)\right\}_{n=1}^{\infty}$ is bounded and $\bar{u}_{i}$ is continuous for every $i,\left\{\bar{u}_{k\left(T_{N_{t}+n}\right)}\left[a_{k\left(T_{N_{t}+n}\right)}\left(T_{N_{t}+n}\right)\right]\right\}_{n=1}^{\infty}$ is bounded above. Hence, without loss of generality, we can rescale $u_{i}$ for each $i$ so that $\left\{-\bar{u}_{k\left(T_{N_{t}+n}\right)}\left[a_{k\left(T_{N_{t}+n}\right)}\left(T_{N_{t}+n}\right)\right]\right\}_{n=1}^{\infty}$ is a nonnegative sequence. Then the sequence $\left\{\bar{f}_{M}\right\}_{M=1}^{\infty}$, where

$$
\bar{f}_{M}(\omega) \equiv \sum_{n=1}^{M-1} e^{-r\left(T_{N_{t}+n}-t\right)}\left[\bar{u}_{k\left(T_{N_{t}+n}\right)}\left[a_{k\left(T_{N_{t}+n}\right)}\left(T_{N_{t}+n}\right)\right]-q\left(T_{N_{t}+n}\right) a_{k\left(T_{N_{t}+n}\right)}\left(T_{N_{t}+n}\right)\right],
$$

is a nonincreasing sequence, and hence it has a limit, $\lim _{M \rightarrow \infty} \bar{f}_{M}$, which could be $-\infty$. Since $\left\{-\bar{f}_{M}\right\}_{M=1}^{\infty}$ is a monotone increasing sequence of measurable functions that converge pointwise to $-\lim _{M \rightarrow \infty} \bar{f}_{M}$, by the Monotone Convergence Theorem (e.g., Theorem 7.8 in Stokey and Lucas, 1989), we have $\lim _{M \rightarrow \infty} \mathbb{E}_{k(t)}\left[\bar{f}_{M}\right]=\mathbb{E}_{k(t)}\left[\lim _{M \rightarrow \infty} \bar{f}_{M}\right]=(r+\kappa) K_{k(t)}(t)$, and therefore for every $k(t) \in \mathbb{X}$,

$$
\lim _{M \rightarrow \infty} V_{k(t)}^{M}(a, t)=\frac{\bar{u}_{k(t)}(a)}{r+\kappa}+\left[p(t)-\frac{q(t)}{r+\kappa}\right] a+K_{k(t)}(t)=V_{k(t)}^{*}(a, t) .
$$

This concludes the proof.

Lemma 8 establishes a Principle of Optimality for the economy we analyze: Part $(i)$ shows that $V_{k(t)}^{*}(a, t)$, the maximum value of the investor's problem given in (71), satisfies the functional equation (1) with asset holdings and fees given by (2) (which is equivalent to the functional equation (27)). Part (ii) establishes that the asset plan that solves (71) is an optimal plan implied by the functional equation (1) when this functional equation is evaluated at $V_{k(t)}^{*}(a, t)$. Part (iii) is a partial converse of part (ii): it proves that the asset plan that is optimal according to the functional equation (27), and that satisfies the boundedness condition (76), is the same asset plan that achieves the maximum of (71). Part $(i v)$ is a partial converse of Part $(i)$ : it shows that $V_{k(t)}^{*}(a, t)$ is the only solution of the functional equation (27) that satisfies the boundedness condition (77). 


\section{E Related literature}

In this appendix we draw connections to some related literature.

\section{E.1 Search models of over-the-counter markets}

Traders who operate in markets with OTC-style frictions will seek to mitigate these trading frictions by adjusting their asset positions so as to reduce their trading needs. Our analysis has shown that this is a critical aspect of investor behavior in illiquid markets. To illustrate this point, in this section we derive the main predictions of a version DGP's model and contrast them with those of a special case of our formulation. This comparison will underscore the fact that the type of "liquidity hedging" that we have identified - and that only becomes possible with unrestricted asset holdings - generates new insights on how trading frictions shape the various dimensions of market liquidity, alters the empirical predictions of the theory, and leads to a different assessment of their normative implications.

We will contrast the empirical predictions of DGP's model with those of a special case of our model with $\mathbb{X}=\{1,2\}$ and $u_{i}(a)=\varepsilon_{i} \frac{a^{1-\sigma}}{1-\sigma}$ for $i \in \mathbb{X}$ and $\sigma>0$. We focus on the version of DGP's model with no inter-investor meetings (e.g., the version that DGP use in their Theorem 4 and part $(i)$ of Theorem 6). DGP restrict $a \in\{0,1\}$ and let $u_{i j}$ denote the flow utility of an investor with asset position $i \in\{0,1\}$ and preference type $j \in\{0,1\}$. $^{35}$ DGP assume $u_{00}=u_{01}=0$, so for comparison purposes, we do the same hereafter. To simplify the notation, in both models we let $\pi$ denote the steady-state fraction of investors with high valuation. ${ }^{36}$

Price. Since asset holdings are indivisible in DGP, equilibrium in the interdealer market requires investors who are on the long side of the market to be indifferent between trading and not trading. It is easy to show that in steady state, investors who want to sell are on the short side if and only if $A<\pi$. The equilibrium price in the interdealer market is

$$
p= \begin{cases}\frac{1}{r} \frac{(r+\kappa) u_{11}+\delta \bar{u}}{r+\kappa+\delta} & \text { if } A<\pi \\ \frac{1}{r} \frac{(r+\kappa) u_{10}+\delta \bar{u}}{r+\kappa+\delta} & \text { if } \pi<A\end{cases}
$$

where $\bar{u} \equiv \pi_{1} u_{11}+\pi_{0} u_{10} \cdot{ }^{37}$

\footnotetext{
${ }^{35}$ DGP state their restriction on asset holdings as $a \in[0,1]$ but only study equilibria in which agents hold either 0 or 1 unit of the asset, which is effectively equivalent to imposing the restriction $a \in\{0,1\}$.

36 "High valuation" corresponds to the index "2" in our formulation and "1" in DGP.

${ }^{37}$ If $A=\pi, p \in\left[\frac{(r+\kappa) u_{10}+\delta \bar{u}}{r(r+\kappa+\delta)}, \frac{(r+\kappa) u_{11}+\delta \bar{u}}{r(r+\kappa+\delta)}\right]$ and the equilibrium price in the interdealer market is indeterminate.
} 
The asset holding restrictions in DGP are also the reason why the asset price in their theory is independent of the stock of assets, $A$, for any $A<\pi$ and for any $A>\pi$, with a discontinuity at $A=\pi$. In contrast, the asset price in our model is smooth and decreasing in $A$. For example, in the special case of our model that we are considering in this section, $p=\left(\sum_{i} \pi_{i} \bar{\varepsilon}_{i}^{1 / \sigma}\right)^{\sigma} / r A^{\sigma} .38$ The behavior of the asset price in response to changes in the trading frictions in DGP depends critically on the level of $A$. From (79), $p$ is increasing in $\alpha$ (decreasing in $\eta$ ) if $A<\pi$ but decreasing in $\alpha$ (increasing in $\eta$ ) if $A>\pi$. In contrast, with unrestricted asset holdings these extensive-margin considerations are irrelevant to assess the impact of trading frictions on the asset price (recall Proposition 4).

Trade volume. Trade volume is $\mathcal{V}=\alpha \frac{\delta \pi(1-\pi)}{\alpha+\delta} \frac{\left(\bar{\varepsilon}_{2}\right)^{1 / \sigma}-\left(\bar{\varepsilon}_{1}\right)^{1 / \sigma}}{\pi\left(\bar{\varepsilon}_{2}\right)^{1 / \sigma}+(1-\pi)\left(\bar{\varepsilon}_{1}\right)^{1 / \sigma}} A$ in our model and $\mathcal{V}_{D G P}=$ $\alpha \frac{\delta \pi(1-\pi)}{\alpha+\delta} \min \left\{\frac{A}{\pi}, \frac{1-A}{1-\pi}\right\}$ in DGP. The latter is independent of the dealers' bargaining power, $\eta$, and of all preference parameters and holding payoffs (e.g., $r, k$ ). In contrast, these parameters are critical determinants of trade volume in our theory, as they influence the investors' choices of asset holdings (the second factor in $\mathcal{V}$ ). Our model predicts that markets in which dealers have less market power will tend to exhibit larger trade volume. ${ }^{39}$

Transaction costs. DGP's transaction costs can be expressed in terms of the intermediation fees $\phi_{01}$ and $\phi_{10}$ that dealers charge investors who want to buy and sell, respectively. The equilibrium spread is $s=\frac{\eta\left(u_{11}-u_{10}\right)}{r+\kappa+\delta} .40$ Conditional on having contacted an investor, the expected intermediation fee that accrues to a dealer in DGP is $\Phi_{D G P}=\frac{\delta \pi(1-\pi)}{\alpha+\delta} \min \left\{\frac{A}{\pi}, \frac{1-A}{1-\pi}\right\} s$. This key determinant of dealers' incentives to make markets is decreasing in the investors' contact rate with dealers, $\alpha$, and increasing in the dealers' bargaining power, $\eta$. In contrast, as we have shown analytically in Proposition 1, in our model with no restrictions on asset holdings it is natural for the average fee to be nonmonotonic in $\alpha$ and $\eta$. Our theory suggests that these nonmonotonicities can be important. From an applied standpoint, they help explain how OTC

\footnotetext{
${ }^{38}$ Notice that we obtain DGP's formulation with $A<\pi$ as a special case of ours when $\sigma \rightarrow 0$.

${ }^{39}$ Apart from these qualitative differences, the theory with unrestricted portfolios also has different quantitative implications for the relationship between trade volume and trading frictions. For example, DGP's model has a sharp empirical implication: the elasticity of trade volume with respect to trading frictions equals $\frac{\delta}{\alpha+\delta} \in(0,1)$. In contrast, in the model with unrestricted asset holdings the corresponding elasticity is larger by an amount that equals the elasticity of $\left(a_{2}-a_{1}\right)$ with respect to $\alpha$-which is positive, capturing the notion that each investor wishes to conduct a larger trade when frictions are reduced.

${ }^{40}$ Since asset holdings in DGP are restricted to lie in $\{0,1\}$, every trade is of size 1 and hence $\phi_{01}+\phi_{10}=s$. In addition, the indivisibility assumption implies that dealers either charge a fee on asset sales or on asset purchases, but not both. Specifically, if $A<\pi$ then $\phi_{01}=0$ and investors only pay a fee $\phi_{10}=s$ when they sell. Conversely, if $\pi<A, \phi_{10}=0$ and investors only pay a fee $\phi_{01}=s$ when they buy.
} 
markets have reacted to recent changes in their market structure (see Lagos and Rocheteau, 2006). From a theoretical standpoint, they can generate self-fulfilling liquidity shortages in markets with free entry of dealers (Section 6). ${ }^{41}$

Another key difference with DGP is the fact that since the equilibrium in the model with unrestricted portfolios implies a nondegenerate distribution of trade sizes, our theory has predictions for the relationship between transaction costs and transaction sizes. As we showed in Lemma 4, transaction costs are increasing in the size of the transaction. Thus, if $a_{i}-a_{j}>a_{i}-a_{k}>0$, then the effective price at which the investor buys is $\hat{p}_{j i}>\hat{p}_{k i}$, i.e., he effectively pays higher prices when he conducts larger purchases. Conversely, $\hat{p}_{j i}<\hat{p}_{k i}$ if $a_{i}-a_{j}<a_{i}-a_{k}<0$, i.e., he effectively receives lower prices when he conducts larger sales. In other words, the theory with unrestricted asset holdings naturally generates instances of price concession, which are commonplace in OTC markets. ${ }^{42}$

Execution delays. DGP endogenized trading delays by allowing a single monopolist dealer to choose search intensity once-and-for-all at the beginning of time. Free entry of competing dealers or market-makers is a feature of most OTC markets; however, the implications of this microstructure have not yet been explored in the literature. We find that allowing for free entry of dealers is a natural way to endogenize execution delays and the amount of liquidity supplied by dealers, and that it provides an important channel through which changes in market conditions affect transaction costs and trade volume. In addition, the interaction between free entry and unrestricted asset holdings leads to a natural kind of strategic complementarity that can help rationalize self-fulfilling liquidity shortages in markets with OTC-style frictions (Section 6).

Welfare. The equilibrium allocation is always constrained efficient in the baseline model of DGP - regardless of the value of $\eta$ - which stands in contrast to the finding we report in Proposition 2. The reason is that in our model investors choose asset holdings, while this intensive margin is absent in DGP. For the same reason, the inefficiency result we find in the context of the model with free entry also has no counterpart in DGP.

\footnotetext{
${ }^{41}$ The spread, $s$, is decreasing in $\alpha$ and increasing in $\eta$ in this version of DGP with no inter-investor meetings. One can also verify that the average effective spread weighted by the sizes of each trade and expressed as a proportion of the price is also decreasing in $\alpha$ and increasing in $\eta$. The behavior of this measure of the marketwide spread, i.e., (38) in Lagos and Rocheteau (2006), is much more complicated in our model, where the investors' expected holding payoffs, their individual asset demands, the asset price, and the whole distribution of asset holdings change in response to a change in $\alpha$. Our numerical work, some of which we have reported in Lagos and Rocheteau (2006), is in accordance with the predictions of DGP.

${ }^{42}$ See Section 4.3 in Harris (2003).
} 
A paper that is closely related to ours is an independent contribution by Gârleanu (2006), which studies the asset pricing and volume implications of infrequent (Poisson) trading opportunities. Some of our findings are similar: he also finds that under certain conditions (e.g., a mean-reversion property of preference shocks), investors take more extreme positions when trading delays are short. Also, Gârleanu stresses that the asset price is not affected by the trading frictions - which is true in our model for a particular specification of the utility function (Proposition 4). In terms of differences, trades in Gârleanu (2006) are not intermediated by dealers, so he could not consider the implications of execution delays for transaction costs and dealers' incentives to provide liquidity, which are at the center of our analysis. Also, Gârleanu (2006) formalizes the investors' motive for holding the asset by developing the "hedging needs" motive we mentioned in footnote 4 . Despite the differences in the formulations, some of our results on the effects of $\alpha$ on trade volume are remarkably similar. ${ }^{43}$

\section{E.2 Search models of money}

Here we discuss the relationship between our theory and the search-theoretic literature on monetary exchange. In contrast to the monetary literature, our model does not have fiat money as an asset, and it does not aim to explain the use or emergence of a medium of exchange. However, it shares a common objective with modern monetary theory, which is to endogenize some relevant dimensions of "liquidity." We organize the comparison around four types of results.

Endogenous distribution of asset holdings. Because of idiosyncratic (trading) shocks, under incomplete markets, our model generates a nondegenerate distribution of wealth as Green and Zhou (2002) and Molico (2006), but also Aiyagari (1994). The trading mechanism in our model is closer to the one in Molico: the asset is traded in bilateral matches, and the transaction price is determined through bargaining. In terms of the methodology, both Aiyagari (1994) and Molico (2006) solve their models numerically. Green and Zhou (2002) is closer to our analysis in that they can characterize the equilibrium and its distribution of money holdings analytically. Moreover, like us, they do not restrict their analysis to stationary equilibria. The pricing mechanism is different (Green and Zhou consider a double auction).

Bargaining and the distribution of prices. A key insight of our model is that the interme-

\footnotetext{
${ }^{43}$ See the discussion around Proposition 9 in Appendix B for details.
} 
diation fee depends on the (endogenous) asset position of the investor. Similarly, in monetary search models with bargaining, the transaction price depends on the traders' money balances. This dependence occurs through (at least) two channels. First, the buyer can be constrained by his money balances. This mechanism is present even in models with a degenerate distribution of money balances, such as Shi (1997) and Lagos and Wright (2005). Second, the money holdings of an agent affect his marginal utility of wealth, and hence the terms of trade. These two effects are absent from our model, since our investors never face binding borrowing constraints, and the marginal utility of wealth is normalized to one due to the quasi-linear preferences. An investor's asset holdings influence the outcome of the bargaining in our model because this asset position determines the size of the gains from trade that will be generated for readjusting the investor's asset holdings.

Uniqueness of the equilibrium. The equilibrium (not just the steady state) is unique in our model. In contrast, the model of fiat money of Green and Zhou can display multiple equilibria. This indeterminacy is a general feature of models of fiat money. Even in models with a degenerate distribution of money balances, e.g., Lagos and Wright (2005), the equilibrium is typically not unique, unless one restricts attention to steady-state monetary equilibria. Models of monetary exchange consider environments where the asset being traded is fiat money, whose value emerges endogenously when it is valued as a medium of exchange that mitigates a doublecoincidence of wants problem. In contrast, in our model and the rest of the literature that deals with the trading process in OTC markets, the asset being traded is not used to facilitate trades; it is valued for its intrinsic characteristics (e.g., dividend flow).

Endogenous trading delays and multiple equilibria. In our model, the multiplicity of steady-state equilibria with dealer entry arises from complementarities between investors' asset demands and dealers' entry decision. If more dealers participate in the market, it is easier for investors to readjust their asset holdings, which induces them to take more extreme positions, and this in turn makes it profitable for dealers to enter. Rocheteau and Wright (2005) consider a monetary search model with free entry of sellers and find that the strategic complementarities between the sellers' entry decision and the buyers' demand for real balances generate multiple steady-state equilibria. If buyers accumulate more real balances, the buyer and the seller are able to exploit larger gains from trade, which gives more incentives for sellers to participate in the market. In both models, the multiplicity does not require increasing returns to scale in the 
matching function as in Diamond (1982) or as in most recent search models of financial markets, e.g., Vayanos and Weill (2008). A key difference between our model and Rocheteau and Wright (2005) is the opportunity cost from holding real balances in the latter, which has no counterpart in our formulation. If the opportunity cost from holding cash balances to make a purchase is zero (e.g., if the nominal interest rate is zero), then the multiplicity of (active) steady-state equilibria in that model disappears. In contrast, the multiplicity in our model obtains even though investors do not bear any opportunity cost (e.g., forgone interest) while searching for an asset to purchase (since they have access to a technology to produce the numéraire good). Also, notice that the gains from trade in Rocheteau and Wright (2005) depend on the mean of the distribution of real balances (since the distribution of real balances is degenerate as in Lagos and Wright, 2005), which is independent of trading frictions when the nominal interest rate is zero. In our model it is the second moment, which is endogenous and depends on the trading frictions, what gives rise to multiple steady-state equilibria. 


\section{References}

[1] Aiyagari, S. R. (1994): "Uninsured Idiosyncratic Risk and Aggregate Saving," Quarterly Journal of Economics, 109, 659-84.

[2] Binmore, K. (1987): "Perfect Equilibria in Bargaining Models," in The Economics of Bargaining, edited by K. Binmore and P. Dasgupta. Oxford: Basil Blackwell.

[3] Binmore, K., A. Rubinstein, and A. Wolinsky (1986): "The Nash Bargaining Solution in Economic Modelling," RAND Journal of Economics, 17, 176-88.

[4] Burdett, K., A. Trejos, and R. Wright (2001): "Cigarette Money," Journal of Economic Theory, 99, 117-42.

[5] Burnside, C., M. Eichenbaum, I. Kleshchelski, and S. Rebelo (2006): "The Returns to Currency Speculation," unpublished manuscript.

[6] Constantinides, G. (1986): "Capital Market Equilibrium with Transaction Costs," Journal of Political Economy, 94, 842-62.

[7] Diamond, P. A. (1982): "Aggregate Demand Management in Search Equilibrium," Journal of Political Economy, 90, 881-94.

[8] Duffie, D., N. Gârleanu, and L. H. Pedersen (2005): "Over-the-Counter Markets," Econometrica, $73,1815-47$.

[9] Duffie, D., N. Gârleanu, and L. H. Pedersen (2007): "Valuation in Over-the-Counter Markets," Review of Financial Studies, 20, 1865-1900.

[10] Duffie, D., and Y. Sun (2007): "Existence of Independent Random Matching," Annals of Applied Probability, 17, 386-419.

[11] Gârleanu, N. (2006): "Portfolio Choice and Pricing in Illiquid Markets," unpublished manuscript.

[12] Green, E., and R. Zhou (2002): "Dynamic Monetary Equilibrium in a Random Matching Economy," Econometrica, 70, 929-69. 
[13] Green, R., B. Hollifield, and N. Schurhoff (2007): "Financial Intermediation and the Costs of Trading in an Opaque Market," Review of Financial Studies, 20, 275-314.

[14] Harris, L. (2003): Trading and Exchanges: Market Microstructure for Practitioners. New York: Oxford University Press.

[15] Harris L., and M. Piwowar (2006): "Secondary Trading Costs in the Municipal Bond Market," Journal of Finance, 61, 1361-97.

[16] Heaton, J., and D. J. Lucas (1995): "The Importance of Investor Heterogeneity and Financial Market Imperfections for the Behavior of Asset Prices," Carnegie-Rochester Conference Series on Public Policy, 42, 1-32.

[17] Hosios, A. J. (1990): "On the Efficiency of Matching and Related Models of Search and Unemployment," Review of Economic Studies, 57, 279-98.

[18] Kiyotaki, N., and R. Lagos (2007): "A Model of Job and Worker Flows," Journal of Political Economy, 115, 770-819.

[19] Kiyotaki, N., and R. Wright (1993): "A Search-Theoretic Approach to Monetary Economics," American Economic Review, 83, 63-77.

[20] Lagos, R., and G. Rocheteau (2006): "Search in Asset Markets," Federal Reserve Bank of Cleveland Working Paper 06-07.

[21] Lagos, R., and G. Rocheteau (2008): "Liquidity in Asset Markets with Search Frictions," working paper.

[22] Lagos, R., G. Rocheteau, and P. O. Weill (2007): "Crashes and Recoveries in Illiquid Markets," unpublished manuscript.

[23] Lagos, R., and R. Wright (2005): "A Unified Framework for Monetary Theory and Policy Analysis," Journal of Political Economy, 113, 463-84.

[24] Lo, A. W., H. Mamaysky, and J. Wang (2004): "Asset Prices and Trading Volume under Fixed Transactions Costs," Journal of Political Economy, 112, 1054-90.

[25] Merlo, A., and C. Wilson (1995): "Stochastic Model of Sequential Bargaining with Complete Information," Econometrica, 63, 371-99. 
[26] Merlo, A., and C. Wilson (1998): "Efficient Delays in a Stochastic Model of Bargaining," Economic Theory, 11, 9-55.

[27] Miao, J. (2006): "A Search Model of Centralized and Decentralized Trade," Review of Economic Dynamics, 9, 68-92.

[28] Molico, M. (2006): "The Distribution of Money and Prices in Search Equilibrium," International Economic Review, 47, 701-22.

[29] Osborne, M., A. Rubinstein (1994): A Course in Game Theory. Cambridge: MIT Press.

[30] Pissarides, C. A. (2000): Equilibrium Unemployment Theory, 2nd ed. Cambridge: MIT Press.

[31] Rocheteau, G., and R. Wright (2005): "Money in Search Equilibrium, in Competitive Equilibrium, and in Competitive Search Equilibrium," Econometrica, 73, 175-202.

[32] Rubinstein, A. (1982): "Perfect Equilibrium in a Bargaining Model," Econometrica, 50, 97-109.

[33] Rust, J., and G. Hall (2003): "Middlemen versus Market Makers: A Theory of Competitive Exchange," Journal of Political Economy, 111, 353-403.

[34] Schultz, P. (2001): "Corporate Bond Trading Costs: A Peek Behind the Curtain," Journal of Finance, 56, 677-98.

[35] Shi, S. (1997): "A Divisible Search Model of Fiat Money," Econometrica, 65, 75-102.

[36] Spulber, D. (1996): "Market Making by Price Setting Firms," Review of Economic Studies, $63,559-80$.

[37] Vayanos, D., and P. O. Weill (2008): "A Search-Based Theory of the On-the-Run Phenomenon," Journal of Finance, 63, 1351-89.

[38] Weill, P. O. (2007): "Leaning Against the Wind," Review of Economic Studies, 74, 132954. 